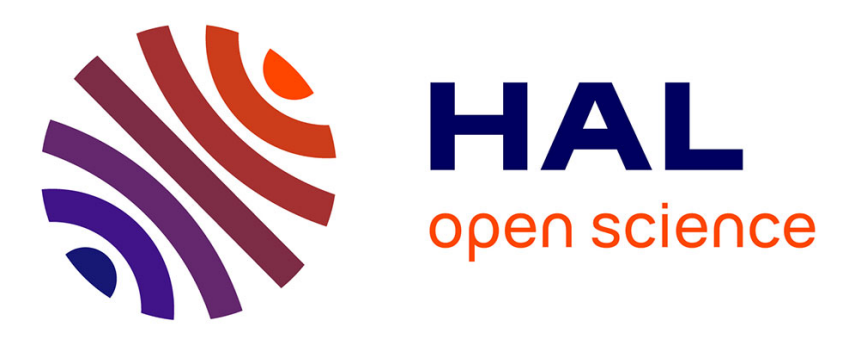

\title{
Large Eddy Simulations of gaseous flames in gas turbine combustion chambers
}

Laurent Y.M. Gicquel, Gabriel Staffelbach, Thierry Poinsot

\section{To cite this version:}

Laurent Y.M. Gicquel, Gabriel Staffelbach, Thierry Poinsot. Large Eddy Simulations of gaseous flames in gas turbine combustion chambers. Progress in Energy and Combustion Science, 2012, vol. 38, pp. 782-817. 10.1016/j.pecs.2012.04.004 . hal-00803230

\section{HAL Id: hal-00803230 \\ https://hal.science/hal-00803230}

Submitted on 21 Mar 2013

HAL is a multi-disciplinary open access archive for the deposit and dissemination of scientific research documents, whether they are published or not. The documents may come from teaching and research institutions in France or abroad, or from public or private research centers.
L'archive ouverte pluridisciplinaire HAL, est destinée au dépôt et à la diffusion de documents scientifiques de niveau recherche, publiés ou non, émanant des établissements d'enseignement et de recherche français ou étrangers, des laboratoires publics ou privés. 


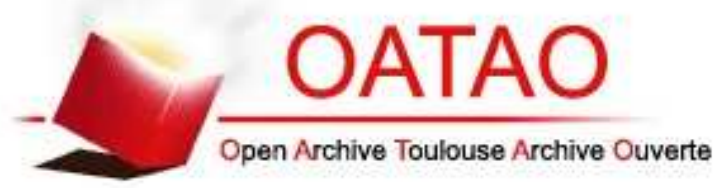

\section{Open Archive Toulouse Archive Ouverte (OATAO)}

OATAO is an open access repository that collects the work of Toulouse researchers and makes it freely available over the web where possible.

This is an author-deposited version published in: http://oatao.univ-toulouse.fr/ Eprints ID: 8578

To link to this article: DOI:10.1016/j.pecs.2012.04.004

URL : http://dx.doi.org/10.1016/j.pecs.2012.04.004

\section{To cite this version:}

Gicquel, Laurent and Staffelbach, Gabriel and Poinsot, Thierry Large Eddy Simulations of gaseous flames in gas turbine combustion chambers. (2012) Progress in Energy and Combustion Science, vol. 38 ( $\mathrm{n}^{\circ}$ 6). pp. 782-817. ISSN 0360-1285 


\title{
Large Eddy Simulations of gaseous flames in gas turbine combustion chambers
}

\author{
L.Y.M. Gicquel*, G. Staffelbach, T. Poinsot
}

CERFACS, 42 Avenue G. Coriolis, 31057 Toulouse Cedex 1, France

Keywords:

Large Eddy Simulations

Complex geometry

Swirled flows

Gaseous combustion

Turbulent combustion

Gas turbine

\begin{abstract}
A B S T R A C T
Recent developments in numerical schemes, turbulent combustion models and the regular increase of computing power allow Large Eddy Simulation (LES) to be applied to real industrial burners. In this paper, two types of LES in complex geometry combustors and of specific interest for aeronautical gas turbine burners are reviewed: (1) laboratory-scale combustors, without compressor or turbine, in which advanced measurements are possible and (2) combustion chambers of existing engines operated in realistic operating conditions. Laboratory-scale burners are designed to assess modeling and fundamental flow aspects in controlled configurations. They are necessary to gauge LES strategies and identify potential limitations. In specific circumstances, they even offer near model-free or DNS-like LES computations. LES in real engines illustrate the potential of the approach in the context of industrial burners but are more difficult to validate due to the limited set of available measurements. Usual approaches for turbulence and combustion sub-grid models including chemistry modeling are first recalled. Limiting cases and range of validity of the models are specifically recalled before a discussion on the numerical breakthrough which have allowed LES to be applied to these complex cases. Specific issues linked to real gas turbine chambers are discussed: multi-perforation, complex acoustic impedances at inlet and outlet, annular chambers... Examples are provided for mean flow predictions (velocity, temperature and species) as well as unsteady mechanisms (quenching, ignition, combustion instabilities). Finally, potential perspectives are proposed to further improve the use of LES for real gas turbine combustor designs.
\end{abstract}

Contents

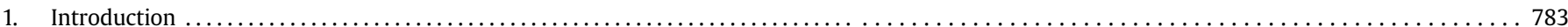

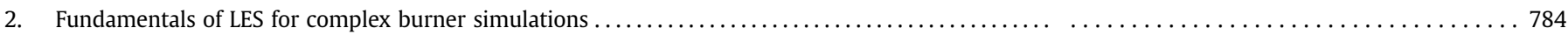

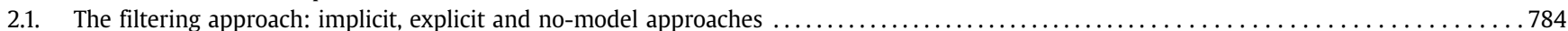

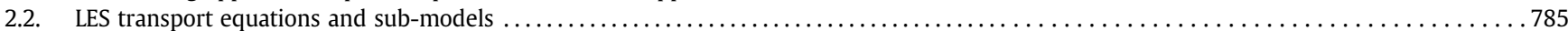

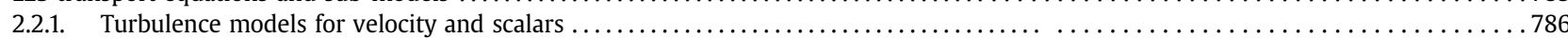

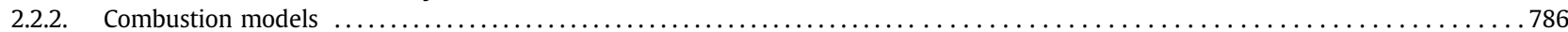

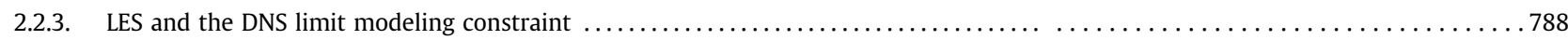

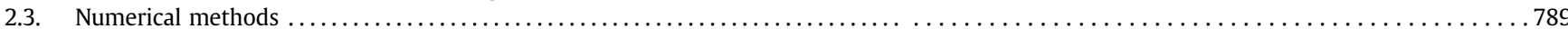

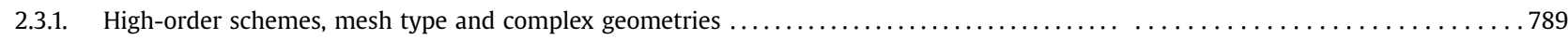

2.3.2. Implicit versus explicit time integration, incompressible, low Mach and fully compressible approaches ................790

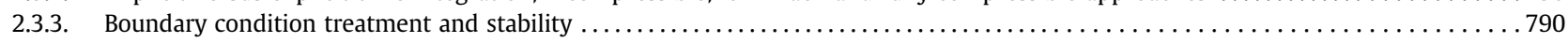

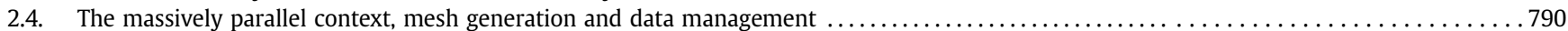

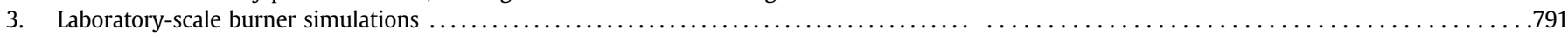

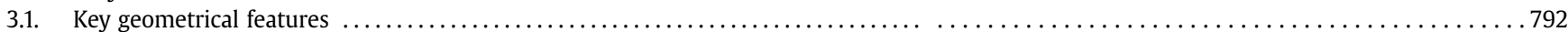

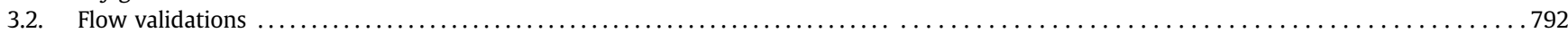

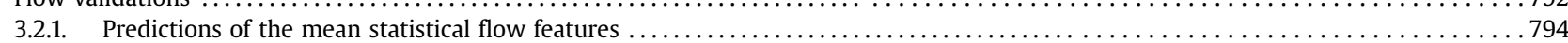

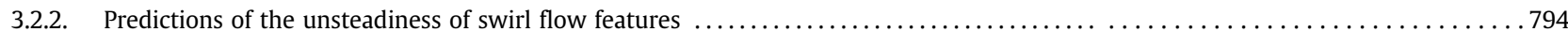

\footnotetext{
* Corresponding author. Tel.: +33 (0)5 611930 46; fax: +33 (0)5 61193000.

E-mail addresses: Laurent.Gicquel@cerfacs.fr, lgicquel@cefacs.fr (L.Y.M. Gicquel).

URL: http://www.cerfacs.fr
} 


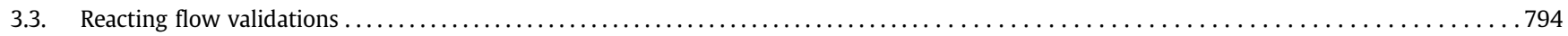

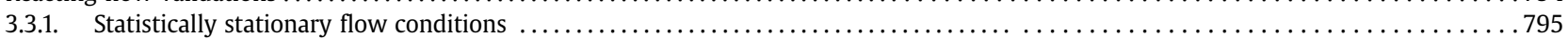

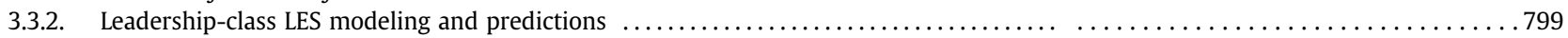

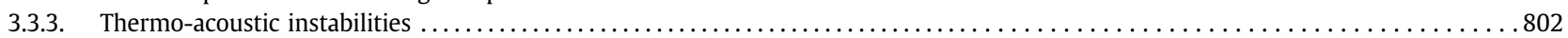

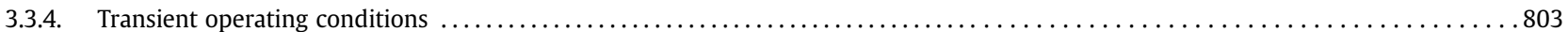

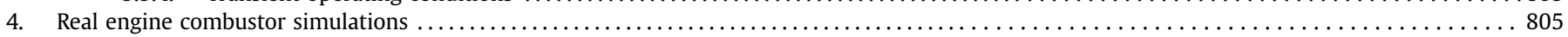

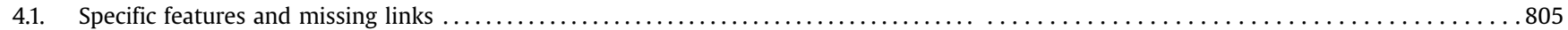

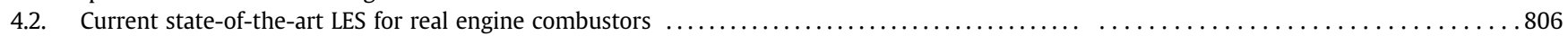

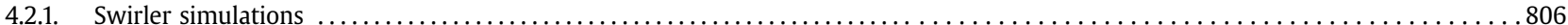

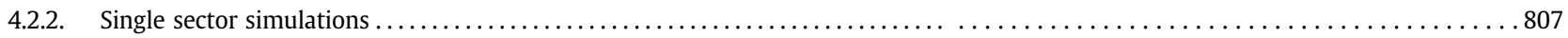

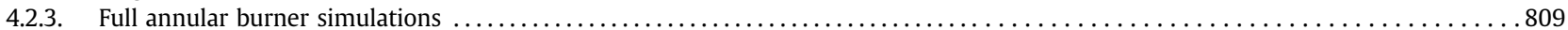

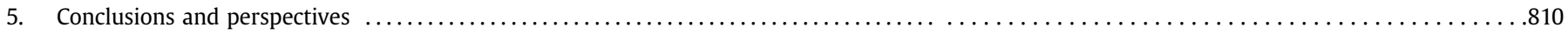

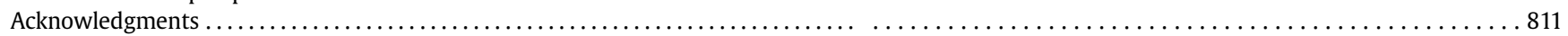

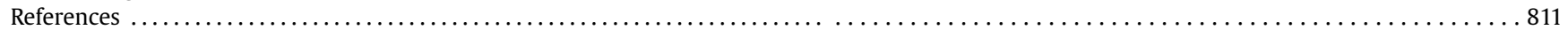

\section{Introduction}

Aeronautical turbulent reacting flows involve a wide range of scales and complexities caused by the specific shapes of engines and the combustion regimes encountered in these devices. Because of the space and weight constraints, designers need to develop burners which ensure maximum efficiency and compactness. Over the years, manufacturers have gained significant experience and existing designs largely rely on flow recirculations to increase mixing and flow-though times despite a reduced size combustion chamber. In parallel, pollutant emissions and regulations have induced changes of technology with the emergence of partially premixed and premixed burners. Multi-point fuel injection systems and staging are also being implemented as potential solutions to the new regulations. All these concepts increase the complexity of the flow and lead to specific flow dynamics and combustion responses. Although these designs are being routinely evaluated by Computational Fluid Dynamics (CFD), most present modeling strategies rely on Reynolds Average Navier-Stokes (RANS) approaches developed for mean stationary flows [1-10]. Such models benefit from extensive research and developments from the scientific community and have been successfully calibrated on simple fundamental configurations. However, the complexity of flows in modern gas turbines adds multiple constraints on RANS and limits their precision, Fig. 1. Alternative numerical solutions are thus needed to further increase the share of CFD and decrease the number of real engine tests and design iterations.

CFD alternatives to RANS for aeronautical gas turbine applications must justify the increase in development, maintenance and computer costs. These new tools need also to be compatible with existing industrial knowledge and conception rules. The use of new CFD approaches and their future in the design chain is still unclear. It will probably depend on the computing power available to engineers as well as their ability to master and analyze ever more

a RANS: Temporally/ensemble averaged field
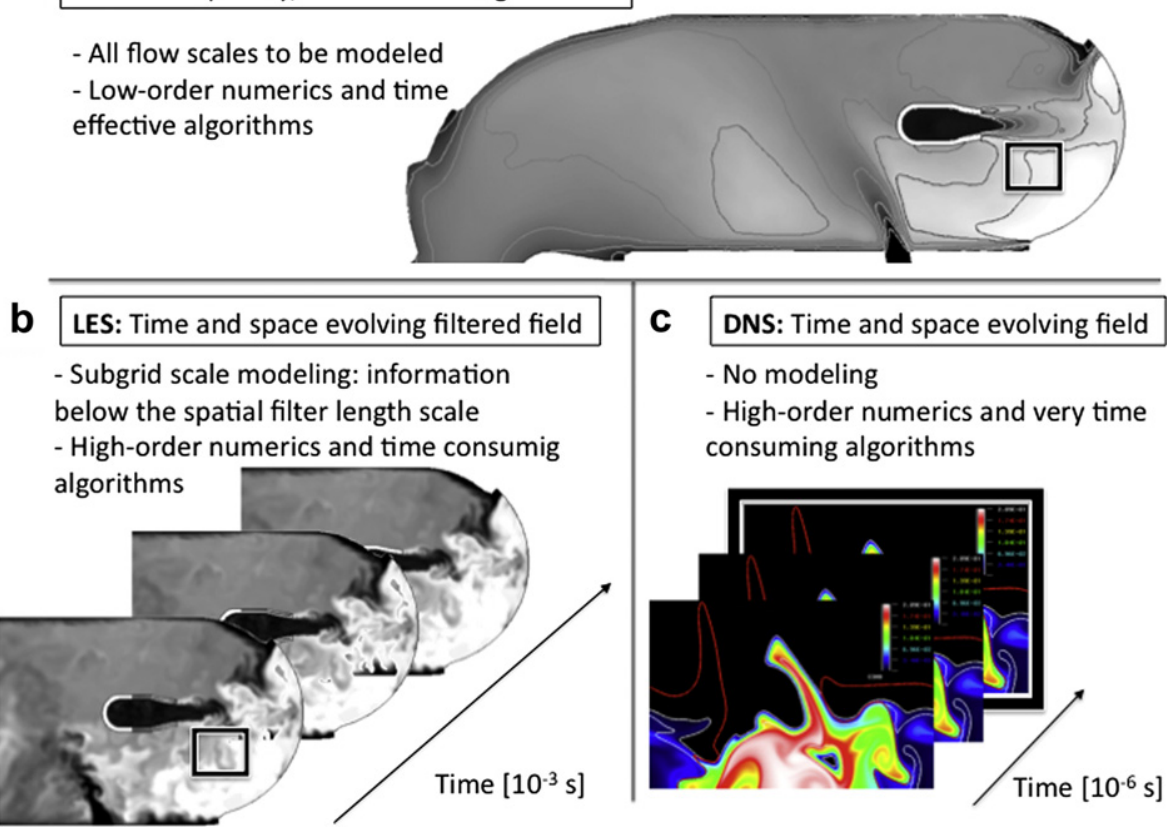

Fig. 1. Schematic representation of the three numerical methods used to simulate turbulent reacting flows: (a) RANS provide access to a temporally/ensemble averaged field representing the flow field in complex systems (extracted from [319]); (b) LES give access to a temporally and spatially evolving set of fields representative of the spatially filtered governing system of equations (extracted from $[320,360]$ ) and (c) DNS provide the exact spatially and temporally evolving field obtained by directly solving the governing equations (extracted from [361]). 
complex predictions. From a modeling point of view, combustion CFD scientists improved numerical predictions by focusing their efforts on time and space dependent descriptions of the problems. The main objective of such unsteady simulation is to relax the modeling constraints by taking into account unsteadiness and inhomogeneities which are very difficult to model with RANS [11-13]. Two fully unsteady computing and modeling strategies are currently available for turbulent reacting flows: (1) Direct Numerical Simulations (DNS) and (2) Large Eddy Simulations (LES). While DNS, Fig. 1(c), suppress any notion of modeling [14-19] aside from the chemical model which needs to be supplied, LES, Fig. 1(b), introduce a scale separation between the large and small scale flow motions [20-30]. Small scale effects on the large scales are thus to be mimicked by a model.

With DNS all scales must be resolved and computing costs grow with the flow turbulent Reynolds and Damköhler numbers [31], respectively noted $R e_{t}=u^{\prime} l_{t} / \nu$ and $D a=\tau_{t} / \tau_{c}$. These two numbers involve the turbulent velocity fluctuation, $u^{\prime}$, its characteristic length scale, $l_{t}$, and time scale, $\tau_{t}$ as well as the dynamic fluid viscosity, $\nu$ and a chemical time scale, $\tau_{c}$. For DNS of non-reacting turbulent flows, every flow scale is to be resolved so scaling laws read: $R e_{t}<N^{(4 / 3)}$, where $N$ is the number of cells in each direction and $R_{t}^{3}<M$, where $M$ is the number of temporal integration steps $[30,32]$. Both criteria are needed to ensure a proper statistical representation of the larger flow scale as well as of the dissipative scales (Kolmogorov length scale, $\eta_{K}[33,34]$ ). For turbulent premixed reacting flows, the spatial scaling is $\operatorname{Re}_{t} D a<(N / Q)^{2}$ for a one-step irreversible reaction, $Q$ being the number of grid points in the thin reaction zone (of the order of 20 for simple chemical schemes) [32]. DNS is hence limited by two ratios: $l_{t} / \delta_{l}^{0}$, the turbulent to flame thickness ratio and $u^{\prime} / s_{1}^{0}$, the turbulent to chemical speeds. For DNS of turbulent non-premixed reacting flows, mixing and chemical times need to be both accurately represented since $\tau_{c}$ is controlled not only by the mixing of the adjacent streams of fuel and oxidizer but also by the consumption rate (that locates around the stoichiometric line). However both phenomena are flow dependent and clear numerical constraints are difficult to obtain unless more constraints are provided [35-37]. Current computing strategies [37-41] with adaptive meshing and high-order numerical schemes [42-46] allow to cover simple configurations which are used for model validations and understanding of fundamentals of turbulent reacting flows $[43,46,47]$. Aeronautical reactive flows remain out of reach because of the high Reynolds number, $O\left(10^{8}\right)$, and the highly energetic fuels yielding $l_{t} / \delta_{l}^{0}$ to be of the order of 10 to 1000 and $u^{\prime} / s_{l}^{0}$ to range from 0.5 to 500 depending on the target application.

LES put less stringent limits for the computational size by filtering out all flow small scales. Ideally for non-reacting flows, Sug-Grid Scale (SGS) velocity models impose that the scale separation or filter cut-off frequency lies in the inertial range of the turbulent spectrum yielding $R e_{t}<(q N)^{(4 / 3)}$ where $q$ is the proportionality factor between $\eta_{K}$, the Kolmogorov length scale, and the cut-off length of the filter, $\Delta_{F}$. Hence, and aside from the chemistry problem, LES allow simulating turbulent flows with turbulent Reynolds numbers approximately 500 times larger than DNS with the same number of points. Evaluation of the proper scaling for turbulent reacting LES remains unclear and often depends on the turbulent combustion model used to close the corresponding SGS terms.

Over the two last decades, DNS and LES have grown very rapidly thanks to the large increase in computing power and the rise of massively parallel architectures. LES codes and models have appeared as a clear scientific alternative to RANS and they are routinely being developed and bench-marked by the turbulent combustion scientific community. Recent developments of this approach now focus on transient flow phenomena with added complexities: i.e. multi-phase flows, ignition and extinction sequences.... Note however that numerical and modeling prerequisite conditions are usually not available in the literature especially for real aeronautical burner simulations. The actual contribution of such methods and their potential use in the context of the industrial design chain are thus still to be investigated and tested.

This review intents to highlight available strategies and models that have allowed LES of aeronautical applications as well as their validations thereby underlying the current status and potential limitations or need for developments [48]. To do so the document focuses only on recent years gaseous reacting LES in light of the industrial need. Details on LES (modeling, numeric, massively parallel computations...) are first provided. Specificities related to industrial flow burners are then given along with the step-by-step recent LES validations for industry-like experimental burners. The advent of highly resolved LES (almost DNS-like computations) are specifically discussed for these simplified yet complex burners and issues pertaining to the modeling hypotheses of LES sub-models are underlined. Real burner LES are then reviewed to yield the industrial state-of-the-art and highlight potential directions for future developments.

\section{Fundamentals of LES for complex burner simulations}

This section describes the basics of LES: i.e. fundamentals, models for very high Reynolds number flows and the limiting case of low Reynolds number flows, discretization of the governing model equations, boundary conditions and their impact on the predictions. A specific subsection is dedicated to the current stateof-the-art computing facilities and their impact on LES strategies.

\subsection{The filtering approach: implicit, explicit and no-model approaches}

LES usually rely on spatial filtering where a filter $\mathcal{G}\left(\mathbf{x}, \mathbf{x}^{\prime} ; \mathbf{t}\right)$, not yet defined, is convoluted with the instantaneous evolution equations or flow variables. Explicit and implicit approaches yield different classes of LES: the former introduces the filter explicitly, applies it to the governing equations and then discretizes the problem to obtain the solution numerically; the latter associates the discretization of the governing equations by an under-resolved grid to a filtering procedure which is undetermined hence implicit. This section describes the differences between the two approaches and highlights their links with the numerical schemes. Details about the closure problem and the current LES models available are given in the following sub-sections.

Filtering can be performed in the space or frequency domains. Although LES in the frequency domain have interesting properties, their use in industry-like configurations is unrealistic. The discussion is limited here to spatially dependent filters and temporally invariant functions [49]: i.e. $\mathcal{G}\left(\mathbf{x}, \mathbf{x}^{\prime} ; \mathbf{t}\right)=G\left(\mathbf{x}-\mathbf{x}^{\prime} ; t\right)$. To ease manipulation of the governing equations, the filter is usually selected to satisfy the conservation of constants (linearity), local invariance and whenever possible to commute with derivation $[30,50]$. These constraints are strong and often do not apply to bounded non-uniform non-homogeneous flow problems. Commutation errors are usually imbedded in real flow LES formalisms [51] unless specific filters are introduced [52-55]. To ensure a proper flow representation, generic properties of the initial set of Navier-Stokes equations for reacting mixtures are also to be conserved. Typically, Galilean, time, rotational, refection invariances and material indifference [56-58] of the filtered quantities are desirable. The unfiltered Navier-Stokes equations satisfy these constraints but the LES equations may not because of the models proposed as closures. For reacting flows, the most 
important property is probably the need to conserve bounds to ensure that filtered species mass fractions do not go above one and below zero. That simple mathematical property translates in the need for positive filters.

Although the notion of filters is mastered in the mathematical context, its use in LES codes is not well defined (see chapter 7 of [30] for details). The previous discussions on the desired filter properties are justified in the explicit filtering context: i.e. the filter is well defined and the governing equations are the result of the convolution operation. However, solving for the filtered transport equations requires numerical schemes that solve a discrete representation of the continuous problem. This discretization introduces new spatial scales into the problem especially if the grid is nonuniform (typical of industrial LES solvers). Changes of cell topologies or mesh stretching are known problems from the purely numerical point of view. In a fully explicit LES approach, the discretization should not introduce un-controlled uncertainties so that high-order non-dissipative and non-dispersive schemes are mandatory [59]. Their use whenever possible however increases the computer cost of such simulations. To reduce spurious numerical oscillations implicit filtering or pre-filtering can be introduced in the simulation (at every time step for example) $[58,59]$ but again an additional overhead is inferred. Theoretically, the notion of effective filter issued by a simulation can be introduced [23] irrespectively of the numerics or filter used to derive the governing equations. In this context, LES can be viewed as a combination of one subgrid model and one numerical scheme, leading to an unknown filter also called 'effective filter' $[30,60]$. The fact that subgrid models and numerical schemes are intrinsically linked has lead to alternative solutions where all the dissipation is provided by the numerics only and no sub-grid model is used $[61,62]$. In that case, "no model" LES or MILES (Monotone Integrated Large-Eddy Simulation) can be performed if the numerical scheme is constructed adequately [63].

Despite various potential frameworks, the risk with all these LES approaches is clear. It essentially stems from the ratio between the simulation inertia forces to the simulation dissipative forces (resolved field convective force over the model plus numerical dissipation forces): if this effective simulation Reynolds number locally depends explicitly on the grid or model and differs from the real flow Reynolds number, large flow differences are expected. Such criteria are particularly critical for transitioning flows or specific regions of a turbulent flows (near wall region). Despite this limit, recent developments prove LES to be a promising tool for complex applications. Its strong ties with numerics and modeling essentially yields a difficult environment for scientists to adequately evaluate and assess potential paths of improvements toward fully controlled and mastered LES at an engineering level.

\subsection{LES transport equations and sub-models}

Due to the non-linear nature of the governing equations of turbulent reacting flows, spatial filtering yields a closure problem where sets of new unknown terms need to be modeled for the problem to be solved numerically [1-10]. Denoting the Favre filtered field by,

$\widetilde{\rho} \tilde{f}(\mathbf{x} ; t)=\int \rho\left(\mathbf{x}-\mathbf{x}^{\prime} ; t\right) f\left(\mathbf{x}-\mathbf{x}^{\prime} ; t\right) G\left(\mathbf{x}-\mathbf{x}^{\prime}\right) \mathrm{d} \mathbf{x}^{\prime}$,

$\rho$ standing for the fluid density, the following recursive properties apply to any quantity $\tau$ combining primitive variables [64],

$\tau(f, g)=\widetilde{f g}-\tilde{f} \tilde{g}$, $\tau(f, g, h)=\widetilde{f g h}-\tilde{f} \tilde{g} \tilde{h}-\tilde{f} \tau(g, h)-\tilde{g} \tau(f, h)-\tilde{h} \tau(f, g)$

These terms and the LES models used for them must satisfy the desired filter properties described in Section 2.1. For turbulent reacting flows, the filtered compressible, multi-species governing LES equations read:

Species $\alpha$ mass fraction, $Y_{\alpha}$, conservation:

$\frac{\partial \bar{\rho} \widetilde{Y_{\alpha}}}{\partial t}+\frac{\partial}{\partial x_{j}}\left(\bar{\rho} \widetilde{Y_{\alpha}} \widetilde{u_{j}}\right)=-\frac{\partial}{\partial x_{j}}\left[\overline{J_{j, \alpha}}+{\overline{J_{j, \alpha}}}^{t}\right]+\overline{\dot{\omega}_{\alpha}}$,

Momentum conservation:

$\frac{\partial \bar{\rho} \widetilde{u}_{i}}{\partial t}+\frac{\partial}{\partial x_{j}}\left(\bar{\rho} \widetilde{u}_{i} \widetilde{u}_{j}\right)=-\frac{\partial}{\partial x_{j}}\left[\bar{P} \delta_{i j}-\bar{\tau}_{i j}-\bar{\tau}_{i j}^{t}\right]$,

Total energy, $E$, conservation:

$\frac{\partial \bar{\rho} \tilde{E}}{\partial t}+\frac{\partial}{\partial x_{j}}\left(\bar{\rho} \tilde{E} \widetilde{u_{j}}\right)=-\frac{\partial}{\partial x_{j}}\left[\overline{u_{i}\left(P \delta_{i j}-\tau_{i j}\right)}+\overline{q_{j}}+{\overline{q_{j}}}^{t}\right]+\overline{\dot{\omega}_{T}}$,

where, $P$ is the pressure, $u_{i}$ the ith component of the flow velocity vector, $J_{j, \alpha}, \tau_{i j}, q_{j}$ are respectively used to denote the species diffusion fluxes, the viscous stress tensor and heat flux [32]. Finally, $\dot{\omega}_{\alpha}$ and $\dot{\omega}_{T}$ are the species source terms and heat release rate issued by the chemical process taking place in the flame. The ideal gas law and mixing laws are also needed to close the problem. In Eqs. (4)-(6), three classes of unknown terms can be distinguished. They involve correlations between velocity components, $u_{i}$, species mass fractions, $Y_{\alpha}$, temperature, $T$, (denoted by the superscript $t$ in Eqs. (4)-(6)) as well as chemical source terms, $\dot{\omega}_{\alpha}, \dot{\omega}_{T}$ :

- Second-order correlations, ${\overline{\tau_{i j}}}^{t}=-\bar{\rho} \tau\left(u_{i}, u_{j}\right),{\overline{J_{j, \alpha}}}^{t}=\bar{\rho} \tau\left(u_{i}, Y_{\alpha}\right)$ $\ldots$ appear when rewriting the convective terms of the filtered governing equations. These quantities are usually associated with the loss of information due to filtering fields containing a large range of length and time scales as encountered in a turbulent flow (i.e. without chemical reaction) $[65,66]$. The associated terms are the so-called Sub-Grid Scale (SGS) Reynolds stresses appearing in the filtered momentum conservation equations, Eq. (5), and the SGS scalar fluxes appearing in the filtered species conservation equations [10,32,67], Eq. (4).

- When solving for the mixture species equations, higher order correlation terms arise from the filtering of the highly non linear chemical reactions that control the consumption and production of species and heat release: i.e. $\overline{\dot{\omega}_{\alpha}}, \overline{\dot{\omega}_{T}}$. These chemical source terms need to be addressed accurately if combustion is to be properly predicted. Such terms involve complex products of species mass fraction to given powers, exponential functions of temperature and they cannot be simply approximated by the substitution in the respective expressions of the filtered fields [68-70]. The art of turbulent combustion modeling is a key ingredient of models for $\overline{\dot{\omega}_{\alpha}}$ and $\overline{\dot{\omega}_{T}}$ : many models actually express source terms as functions of new quantities such as the scalar dissipation rate or the flame surface density, depending on the combustion regime [71-78] and require additional conservation equations.

- Other terms such as $\tau\left(u_{i}, u_{j}, u_{k}\right)$ or $\tau\left(u_{i}, p\right)$ are often disregarded and will not be comprehensively discussed here unless specifically needed. For example, transport of any energy which includes the flow kinetic energy, yields, after filtering, a third order term which needs closure [79]. Compressible flow governing equations also involve pressure-velocity terms that may be of importance [80-82]. Similarly, it is of usual practice to neglect molecular properties fluctuations (such as viscosity, diffusivity...). 
2.2.1. Turbulence models for velocity and scalars

2.2.1.1. SGS Reynolds stress models. Conventional SGS Reynolds stress models are based on the Boussinesq hypothesis and the notion of turbulent viscosity. These are probably the most popular models and almost exclusively used in industrial flow LES. A noncomprehensive view of such closures is provided below with emphasis on their derivation and the target properties, Table 1 . Since real reacting flow LES mainly treat the problem in physical space, only models applicable in this context are addressed, although spectral models do provide important information. For more information or a current status on developments in LES turbulent modeling, readers are referred to more fundamental reviews $[22,25,29,30]$.

The Smagorinsky model [83] is probably the most popular turbulent closure model when dealing with complex configurations because of its simplicity and robustness. Derived in the context of isotropic decaying turbulence [84] with an assumption of equilibrium between kinetic energy fluxes at all turbulent scales, its advantages and weaknesses are well known. Its first weakness is that it is not suitable for transitioning flows or to treat wall turbulence. Improvements of the original model cover its use for anisotropic grids [85,86], automatic estimations of the model constant [87-89] through the use of dynamic procedures based on Germano's identity [64] or the use of a transport equation for the SGS kinetic energy to account for non-equilibrium of the turbulent field [90,91]. Although dynamic procedures clearly improve LES predictions and extend the general use of the SGS closures, their implementation in general purpose LES codes usually requires some local averaging $[88,89,92,93]$ and an implementation of a test filter.

In the context of wall bounded or transitioning flows with a strong impact of the mean shear, the conventional eddy viscosity estimates over-predict the turbulent diffusivity resulting in excessive turbulent diffusion and artificial re-laminarization of the LES filtered field. Based on turbulent properties and invariants [56,94-96], new expressions are possible to improve the model behavior as produced by [30,97-100] similarly to RANS approaches [101]. An alternative for wall bounded flows which still remains a weak point of LES SGS models is the use of correction functions or specific wall modeling [102-105].

A second class of SGS velocity model relies on the similarity hypothesis between the resolved field and a test scale [106].
Linearization of the similarity hypothesis yields the so-called tensorial eddy viscosity model or non-linear model [107]. Deconvolution methods have also appeared [108] and although these new models have shown great potential in a priori validations, a posteriori use proved them to be insufficiently dissipative. Mixed closures relying on the first set of Smagorinsky like models and similarity expressions have been proposed $[109,110]$.

Other approaches [63,86,90,111-117] with various degrees of maturity illustrate the still on-going effort to propose reliable and more general turbulent LES closures.

2.2.1.2. SGS species and scalar flux models. Similarly to the velocity SGS term, the species or scalar SGS flux is usually modeled based on the Boussinesq hypothesis and the use of turbulent Schmidt or Prandtl numbers [118]. Such numbers are necessary to deal with the differences in physics that govern the evolution equations: i.e. turbulent fluctuations cover all scales from integral to Kolmogorov scales [33,119] while scalar fluctuations go all the way to the Batchelor or Corrsin $(S c<1)$ scales $[3,70,120]$. Dynamic procedures have also been proposed [80,121,122] following the formalism discussed above. Extensions using different tensorial relationships have also been developed [123,124].

For species and temperature turbulent mixing, alternatives to the gradient diffusion hypothesis are scarce and mainly reduce to the Linear Eddy Mixing (LEM) model [125-133] or transported FDF approaches $[134,135]$. It is important to note that most of the mixing LES models available today strongly rely on the accuracy of the turbulent viscosity closure. Furthermore these are usually directly applied to turbulent reacting flows despite the clear limitations and the strong link that exists between mixing and chemical reactions $[68,70,136]$.

\subsubsection{Combustion models}

The filtered species and energy equations cannot be closed without a significant modeling effort. They are not only governed by turbulence but also depend on mixing: different combustion regimes are possible depending on the flow configuration, type of fuel and injection system present in the burner [76]. Combustion regimes are usually introduced to characterize the physical processes that dominate a flame and to choose closure models: i.e. flamelet,

Table 1

Tentative classification of the turbulent SGS LES closures available.

\begin{tabular}{|c|c|c|c|c|c|c|}
\hline & \multirow[t]{3}{*}{ Fundamental hypothesis } & \multicolumn{5}{|l|}{ Model properties } \\
\hline & & \multirow[t]{2}{*}{ Near wall behavior } & \multirow[t]{2}{*}{ Solid rotation } & \multirow[t]{2}{*}{ Pure shear } & \multicolumn{2}{|c|}{ Contraction/expansion } \\
\hline & & & & & Axisymmetric & Isotropic \\
\hline \multicolumn{7}{|l|}{ Eddy viscosity closures $^{\mathrm{a}}$} \\
\hline Smagorinsky [83] & Production $=$ dissipation & $O\left(y^{0}\right)$ & 0 & 1 & $\approx 3: 46$ & $\approx 2: 45$ \\
\hline Wale [97] & Production $=$ dissipation & $O\left(y^{3}\right)$ & $\approx 0: 9$ & 0 & $\approx 0: 15$ & 0 \\
\hline Vreman [98] & Production $=$ dissipation & $O\left(y^{1}\right)$ & $\approx 0: 71$ & 0 & $\approx 1: 22$ & 1 \\
\hline Sigma $[100]$ & Production $=$ dissipation & $O\left(y^{3}\right)$ & 0 & 0 & 0 & 0 \\
\hline \multicolumn{7}{|l|}{ Dynamic closures $^{\mathrm{b}}$} \\
\hline Germano $[64,88]$ & Production $=$ dissipation & $O\left(y^{0}\right)$ & \multicolumn{4}{|c|}{ To be determined - strong dependency on the homogenization procedure } \\
\hline \multicolumn{7}{|l|}{ Similarity based closures } \\
\hline Bardina $[106]$ & Scale similarity hypothesis & \multicolumn{5}{|c|}{ To be determined - strong dependency on the homogenization procedure } \\
\hline \multicolumn{7}{|l|}{ Mixed closures } \\
\hline Samgorinsky-Bardina $[109,110]$ & $\begin{array}{l}\text { Scale similarity hypothesis } \\
\text { \& Pro-duction = dissipation }\end{array}$ & \multicolumn{5}{|c|}{ To be determined - strong dependency on the homogenization procedure } \\
\hline \multicolumn{7}{|l|}{ Non-linear closure } \\
\hline $\begin{array}{l}\text { Tensorial viscosity [107], } \\
\text { de- convolution model [108] }\end{array}$ & Production $=$ dissipation & \multicolumn{5}{|l|}{ To be determined } \\
\hline \multicolumn{7}{|l|}{ One or more equation closures } \\
\hline Additional transport eqns [89-91] & Production $\neq$ dissipation & \multicolumn{5}{|l|}{ To be determined } \\
\hline
\end{tabular}


distributed reaction, thickened flame... [76,137]. Laminar flames are the natural starting point to distinguish combustion modes present in real applications: diffusion flames, premixed flames and partially premixed flames. The addition of turbulence is usually introduced through the notion of combustion diagrams to justify the use of a specific turbulent combustion model knowing the combustion mode. Contrarily to RANS, LES models must also degenerate naturally to filtered laminar flames in zones where turbulence is low: they must preserve a large part of the flame structure.

A brief overview of major LES combustion/chemistry submodels is provided below relying on the presentations of [76] and [137]. More comprehensive reviews on this specific problem are available in $[77,78,138-144]$.

2.2.2.1. Chemical description. Combustion is a multi-scale phenomenon involving a broad range of time scales which find their root at the atom level. Starting with the atomic bounds around $10^{-15} \mathrm{~s}$, Arrhenius laws allow to reduce time scales of leading species from $10^{-15} \mathrm{~s}$ to $10^{-10} \mathrm{~s}$ and of the order of seconds for slower reactions. Integrating such sparse systems remains a challenging task especially when they need to be coupled to transport phenomena. To start addressing such issues prior to their use in a CFD code, different methods are available and one needs to choose from a wide range of strategies [137]:

- Constitutive relations: such as Arrhenius laws for rate constants aim at representing atomistic process by a continuum.

- Chemical mechanism reduction: intent to identify most important species and reaction steps in order to decrease significantly the number of species and reactions needed to represent the initial skeletal mechanism. Different techniques exist for reductions: i.e. Quasi-steady state (QSS), Partial Equilibrium (PE), Computational Singular Perturbation (CSP) [145], Intrinsic Low-dimensional Manifold (ILDM) [146,147].

- Stiff chemistry integrators: aim at removing stiffness in the set of ordinary or partial differential equations $[148,149]$ needed to describe the reduced chemical system in the presence of transport.

- Storage chemistry approaches: aim at accelerating the chemistry integration while the CFD integration proceeds. Tabulation of pre-computed laminar or turbulent flames falls in this class of approaches. Flamelet Generated Manifolds (FGM) [150,151] or Flamelet Prolongation of ILDM (FPI) [152-156] tables are typical examples. In Situ Adaptive Tabulation (ISAT) [157], Piece-wise Reusable Implementation of Solution Mapping (PRISM) [158] or Artificial Neural Networks (ANN) [159] are other examples where the tables adapt automatically during the CFD computation.

The main limitation behind all reduction methods is the reduced range of applicability. The final kinetic model also ends up having strong links with the turbulent combustion model which itself contains underlying assumptions (often related to specific combustion modes and regimes). All these observations seriously reduce the extent and generality of some of the tables or schemes obtained.

2.2.2.2. Turbulent combustion models. The turbulent combustion closures available to the CFD LES community are numerous and are often direct extensions of RANS turbulent combustion models. Such direct extensions are legitimate since all scales associated to the flame are usually below the LES filter length scale. Care is however needed as discussed in Section 2.2.3.

Like RANS modeling, LES turbulent combustion models rely heavily on the combustion fundamental theories and combustion modes: i.e. fully premixed, non-premixed [70] for which the basic properties are recalled below.
- Premixed flames are combustion modes where fuel and oxidizer are fully mixed before reacting: the flame front separates the unburnt premixed gases from the fully burnt reactants. Species and temperature transport is important only in the vicinity of the flame. Under the assumption of a simplified chemical description and transport, the governing equations can be recast into a single global equation for the progress variable characterizing the state of reaction. This progress variable is usually noted $c$ and non-dimensionalized to equal zero in fresh gases and one in burnt gases. The unfiltered or exact evolution equation of $c$ reads:

$\frac{\partial \rho c}{\partial t}+\frac{\partial}{\partial x_{j}}\left[\rho u_{j} c\right]=\frac{\partial}{\partial x_{l}}\left[\rho D \frac{\partial c}{\partial x_{l}}\right]+\dot{\omega}_{c}$.

If the flame is thin and can be described as a propagating front, Eq. (7) can be written in a propagative form called the G-equation by tracking the position of an iso-c surface [75]:

$\frac{\partial \rho c}{\partial t}+\frac{\partial}{\partial x_{j}}\left[\rho u_{j} c\right]=\rho s_{L}^{0} \frac{\partial c}{\partial x_{l}}$

where the laminar flame speed noted $s_{L}^{0}$ appears explicitly and characterizes the propagation of the local premixed flame elements [160-162].

- For non-premixed or diffusion flames, fuel and oxidizer are separated and combustion occurs in the diffusive region where molecular and turbulent transport allow mixing of the two components prior to reaction. Here again, laminar and SGS mixing terms are essential since they control the rate of consumption $[70,75,76]$. For simplified transport and kinetics, Schwab-Zeldovich [163] variables or conserved scalars (noted $Z$ ) transport equations can be derived to represent the state of mixing within the flame independently of reaction. Its unfiltered or exact transport equation reads:

$\frac{\partial \rho Z}{\partial t}+\frac{\partial}{\partial x_{j}}\left[\rho u_{j} Z\right]=\frac{\partial}{\partial x_{l}}\left[\rho D \frac{\partial Z}{\partial x_{l}}\right]$.

Similarly to premixed modes, a coordinate attached to the stoichiometric iso- $Z$ surface allows to recast the species transport equations with specific diffusive terms: scalar dissipation rate $\chi=D\left(\partial Z / \partial x_{l}\right)\left(\partial Z / \partial x_{l}\right)$, species transport across iso-Z lines in the normal and tangential iso- $Z$ directions and reaction. Neglecting curvature effects, local composition within the flame appears to be controlled by the scalar dissipation rate.

- All regimes which are nor premixed neither non-premixed are called partially premixed. They are encountered quite often in gas turbines and are much less understood. Such regimes control auto-ignition problems, flame stabilization in the near field of burners, local quenching or re-ignition mechanisms. A simplified partially premixed flame prototype is the triple flame configuration [164-171]. Classical models developed for premixed or purely diffusion flames should not be used unless specifically adapted.

Based on these fundamental developments for specific combustion modes, many turbulent combustion models are available for LES. Following the classification of $[31,76,172]$, one distinguishes between the purely geometrical and pseudo-statistical/ statistical type of closures.

In purely geometrical approaches, the governing equations are in a propagation form and unclosed terms are provided in light of the combustion regimes characterized by the turbulent Reynolds 
number, $R e_{t}$, turbulent Damköhler number, $D a_{t}$ and/or the turbulent Karlovitz number, $\mathrm{Ka}_{\mathrm{t}}$. These models are usually linked to the flamelet assumption: i.e. the reaction zone is thin compared to turbulent length scales and only curvature, stretch and wrinkling effects impact the filtered rate of reaction in the iso- $c$ or iso- $Z$ formulations. For this class of models the notion of flame surface density per unit volume, $\Sigma$, or flame wrinkling factors, $\Xi$, can also be introduced to evaluate the filtered value of the reaction rate [173-178] using: $\widetilde{\dot{\omega}_{k}}=\widetilde{\dot{Q}_{k}} \tilde{\Sigma}$, where $\widetilde{\dot{\Omega}_{k}}$ stands for the local density filtered burning rate of species $k$ per unit flame area, or using a model for $\tilde{\Xi}={\widetilde{\dot{Q}_{k}}}_{k} \sqrt{\left(\partial \tilde{c} / \partial x_{l}\right)\left(\partial \tilde{c} / \partial x_{l}\right)}$, the wrinkling factor. Both approaches require information on the flame inner structure and chemistry.

In statistical models, SGS terms can be constructed using the Filtered Density Function (FDF) or Probability Density Function (PDF) associated with the filtering operation of LES [179] (cf. [180] for further discussions on the differences between FDF and PDF formalisms in LES). The main result of such formalisms is that unknowns can be obtained by direct integration of the FDF or PDF. In these methods, two distinct sub-classes are to be distinguished:

- The presumed approach where the FDF/PDF form is fixed a priori and usually parameterized by the local value of the filtered quantity and its second filtered central moments. Conventional presumed PDF shapes are the delta, beta, Gaussian and Log-Normal functions. Within the same working frame, the Conditional Filtered Moment Closure (CMC) [137,181] approach consists in solving the transport equations of the conditionally filtered terms (some of which appear in the evolution equation of the FDF/PDF) which can then be multiplied by a presumed FDF/PDF and integrated to yield estimates of the SGS unknowns.

- The transported FDF/PDF approach solves directly for the governing equation of the function and performs the numerical integration of the estimated FDF/PDF to yield values for the filtered unclosed terms present in the LES transport equations.

From a modeling point of view each approach implies the closure of specific terms appearing in the evolution equations. In that respect, SGS or equivalent terms involving mixing through velocity/species are to be addressed unless the velocity/species FDF/PDF is considered [113,134,135,182-185]. Scalar dissipation rate or equivalent terms must be closed too $[67,74,186]$.

One last type of model for LES of turbulent reacting flows can also be somehow classified as pseudo-statistical approach: the Linear Eddy Model (LEM) [131,132]. In this approach, the initial filtered profiles (or coarse-grained structures) are mapped onto a so-called triple-map structure which is parameterized by a PDF and aims at representing the effect of eddies on mixing as well as stirring all the way to the viscous range. Reaction is taken into account in this 1-D space. Each physical process is taken into account using a splitting operator technique preserving specific time scales of each process.

Table 2 summarizes possible LES turbulent combustion models with specificities and context of development. For further information, readers are redirected to $[76,137]$ and turbulent combustion books $[31,75,119,187]$.

\subsubsection{LES and the DNS limit modeling constraint}

Originally LES models were constructed for non reacting flows and derived in the high Reynolds number limit to ensure the existence of the inertial range within the turbulent spectrum. Another constraint is that LES model contributions should vanish as the filter size tends to zero to recover the DNS limit of the simulations: i.e. a fully resolved simulation where all of the dissipative scales are captured. Likewise and to properly recover the DNS limit or transition regions, models should distinguish regions of potential low Reynolds number from fully turbulent ones, go to zero near walls and flows in solid rotation or purely sheared, in axisymmetric or isotropic expansion (or contraction). With such constraints dynamic filtering $[64,85,87,88,188]$ and

Table 2

Tentative classification of the turbulent reacting LES closures available.

\begin{tabular}{|c|c|c|c|c|c|}
\hline Formalism & Modeling & Solved quantity & Closures & Reaction source term & Range of application \\
\hline \multirow[t]{4}{*}{ Geometric } & G-field & $G$ & $\overline{S_{T}}$ & $\overline{\partial \tilde{G} \partial \tilde{G}}$ & Premixed \\
\hline & Flame surface density & $\widetilde{\sum}$ & $\widetilde{\dot{\Omega}_{k}}$ & $\widetilde{\tilde{\theta}} \widetilde{\dot{\theta}}$ & All modes \\
\hline & Flame wrinkling & $\tilde{c}$ & $\tilde{\Xi}$ & 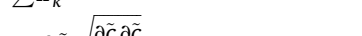 & All modes \\
\hline & Thickened flame & $\tilde{Y}_{k}$ & $E$ (Efficiency function) & $\frac{E \dot{\omega}_{k}\left(\tilde{Y}_{k}, \tilde{T}\right)}{\mathcal{F}}(\mathcal{F}$, thickening factor $)$ & All modes \\
\hline \multirow[t]{10}{*}{ Statistical } & Presumed FDF/PDF & $\tilde{Z} ;(\widetilde{Z Z}-\tilde{Z} \tilde{Z})$ & $\left(\widetilde{u_{i} Z}-\tilde{u}_{i} \tilde{Z}\right) ; D \frac{\partial \bar{Z}}{\partial x_{k} \partial x_{k}}-D \frac{\partial \tilde{Z}}{\partial x_{k}} \frac{\partial \tilde{Z}}{\partial x_{k}}$ & $\int \dot{\omega}_{k}(Z) f(Z) \mathrm{d} Z$ & Non-premixed \\
\hline & & $\tilde{c} ;(\tilde{c} c-\tilde{c} \tilde{c})$ & $\left(\widetilde{u_{i} c}-\tilde{u}_{i} \tilde{c}\right) ; D \frac{\partial c}{\partial x_{k} \partial x_{k}}-D \frac{\partial \tilde{c}}{\partial x_{k}} \frac{\partial \tilde{c}}{\partial x_{k}}$ & $\int \dot{\omega}_{k}(c) f(c) \mathrm{d} c$ & Premixed flames \\
\hline & & All of the above & All of the above & $\iint \dot{\omega}_{k}(c, Z) f(c, Z) \mathrm{d} c \mathrm{~d} Z$ & All modes \\
\hline & CMC & $\widetilde{Y_{k} \mid c}$ & $\widetilde{u_{i} \mid c} ; \widetilde{u_{i} Y_{k} \mid c} ; D \frac{\partial Y_{m}}{\partial x_{l}} \frac{\partial Y_{n}}{d x_{l}} \mid c$ & $\int \overline{\dot{\omega}_{k} \mid c} f(c) \mathrm{d} c$ & Premixed \\
\hline & & $\widehat{Y_{k} \mid Z}$ & $\widetilde{u_{i} \mid Z} ; \widetilde{u_{i} Y_{k} \mid Z} ; D \frac{\partial Y_{m}}{\partial x_{l}} \frac{\partial Y_{n}}{d x_{l}} \mid Z$ & $\int \overline{\dot{\omega}_{k} \mid Z f}(Z) \mathrm{d} Z$ & Non-premixed \\
\hline & & $Y_{k} \mid Z, c$ & $\widetilde{u_{i}}\left|Z, c ; \widetilde{u_{i} Y_{k} \mid Z}, c ; D \frac{\partial \widetilde{Y_{m}}}{\partial x_{l}} \frac{\partial Y_{n}}{d x_{l}}\right| Z, c$ & $\iint \overline{\dot{\omega}_{k} \mid c, Z} f(c, Z) \mathrm{d} c \mathrm{~d} Z$ & All modes \\
\hline & LEM & $\tilde{Y}_{k}$ & $f(l)$ & $\overline{\dot{\omega}_{k}\left(Y_{k}\right)}$ & All modes \\
\hline & Transported FDF/PDF & $f(\xi)$ & $\widetilde{u_{i} \mid \xi} ; D \frac{\partial \mathcal{C}}{\partial x_{l}} \frac{\partial c}{\partial x_{l}} \mid \xi$ & $\int \hat{\omega}_{c}(\xi) f(\xi) \mathrm{d} \xi$ & Premixed \\
\hline & & $f(\eta)$ & 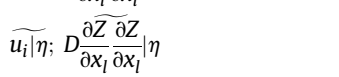 & $\int \dot{\omega}_{k}(\eta) f(\eta) \mathrm{d} \eta$ & Non-premixed \\
\hline & & $f\left(\psi_{k}\right)$ & $\widetilde{u_{i} \mid \psi_{k}} ; D \frac{\partial Y_{m}}{\partial x_{l}} \frac{\partial Y_{n}}{\partial x_{l}} \mid \psi_{k}$ & $\int \dot{\omega}_{k}\left(\psi_{j}\right) f\left(\psi_{j}\right) \mathrm{d} \psi_{j}$ & All modes \\
\hline
\end{tabular}


higher order tensor relationships constitute clear benefits since introducing increased locality through test filtering or improved asymptotic behaviors of the SGS modeling issued by a better qualification of the local resolved flow state, Table 1.

For reacting flows, the situation is more complicated because combustion almost always takes place at scales which are not resolved today. For example, LES of experiments with low Reynolds but large Damköhler numbers corresponds to flows where all turbulent scales can be resolved but the flame front reaction zone is still under-resolved. Typically, in real applications the fuel consumption rate of highly energetic species such as kerosene is large and leads to Damköhler numbers larger than one (i.e. very thin reaction fronts). Within the same flow, the Damköhler number associated with the chemical steps of $N O$ or $C O$ are usually close to one. Modeling is thus confronted with the major difficulty of ensuring the proper description of the interaction of the kerosene consumption in a thin front strongly affected by turbulence while providing the missing information for slowly evolving chemical reactions in an inhomogeneous hot medium that is weakly turbulent. Modeling is thus essential: even-though models are usually derived for flames in highly turbulent flows, they must also be able to propagate quasi-laminar fronts in low turbulence zones as well as in highly resolved intense turbulent fields. In some configurations, neglected terms in the exact filtered transport equations may play non-negligible roles [189-192]. Such issues are becoming of greater importance with the advent of massively parallel computers: in a few years, these computing resources will allow to resolve flow and flame structures in such regimes and SGS models must degenerate to true DNS [189-192].

A final observation which further underlines the potential difficulty of mastering the DNS limit of LES comes from recent works on LES modeling for stationary turbulent flows [193-196]. From these studies, it is clear that defining a clear procedure to unanimously qualify different LES approaches is not an easy task. Indeed, it is now well accepted that a LES prediction is the result of combined modeling and numerical errors and the behavior of such a cocktail is often counter-intuitive [193-196]. For example, if one accepts to change the value of the Smagorinsky constant noted $C_{S}$, an optimal grid resolution $C_{S}$ pair exists which provides good quality predictions $[193,194,196]$ at minimum cost and away from the DNS limit. Because of such observations, multiple LES quality indices have been proposed [193,197-199] for non-reacting and reacting [200] flows. These issues are still to be answered to systematically qualify LES SGS modeling and numerical strategies for an improved understanding of LES predictions in complex geometries.

\subsection{Numerical methods}

LES flow solvers are either incompressible, fully compressible or based on the low Mach number approximation. Each approach imposes different algorithms, computer costs and numerical schemes which may not be compatible with LES basic constraints. However and based on current state-of-the-art LES solvers, key features seem to emerge and this section summarizes them with a specific emphasis on their advantages and disadvantages. Readers can find details associated to High Performance Computing for CFD on massively parallel systems in recent review papers [201,202]. A non-comprehensive list of codes dedicated to LES of reacting flows is provided in Table 3 along with the numerical characteristics retained in each case and the research groups involved in their developments.

\subsubsection{High-order schemes, mesh type and complex geometries}

Despite recent controversies, there is little doubt that high-order schemes are desirable for LES to minimize numerical dispersion and

Table 3

Tentative survey of the massively parallel codes available for complex reacting LES applications.

\begin{tabular}{|c|c|c|c|}
\hline Code & Formalism & Numerics & Target burners \\
\hline PUFFIN (Loughborough University) & $\begin{array}{l}\text { Low Mach, conserved scalar/progress } \\
\text { variable Navier-Stokes }\end{array}$ & $\begin{array}{l}\text { Second order in time and space, } \\
\text { multi-block structured code }\end{array}$ & Lab-scale burners \\
\hline FLOWSI (Imperial College) & $\begin{array}{l}\text { Low Mach, conserved scalar/progress } \\
\text { variable Navier-Stokes }\end{array}$ & $\begin{array}{l}\text { Second order in time and space, } \\
\text { multi-block structured code }\end{array}$ & Lab-scale burners \\
\hline BOFFIN (Imperial College) & $\begin{array}{l}\text { Low Mach, conserved scalar/progress } \\
\text { variable Navier-Stokes }\end{array}$ & $\begin{array}{l}\text { Second order in time and space, } \\
\text { multi-block body fitted structured code }\end{array}$ & Lab- and real-scale burners \\
\hline $\begin{array}{l}\text { FASTEST 3D (Technical University } \\
\text { of Darmstadt) }\end{array}$ & $\begin{array}{l}\text { Low Mach, conserved scalar/progress } \\
\text { variable Navier-Stokes }\end{array}$ & $\begin{array}{l}\text { Second order in time and space, } \\
\text { multi-block body fitted structured code }\end{array}$ & Lab-scale burners \\
\hline CDP (Stanford University) & $\begin{array}{l}\text { Low Mach, conserved scalar/progress } \\
\text { variable Navier-Stokes }\end{array}$ & $\begin{array}{l}\text { Second order in time and space, } \\
\text { unstructured meshes }\end{array}$ & Lab- and real-scale burners \\
\hline $\begin{array}{l}\text { NGA (Stanford University; University } \\
\text { of Colorado; Cornell University) }\end{array}$ & $\begin{array}{l}\text { Low Mach, multi-species (transported } \\
\text { PDF) Navier-Stokes }\end{array}$ & $\begin{array}{l}\text { Second order in time and space, } \\
\text { multi-block structured code }\end{array}$ & Lab-scale burners \\
\hline RAPTOR (Sandia National Lab. code) & Compressible, multi-species Navier-Stokes & $\begin{array}{l}\text { Second order in time and space, } \\
\text { multi-block structured code }\end{array}$ & Lab- and real-scale burners \\
\hline LESLIE (Giorgia Inst. of Tech.) & $\begin{array}{l}\text { Compressible multi-species, conserved } \\
\text { scalar/progress variable Navier-Stokes }\end{array}$ & $\begin{array}{l}\text { Second order in time, fourth order } \\
\text { in space, multi-block hybrid (structured, } \\
\text { unstructured) code }\end{array}$ & Lab- and real-scale burners \\
\hline Penn. State University code & Compressible, multi-species Navier-Stokes & $\begin{array}{l}\text { Second order in time and space, } \\
\text { multi-black structured code }\end{array}$ & Lab-scale burners \\
\hline YALES2 (CORIA) & $\begin{array}{l}\text { Low Mach, conserved scalar/progress } \\
\text { variable Navier-Stokes }\end{array}$ & $\begin{array}{l}\text { Explicit, third order in time and space, } \\
\text { unstructured/hybrid meshes }\end{array}$ & Lab- and real-scale burners \\
\hline AVBP (CERFACS) & Compressible, multi-species Navier-Stokes & $\begin{array}{l}\text { Explicit, third order in time and space, } \\
\text { unstructured/hybrid meshes }\end{array}$ & Lab- and real-scale burners \\
\hline PRECISE (Rolls-Royce) & $\begin{array}{l}\text { Low Mach/compressible, conserved } \\
\text { scalar/progress variable \& multi-species } \\
\text { Navier-Stokes }\end{array}$ & $\begin{array}{l}\text { Third order in time and space, } \\
\text { unstructured meshes }\end{array}$ & Lab- and real-scale burners \\
\hline $\begin{array}{l}\text { THETA (Deutsches Zentrum fuer } \\
\text { Luft- und Raumfahrt e. V -DLR) }\end{array}$ & Multi-species Navier-Stokes & $\begin{array}{l}\text { Second order in time and space, } \\
\text { unstructured meshes }\end{array}$ & Lab- and real-scale burners \\
\hline OpenFoam & $\begin{array}{l}\text { Low Mach/compressible, conserved } \\
\text { scalar/progress variable \& multi-species } \\
\text { Navier-Stokes }\end{array}$ & $\begin{array}{l}\text { Second order in time and space, } \\
\text { unstructured meshes }\end{array}$ & Lab- and real-scale burners \\
\hline FLUENT (ANSYS) & $\begin{array}{l}\text { Low Mach/compressible, conserved } \\
\text { scalar/progress variable \& multi-species } \\
\text { Navier-Stokes }\end{array}$ & $\begin{array}{l}\text { Second order in time and space, } \\
\text { unstructured meshes }\end{array}$ & Lab- and real-scale burners \\
\hline
\end{tabular}


dissipation to preserve good quality unsteady flow predictions. That constraint imposes the use of high-order centered spatial schemes with the addition of artificial viscosity on highly disturbed grids. In a world where only simple geometries would be computed (for example laboratory-scale systems), this constraint would lead to structured grid methods on which high-order schemes (from 4 to 8th order) are easily built. Unfortunately, most real combustion chambers have geometries which are so complicated that meshing them with a multi-block structured grid takes too much time. In addition, good strong scaling requires balanced decomposition of the computational domain which is difficult with a predecomposed block-structured approach in geometries other than cubes and assimilated. As a result, most recent LES codes for real gas turbines are developed using unstructured or hybrid grids. Interestingly, on such grids, developing high-order numerical schemes is a challenge. Most existing solvers on unstructured grids are limited to second-order spatial accuracy except the (expensive) TTGC scheme developed by Oxford and CERFACS [203]. Combining the accuracy of high-order schemes developed for structured grids with the flexibility of unstructured grid solvers is a key issue in the construction of combustion LES solvers.

\subsubsection{Implicit versus explicit time integration, incompressible, low} Mach and fully compressible approaches

Combustion codes are easily written in compressible form: a simple explicit technique allows to develop rapidly a solver for LES of reacting flows on unstructured grids. The main disadvantage of such solvers is that their time step is limited by the CFL condition which is controlled by the sound speed. For very slow flames, computing a flow-through time can require too many time iterations and lead to a very slow computation (or large turn around time). This discussion is actually complicated by different issues:

- Alternative solutions to explicit compressible codes are: (1) time implicit compressible methods and (2) low-Mach formulations. In time implicit compressible methods, the full compressible equations are solved implicitly to remove the CFL constraint. In low-Mach formulations, acoustics are removed from the conservation equations and a Poisson solver is needed to obtain pressure at each instant. For approaches (1) and (2), large implicit systems must be solved at each iteration. Both approaches are found in combustion codes [204,205] and constitute interesting and efficient alternative methods.
- The fully compressible solvers still have a few advantages $[201,206]$ : being explicit, they are efficient on very massively parallel systems. They also capture acoustics naturally, a property which is mandatory to study certain instabilities. In practice, implicit compressible solvers are stable over a wide range of CFL numbers but they are not precise and must be run for constrained CFL values (smaller than 10), making them potentially as slow as explicit solvers. The main reason for such a behavior is linked to the matrix inversion which is time consuming. Low-Mach number codes offer more convincing performances especially for very slow flames where acoustics is not important and the linear system to be solved of reasonable size.

- In practice, in most gas turbine combustion chambers, Mach numbers are not small. Within swirlers, Mach numbers of the order of 0.3 are common. At chamber outlets, a throat created by the high pressure stator usually creates a choked region. For these cases, compressible solvers remain mandatory.

\subsubsection{Boundary condition treatment and stability}

The numerical treatment of the flow boundary conditions is also of importance and can result in artificially stable or unstable computations. These treatments differ depending on the set of equations solved (i.e. compressible or incompressible Navier-Stokes equations). An important conclusion reached in the last ten years is that the flow within the combustion chamber can be computed with much more precision using LES than RANS and that, at this level of precision, outlet and inlet conditions become critical. Typically, a proper LES of a combustion chamber should include a description of the mean and turbulent flow at the inlet (resolved in time). Moreover, the acoustic impedances at inlet (compressor side) and outlet (turbine side) should also be known: this is not the case today and work is required on these questions because these boundary conditions probably control the flow more than the details of the SGS models used within the chamber.

\subsection{The massively parallel context, mesh generation and data management}

Real burner LES imply the use of high end massively parallel super-computers which process data subsets of the same problem in parallel. Efficient and minimum exchanges of information are mandatory to ensure scalability up to thousands of cores. Message

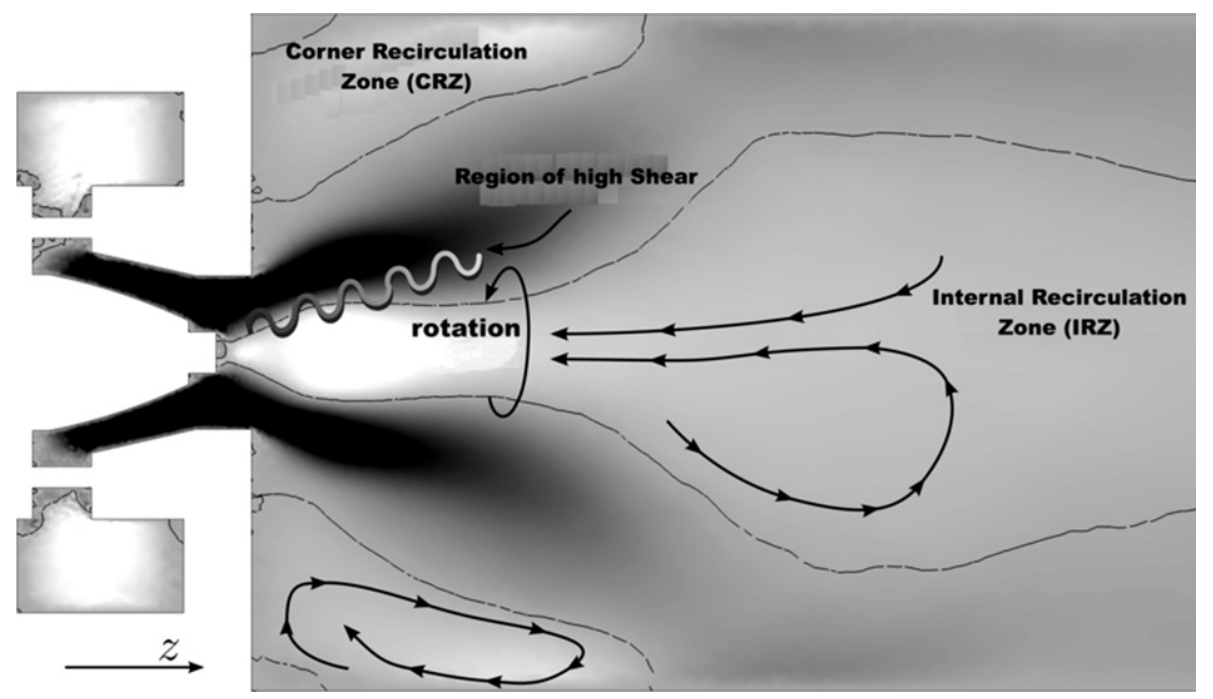

Fig. 2. Typical view of the main flow structures present outside a swirler from a real aeronautical burner provided by Turbomeca and mounted on an experimental test bench [362-364]. 


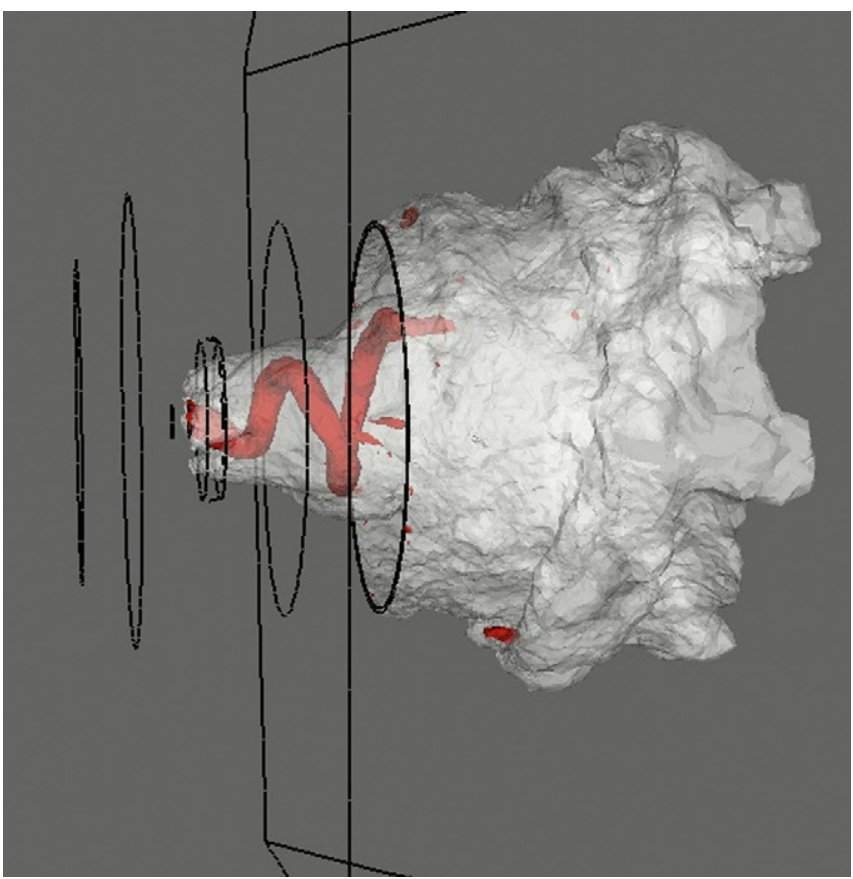

Fig. 3. Typical view of a PVC (red iso-surface) as provided by the LES of a real gas turbine combustion chamber. The light grey iso-surface depicts the flame position. (For interpretation of the references to color in this figure legend, the reader is referred to the web version of this article.) passing coding is at the root of parallel computing. As mentioned above, efficient message passing for CFD in gas turbine chambers requires using fully unstructured grids. However, the generation of large unstructured meshes and their partitioning become bottlenecks today [201,202,207]. I/O must also be totally reorganized to avoid slowing down the code. These issues raise specific questions about the exploitation and maintenance of the codes: maintaining a code which can run efficiently both on distributed and shared memory machines with thousand of cores is extremely difficult.

All of the above mentioned modeling and algorithmic issues are clearly of major importance for real applications. Building codes aiming at simulating real industrial problems often imposes a list of sacrifices: spatial accuracy and modeling are usually sacrificed to ensure robustness and performance, thereby giving access to LES predictions of such complex flows with a bounded and reasonable computer cost. These choices are clearly needed and based on specific and scientifically identified pros and cons; simple models may be favored provided that their limits are well understood and implications on the LES predictions well anticipated. The difficulty inherent to such choices and model limitations are thus of foremost importance to ensure valuable exploitation and understanding of the obtained results to contribute to the decision making. The next section reviews aspects of the validation steps followed by researchers and industry to better qualify the different LES numerical and modeling strategies available today.

\section{Laboratory-scale burner simulations}

Laboratory-scale burners are usually designed to assess modeling and fundamental flow aspects in controlled

Table 4

Tentative survey of laboratory scale burner LES's.

\begin{tabular}{|c|c|c|c|c|}
\hline Ref. & Code & Turbulence & Combustion & Target applications \\
\hline [231] & FLOWSI & Dyn. Smag. & Non-reacting & Bluff body flame \\
\hline$[229,230,232,233]$ & Unknown & Dyn. Smag. & Non-reacting & Swirl flames series \\
\hline [241] & Unknown & Smag. & Non-reacting & TECFLAM burner \\
\hline$[242-244,250]$ & Penn. State & Smag. & Non-reacting & Swirl Mixer (US Patent) \\
\hline [370] & CDP & Dyn. Smag. & $Z, c$, flamelet & Non-prem. coaxial jet comb. \\
\hline [181] & Unknown & & CMC & Bluff body \\
\hline$[371,184]$ & Unknown & Smag. & FDF & Bluff body \\
\hline$[372-375]$ & Unknown & Dyn. Smag. & $c$, flamelet & Swirl flames series \\
\hline$[376]$ & LESLIE & LDKM & LEM & Swirl flames series \\
\hline [377] & PRECISE & Smag. & FDF & Swirl flames series \\
\hline [378] & SiTCom & Smag. & PCM-FPI & Lifted flame, vitiated coflow \\
\hline [379] & LESLIE & LDKM, Mixed & Flamelet $G$, EDC finite rate & Swirl stratified prem. flame \\
\hline [302] & AVBP & Wale & Thick. Flame, reduced chem. & Prem. swirl comb. \\
\hline$[275,276]$ & AVBP & Wale & PCM-FPI & Prem. swirl comb. \\
\hline$[192,205]$ & YALES2 & Dyn. Smag. & C, tabulated chem. & Prem. swirl comb. \\
\hline [190] & AVBP & Wale & FTACLES - FPI & Prem. swirl comb. \\
\hline [247] & AVBP & Smag. & Thick. Flame & Prem. swirl comb. \\
\hline [380-382] & Unknown & LDKM & Reduced chem., wrinkling [383] & GE LM 6000 comb. \\
\hline [384] & Unknown & Smag. & $c$, flame wrinkling, flamelet & Prem. swirl comb. \\
\hline [385] & Unknown & Smag. & $G$ and $Z$, flamelet & Partially prem. comb. \\
\hline [381] & Unknown & Unknown & G & Swirl comb. \\
\hline$[246,386]$ & AVBP & Smag. & Thick. Flame, reduced chem. & Siemens burner \\
\hline$[387,293]$ & AVBP & Smag. & Thick. Flame, reduced chem. & Prem. staged burner \\
\hline [388-391] & Penn. State & Smag. & $G$, flamelet & Prem. swirl comb. \\
\hline [392] & LESLIE & LDKM & Reduced chem. & GE DACRS comb. \\
\hline$[393,394]$ & Unknown & Smag. & EBU, reduced chem. & DOE-NETL prem. comb. \\
\hline$[395,396]$ & LESLIE & LDKM & $G$ and $Z$, flamelet & Prem. comb. \\
\hline [397] & Unknown & Smag. & Level-set, flamelet & Prem. comb. \\
\hline [384] & Unknown & Smag. & Flamelet & Swirl comb. \\
\hline [337] & Unknown & LDKM, Mixed & $Z, c$, flamelet [383] & TARS burner [264] \\
\hline$[398,399]$ & Unknown & Smag. & $G$, flamelet & GE-LM 6000, DOE-HAT \\
\hline$[400,401]$ & LESLIE & LDKM & LEM-LES, reduced chem. & GE-1 \\
\hline$[402]$ & AVBP & Smag. & Thick. Flame, reduced chem. & Alstom swirl \\
\hline$[296,303,304]$ & AVBP & Smag. & Thick. Flame, reduced chem. & Siemens stratified \\
\hline
\end{tabular}


configurations. They are necessary to assess LES strategies as well as their limitations, reliability, capability and orient future developments.

\subsection{Key geometrical features}

To be representative of real gas turbines, laboratory burners need to retain specific features and cover a wide range of turbulent flows and physics that are present in gas turbine engines. For example, it is desirable to use real swirlers and to mount them on experimental test facilities heavily equipped with flow and combustion diagnostics. Since 2000, multiple laboratories have followed this path. In the following mainly swirled flames are discussed as they are typical of the next generation of gas turbine combustion chambers. Swirl is used to generate large recirculation zones that are usually located right outside the injection system, improve mixing, ease flame stabilization and locally increase the flow residence time thereby reducing the size of the combustion chamber $[208,209]$. Simple laboratory burners [208,210-221] with such features have been used to qualify LES.

\subsection{Flow validations}

Swirling flows without combustion have been heavily investigated (see reviews $[208,222-228]$ ). They nonetheless remain

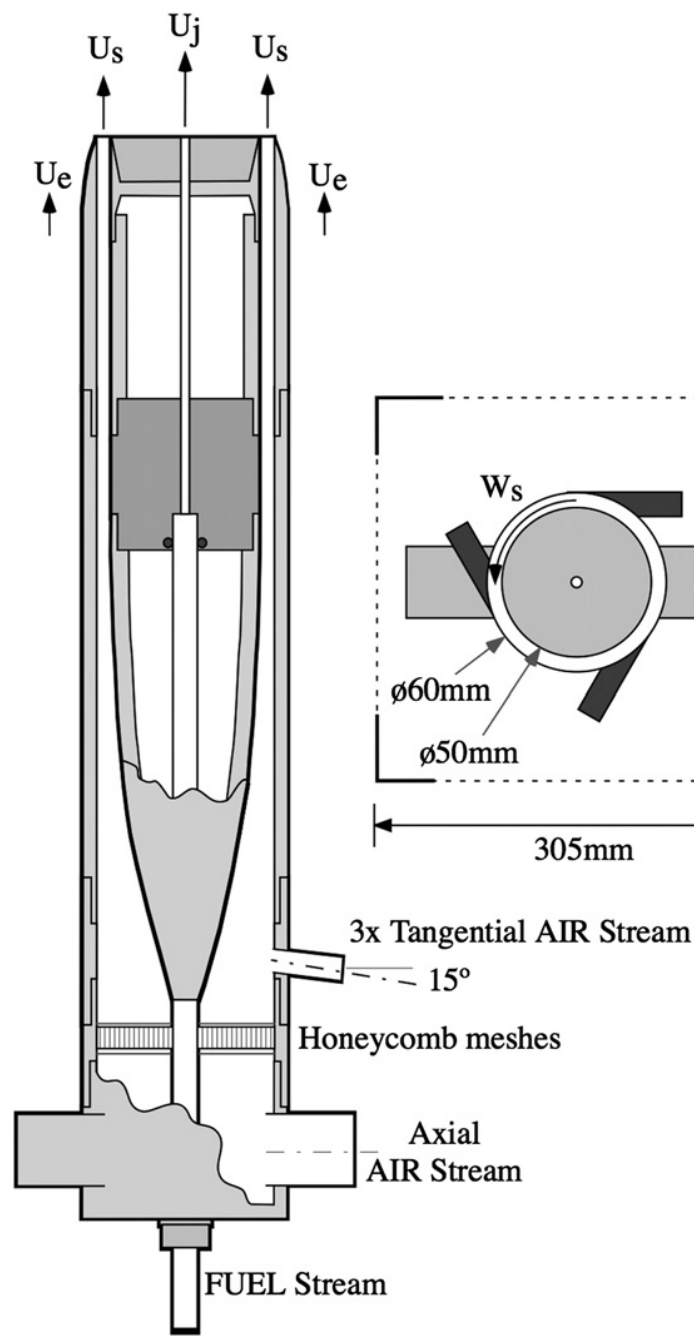

Fig. 4. Geometrical setup of the Sandia swirl combustor used to validate LES $[215,245]$. difficult to compute [209] even though recent LES [229-233] allow confidence in this modeling strategy for more complex geometries. The main specificities of swirl confined flows are illustrated on Fig. 2. The two main non-dimensional numbers controlling simple swirled flows are the Swirl number, $S$ and the Reynolds number, $R e$ :

$$
\begin{aligned}
& S=\frac{G_{\phi}}{R G_{x}}=\frac{\int_{0}^{R} U W r^{2} \mathrm{~d} r}{R \int_{0}^{R} U^{2} r \mathrm{~d} r}, \\
& R e=\frac{U_{0} R}{\nu} .
\end{aligned}
$$

$S$ measures the ratio of the axial flux of the swirl momentum, $G_{\phi}$ [ $\left.\mathrm{kg} \mathrm{m}^{2} \mathrm{~s}^{-2}\right]$, to the axial flux of the axial momentum, $G_{x}\left[\mathrm{~kg} \mathrm{~m}^{2} \mathrm{~s}^{-2}\right]$ multiplied by a characteristic length of the swirl annulus, $R[\mathrm{~m}]$. In Eq. (10), $U[\mathrm{~m} / \mathrm{s}]$ and $W[\mathrm{~m} / \mathrm{s}]$ are the mean axial and tangential velocities measured in a plane usually located at the exit of the swirl generator. The Reynolds number is defined using the bulk axial velocity, $U_{0}$, the swirler annulus radius, $R$, and the fluid kinematic viscosity, $\nu$. Other definitions are possible for the swirl number and originate from purely geometrical considerations [215]. For example, the geometrical swirl,

$S_{g}=\frac{W_{0}}{U_{0}}$

is often encountered.

Depending on $R e$ and $S$, the following mechanisms and structures appear:

- Inner Recirculating Zone (IRZ): This region is created by the intense swirl. Usually located right along the axis of the swirler,

a

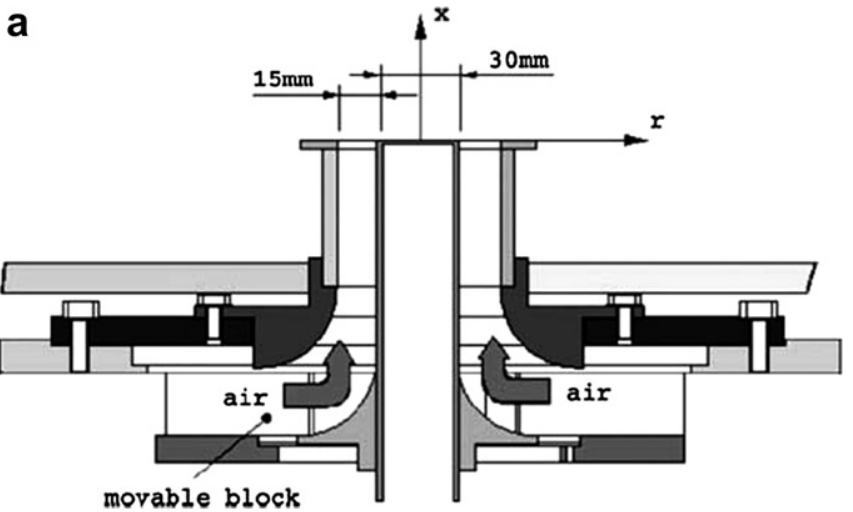

b

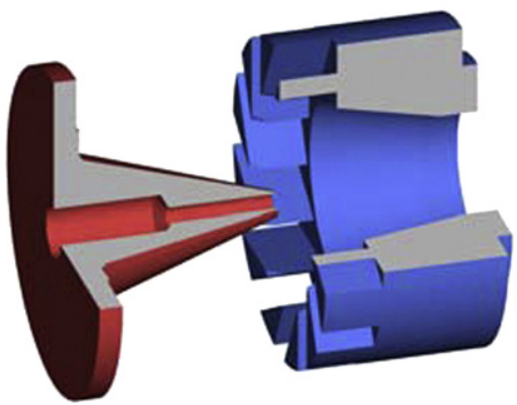

Fig. 5. Geometrical setup of (a) the TECFLAM swirler combustor and (b) TIMECOP-AE swirler used to validate LES [239-241]. 
this recirculation bubble appears for large values of $S$ (typically above 0.6). It results from the radial pressure gradient generated by the guided rotating flow (large tangential velocity component of the flow) and the flow expansion through a nozzle at the chamber inlet: the radial pressure gradient and axial velocity components suddenly decay producing a negative axial pressure gradient and a reverse flow or IRZ $[208,222-228]$.

- Corner Recirculating Zones (CRZ): In confined configurations, the sudden expansion of the flow at the chamber inlet is partly controlled by flow recirculating bubbles present at the outer edges of the dump [208,224,234].

- Processing Vortex Core (PVC) and Vortex Breakdown (VB): Under specific conditions (still not clearly mastered) the central vortex core present in the inner parts of the swirler or the IRZ becomes unstable giving rise to the PVC [224]. This destabilization can induce oscillations of the IRZ in the axial and azimuthal directions. The PVC believed to be at the origin of such oscillations coincides with a vorticity tube of helical shape located at the outer rim of the IRZ. This thin vortex tube has a helicoidal shape can be co- or counter-rotative to swirl, Fig. 3. It then can turn around the swirler axis in the swirl or opposite direction. In some cases several vortex tubes may coexist at the same time [235-237]. Finally, this specific structure is highly dependent of the CRZ and IRZ interactions [238], which are controlled by the details of the swirler and dump configurations.

LES flow predictions and validations on unconfined swirled configurations provide an evaluation of LES codes on flows typical of real burners (Table 4). A typical experiment proposed for this specific purpose is the Sandia burner described on Fig. 4 and investigated numerically by $[229,232,233]$ in its non-reacting operation. Other swirl injector systems [239-241], Fig. 5, have
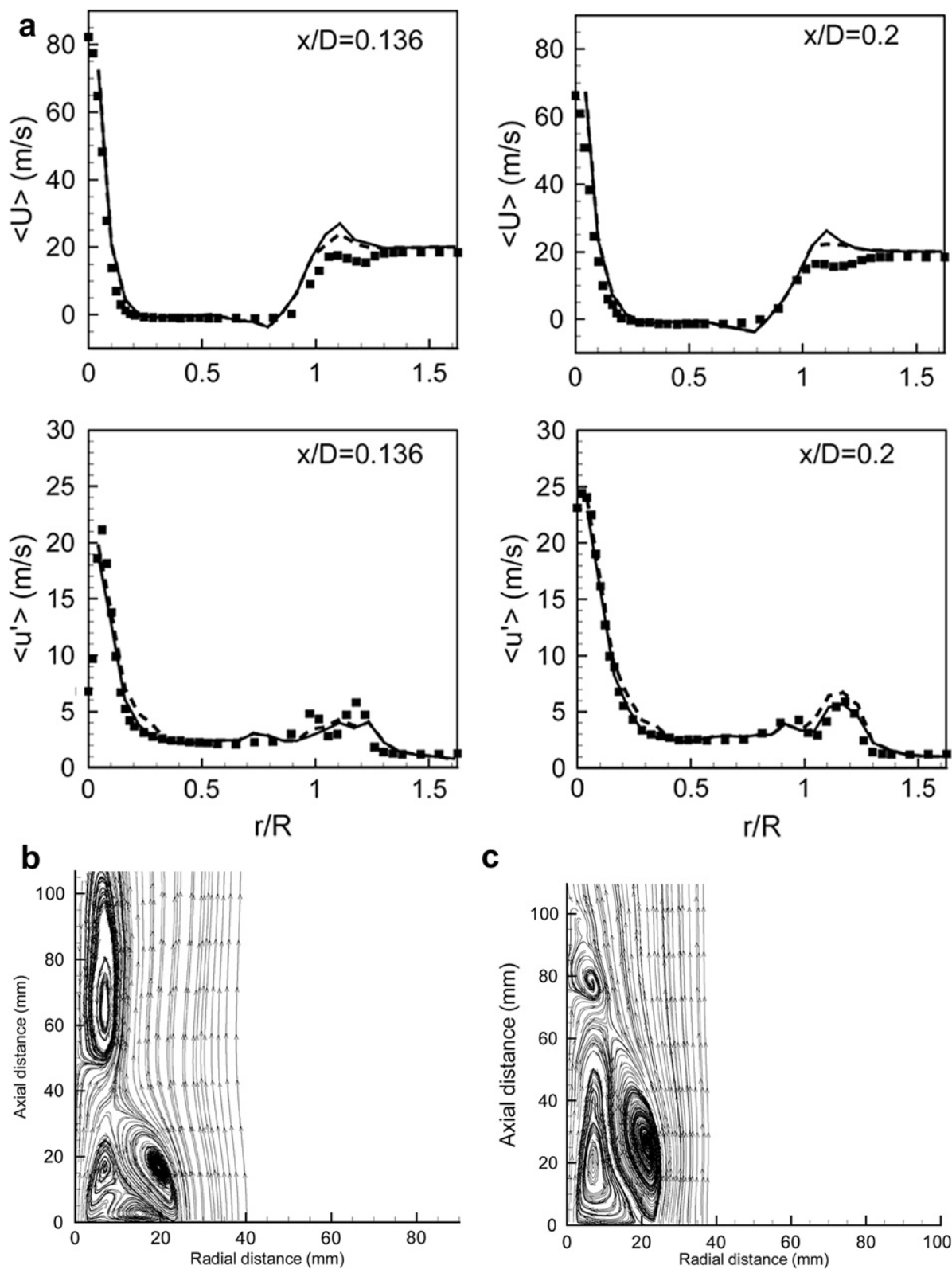

Fig. 6. Mean and RMS profiles as well as flow streamlines obtained by LES for a bluff-body swirl flow, Fig. 4: (a) - 1 million grid point LES, - - 1.44 million grid point LES, - experimental measurements; (b) high swirl and (c) moderate swirl numbers [232]. 
been also investigated numerically [230,242-244] with the same observations as the one produced below.

\subsubsection{Predictions of the mean statistical flow features}

For large swirl number and moderate swirl Reynolds number flows [215,245], LES of the configuration illustrated on Fig. 4 is found to be quite insensitive to grid resolution and SGS modeling, Fig. 6(a). At moderate swirl numbers, near Vortex Breakdown $(\mathrm{VB})$ : i.e. $S \approx 0.5$, predictions are more sensitive but remain interesting and encouraging, Fig. 6(b).SGS dynamic procedure and grid resolution may be of importance in such critical flow conditions. Inflow conditions are also suspected to be of primary importance and should at least reproduce the unsteady effect of a turbulent flow entering the computational domain. LES still remain the only current modeling strategy that correctly predicts mean statistical (i.e. mean and Root Mean Square (RMS)) flow features in strongly swirled flows.

\subsubsection{Predictions of the unsteadiness of swirl flow features}

The unsteady structures characteristic of swirl flows control the fuel and air mixing process and the interactions between the flame and the flow. These unsteady characteristics are much less investigated or validated because of the difficulty in properly characterizing such motions experimentally or numerically. The PVC is known to be weakly dependent of Re and its frequency $f$ can be expressed in terms of a Strouhal number, $f D e / U_{0}$ where De stands for the swirler exit diameter [228]. Evaluations of the LES issued PVC's [239,240] and their Strouhal numbers for the cold flow configuration of Fig. 5(a) are overall in agreement with
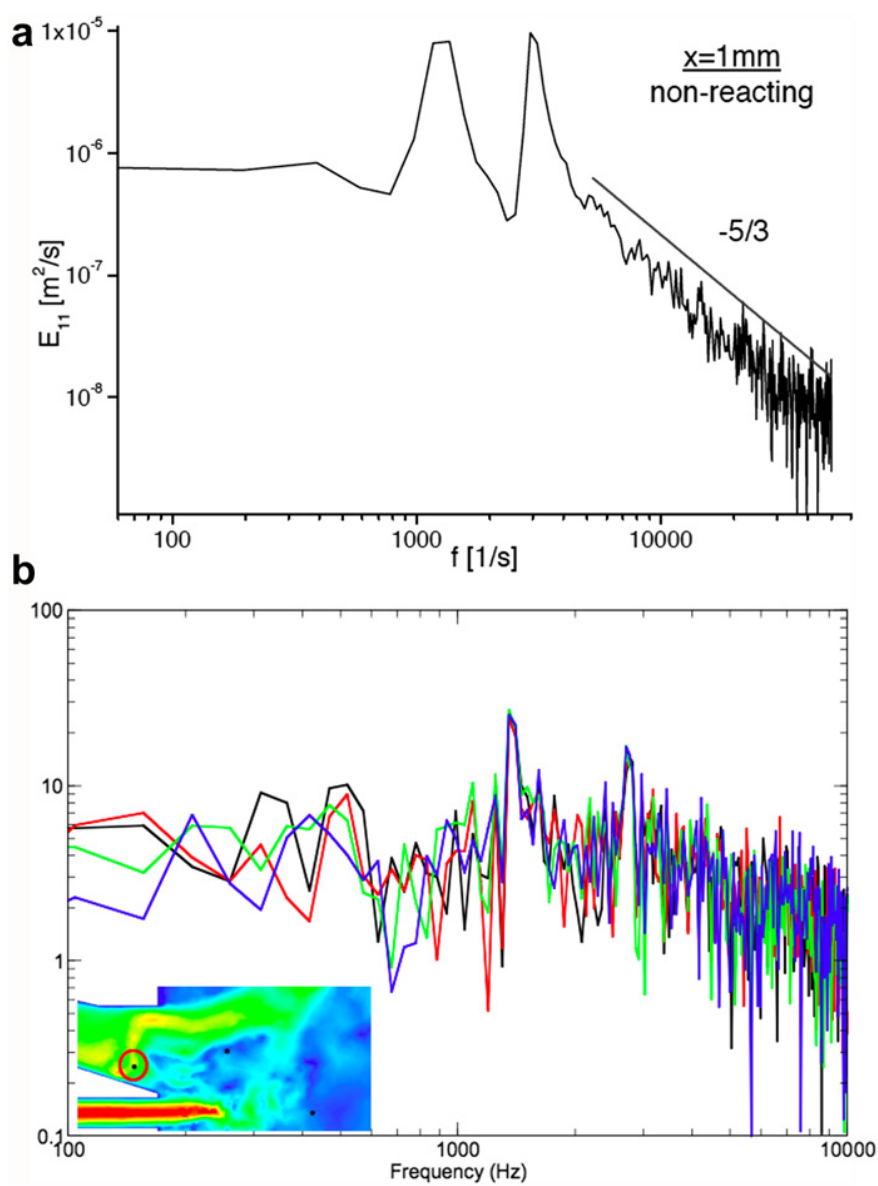

Fig. 7. Spectral analysis of the swirl flow motions in the TECFLAM swirler combustor [239-241]: (a) experimental measurements and (b) corresponding LES spectra. experimental findings [241,246-250], Fig. 7; typical results report approximately $10 \%$ errors when comparing LES PVC frequencies with experimental measurements.

For these well documented geometries [215,239,240,245, 251-253], LES predictions are encouraging, Figs. 6 and 7. In configurations where $S$ is above the critical swirl number of $0.5-0.6$ where VB is expected $[238,254,255]$, results are very satisfactory (cf. Sandia configuration of Fig. 6). In most reacting systems, the fuel injection location within the swirler is of importance not only because it determines mixing, but also because of the interaction and potential flow topology change they may have on the swirling flow. Jet/IRZ interaction is often present and impacts the evolution of the PVC and even the IRZ itself as discussed by [228] (cf. Fig. 6 for a typical example).

\subsection{Reacting flow validations}

LES of reacting flows is a relatively new research topic which only emerged in the early nineties and became a focus point of CFD research in the late nineties. This contrasts with research on 'pure' LES (i.e. without reaction) that appeared in the sixties in the weather-forecast community $[83,90]$. Many reasons explain these differences. The first one probably originates from the turbulent combustion community itself which dedicated a lot of effort in implementing new combustion models for RANS and naturally lags the turbulent community. Second, computer power and the emergence of highly efficient machines and algorithms only recently allowed to address even simple laboratory-scale configurations which was a necessary step for the turbulent reacting LES
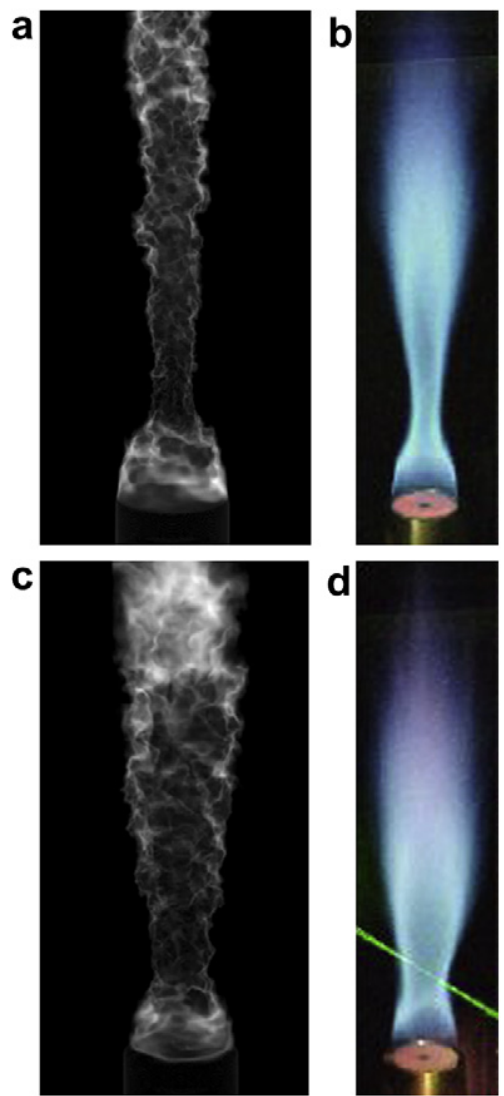

Fig. 8. Geometrical setup of a typical swirled flame for which detailed measurements are available. First row, SMH1 flame: instantaneous views of (a) Imperial College LES predictions [258] and (b) the associated experiment [215]. Second row, SMH2 flame: instantaneous views of (c) Imperial College LES predictions [258] and (b) the associated experiment [216]. 
concept to be validated. Finally, very few theories or mathematical models are available for turbulent reacting flows for conceptual validations. This is clearly not the case for turbulent non-reacting flows. In parallel to these efforts, new flame modeling concepts appeared and the turbulence as well as the turbulent combustion communities started to recognize the role of the most energetic flow structures in CFD. All these developments are transcribed in the TNF workshop series [256] or Combustion Symposium series [257] which mainly addressed RANS modeling validations until the 1990s and now focus almost exclusively on LES.

The following discussion focuses on some of the recent contributions and efforts in the field of reacting LES in swirled configurations. First, statistically stationary unconfined and confined

a

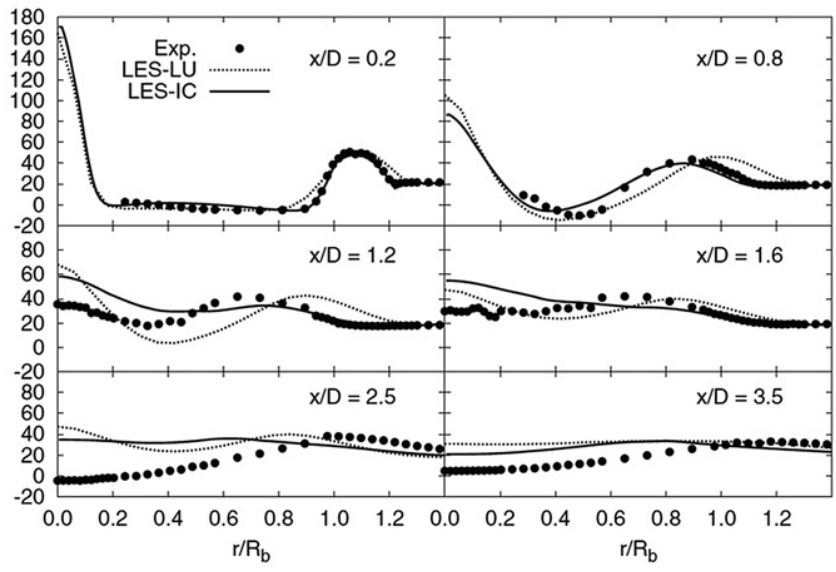

simple configurations are reviewed followed by a discussion on recent leading-edge LES applications to illustrate the possibilities of massively parallel architectures. Finally, two specific research subjects benefiting from these recent LES developments are discussed: (1) thermo-acoustic instabilities often encountered in real gas turbine engines and (2) transient phenomena (ignition, extinction sequences...).

\subsubsection{Statistically stationary flow conditions}

Many LES contributions mainly aim at validating SGS turbulent combustion models ( $c f$. the series of TNF workshop proceedings and comments on the matter). Swirled flames have been computed only recently, Fig. 8 (predictions for the Sandia burner of Fig. 4), and
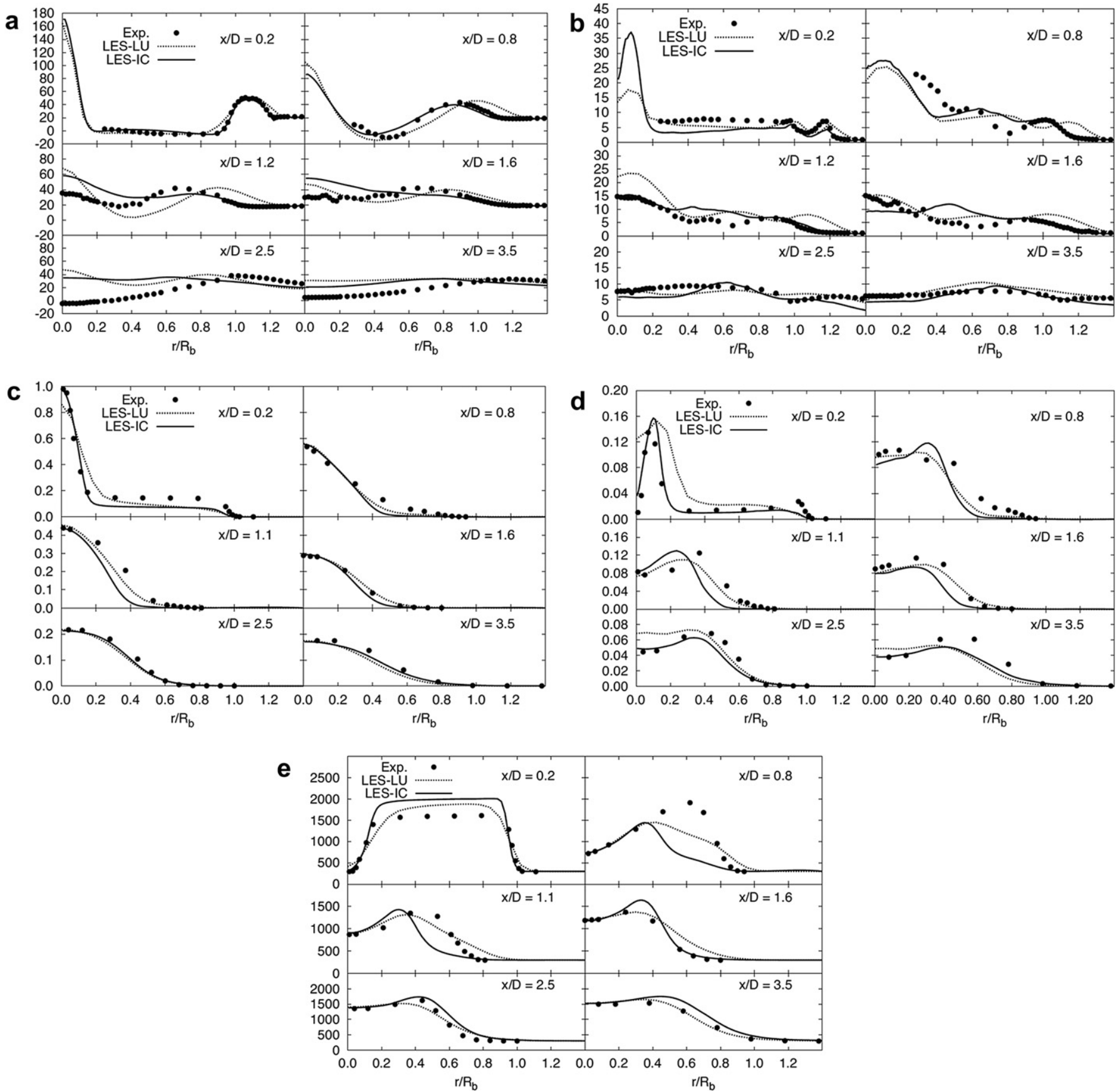

Fig. 9. Comparisons of the numerical predictions [258] with experimental data [215] for SMH1: (a) mean axial velocity profiles, (b) mean mixture fraction and their respective RMS (b) and (d) along with (e) the mean temperature profiles. 
a

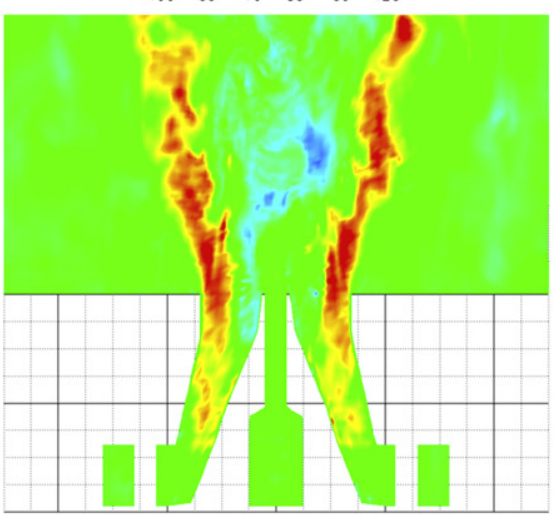

b

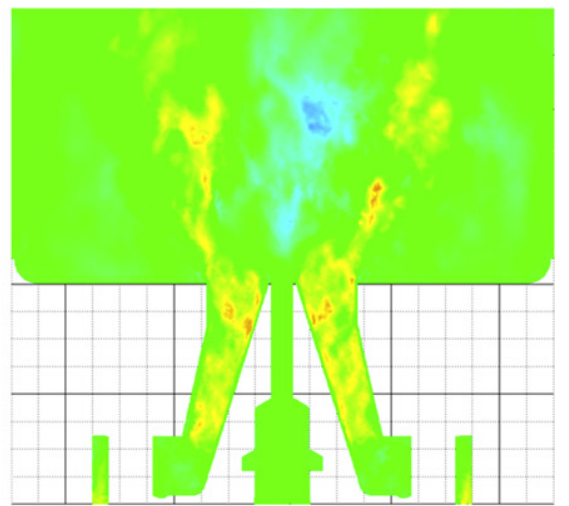

C - CERFACS - - TUD $\quad 0$ Expe. (Transverse direction) $\square$ Expe. (Cross-stream direction)
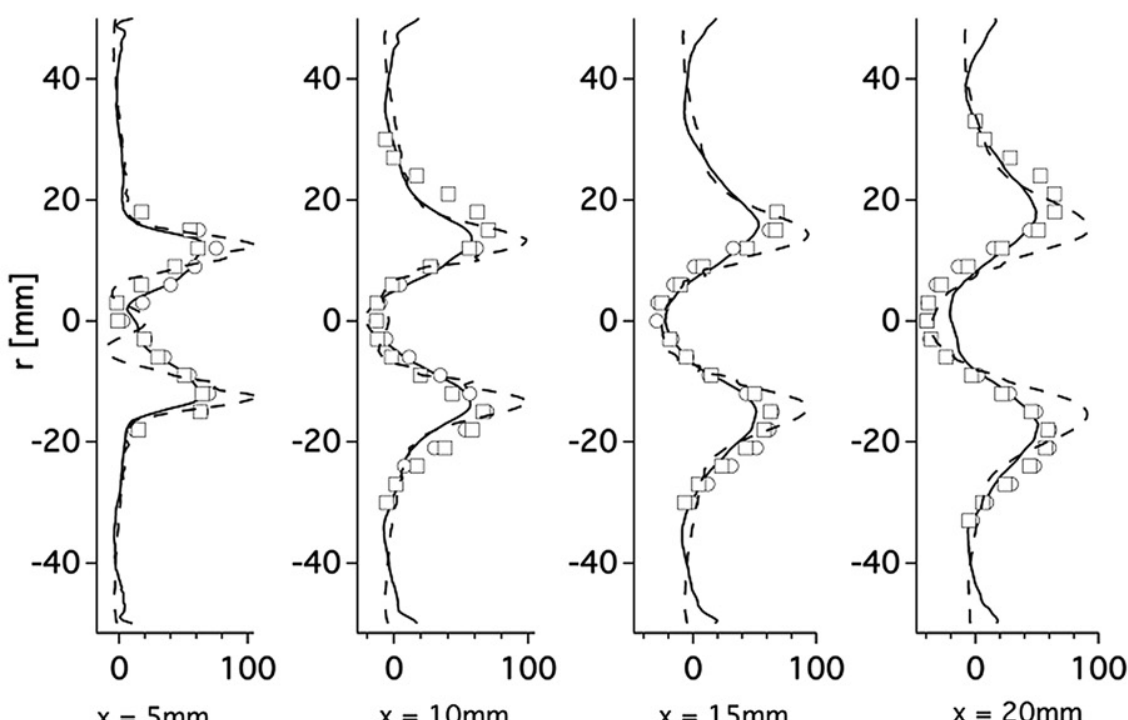

$x=5 \mathrm{~mm}$

$\mathrm{x}=10 \mathrm{~mm}$

$x=15 \mathrm{~mm}$

$x=20 \mathrm{~mm}$

d - CERFACS - - TUD $\quad 0$ Expe. (Transverse direction) $\square$ Expe. (Cross-stream direction)
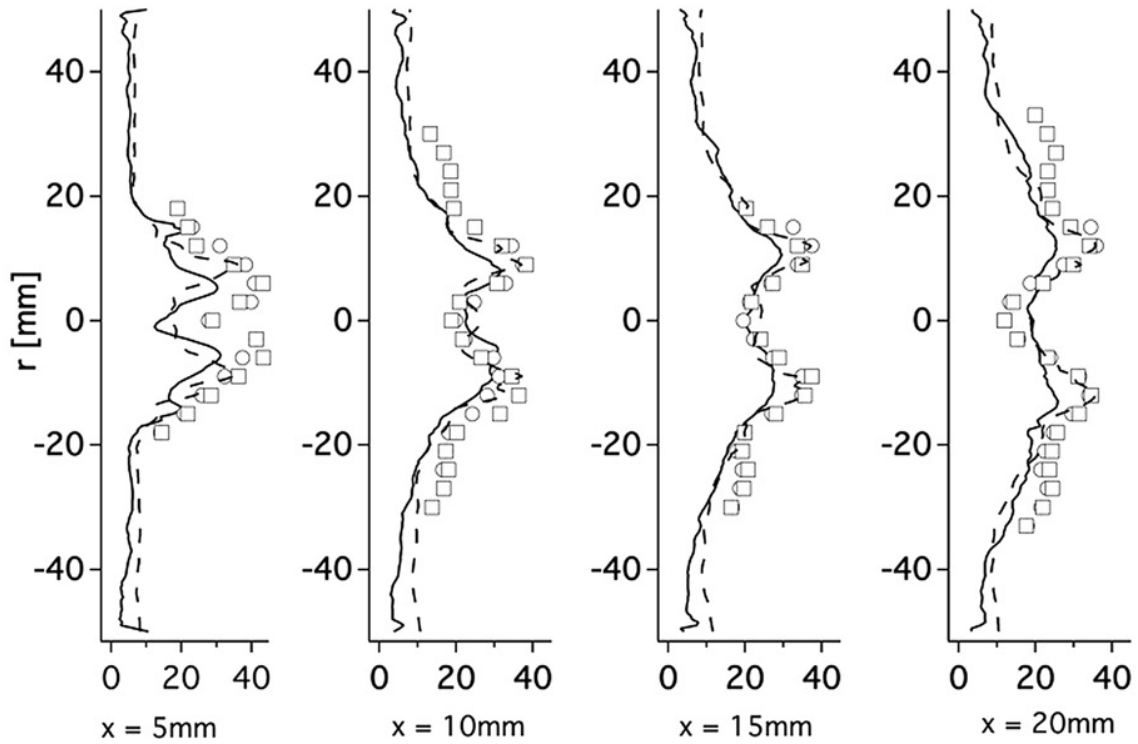

Fig. 10. Instantaneous views of the axial velocity component: (a) TUD, (b) CERFACS. Temporal averages of the two LES's are compared to experimental measures for (c) the mean axial velocity component and (d) its RMS at four axial locations within the chamber (courtesy of P. Pantangi, A. Sadiki, M. Haege and A. Dreizler from TUD University, Germany). 

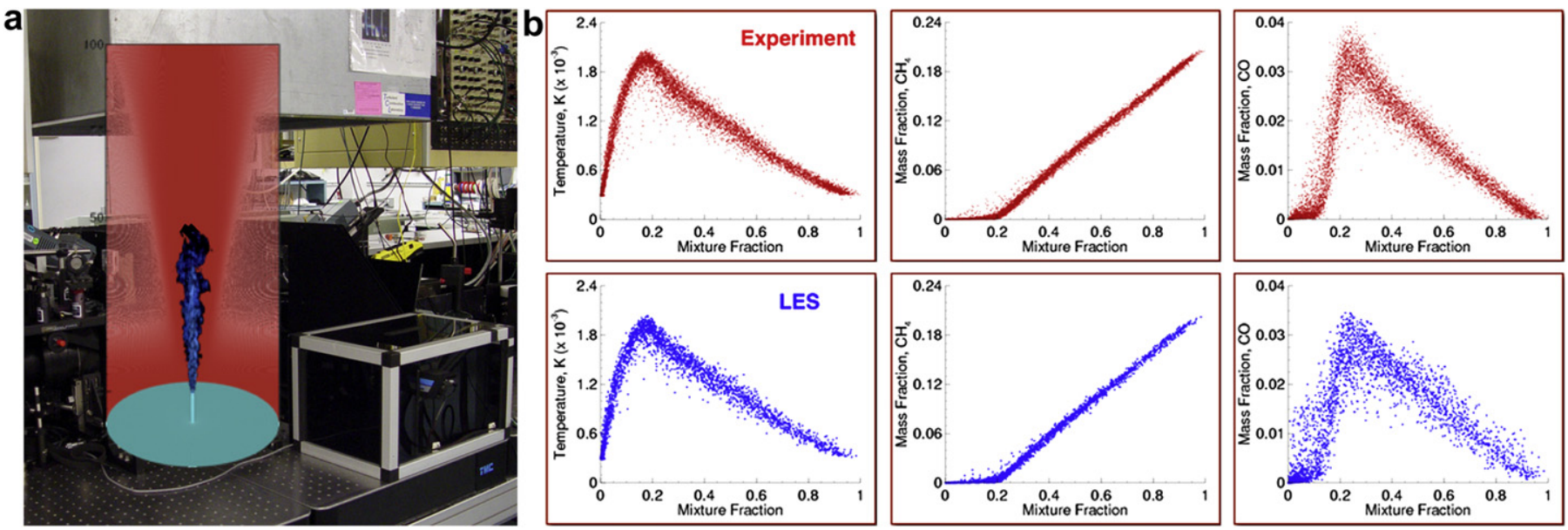

Fig. 11. (a) DLR-A flame configuration and highly resolved LES mesh along with (b) a comparisons of scatter plots obtained by measurements (red) and LES (blue) [273]. (For interpretation of the references to color in this figure legend, the reader is referred to the web version of this article.)

most of the effort has been concentrated on jet flames. A list of the identified productions for laboratory swirled flames is given in Table 4 . These studies generally demonstrate the superiority of LES for turbulent reacting flows. The main reason originates in the natural unsteady nature of the governing LES equations which dynamically reproduce the interactions that control mixing and turbulence/flame interactions over a wide range of applications. These laboratory tests have however a major limitation to qualify SGS sub-models for mixing and turbulent flame interactions: their Reynolds number is low inducing a significant overlap of the inertial and dissipative scales thereby violating the scale separation hypothesis needed for most SGS closures. Conclusions are thus difficult to extrapolate to real gas turbine flows where the flow Reynolds number is much higher. Fuels are also much more energetic (thinner flame fronts) and grid resolutions much lighter. All of these issues have been identified and highlighted in Section 2.2.2. The difficulty reduces in discriminating turbulent combustion models based on reliable quality criteria in the context of mixing and reaction. This last subject still remains an open issue despite recent contributions [200].

Recent LES publications on gaseous laboratory scale swirl flames for the Sandia burner illustrated on Fig. 4 and complementing the predictions of Fig. 8, provide first insights on the importance of modeling strategies. This example was produced jointly by researchers in England (Imperial College and Loughborough University) [258] with two low Mach number structured (in cartesian and/or cylindrical formulations) LES codes. Results are provided on Fig. 9 for the swirled experiment of $[215,216]$ operated with methane, air and hydrogen for two swirl numbers $\left(S_{g}=0.32\right.$ for SMH1 and $S_{g}=0.54$ for SMH2, respectively). Although the codes differ, they theoretically use the same LES formalism. The simulations are produced on different grids and slightly different boundary conditions. SGS velocity closures rely on dynamic closures [24] with numerical regularization for unphysical model coefficients (clipping). Turbulent combustion modeling relies on single or multiple flamelet approaches, Eq. (9). Chemical terms are obtained from two chemistry models with variable or constant strain rates and a subgrid scalar variance, $\tilde{Z} Z-\tilde{Z} \tilde{Z}$, is coupled to a presumed Beta PDF. Depending on the LES method available in each code, the scalar variance is closed either by use of [259] or [260].

Mean velocity, mixture fraction and temperature predictions for SMH1 are compared to experimental data on Fig. 9. When available RMS profiles are added. Similar results for SMH2 are also available
[258]. Overall, both LES codes and strategies provide similar behaviors. Grid sensitivity and inflow boundary specifications are specifically highlighted underlying the difficulty of clearly differentiating one modeling strategy compared to another one (see above discussion on boundary condition effects). However, both approaches provide very satisfactory predictions of the mean and RMS flow fields irrespectively of the flow swirl number. Mixing is well predicted for both experimental conditions. Mean temperature profiles are consistent with experiments.

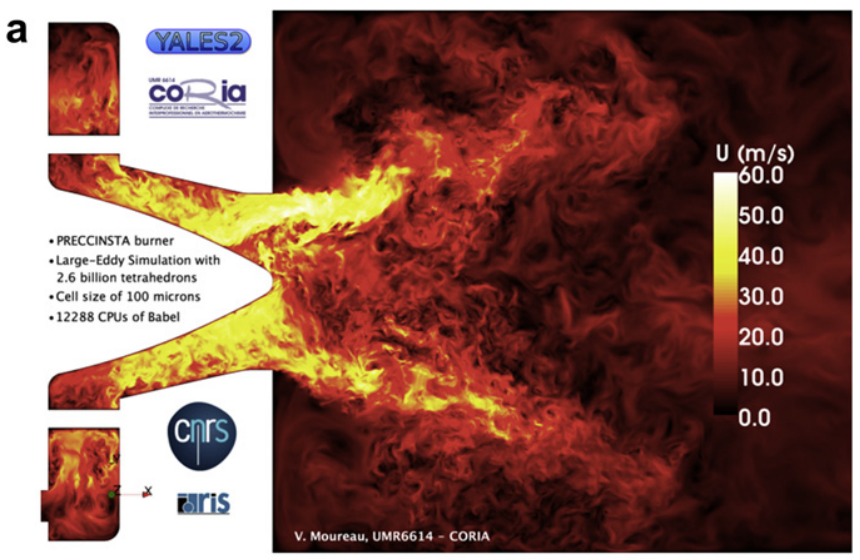

b

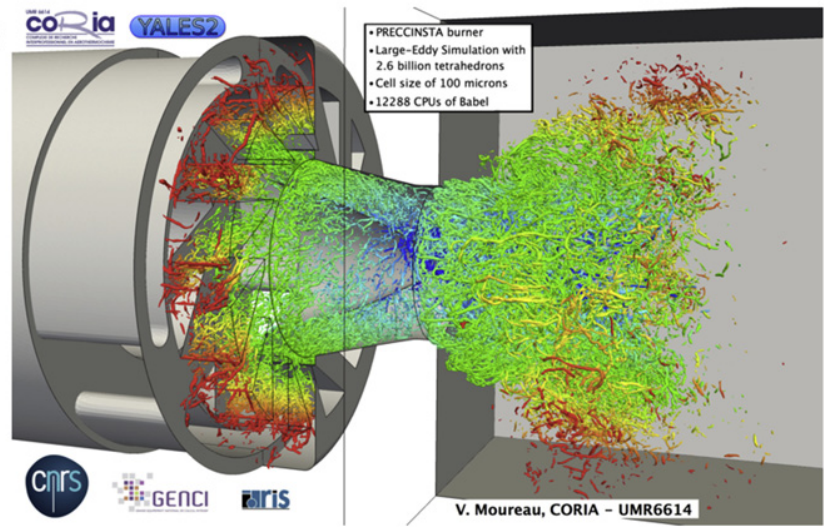

Fig. 12. Massively parallel LES predictions of the Precinsta burner: (a) instantaneous velocity field and (b) turbulent structures [205]. 

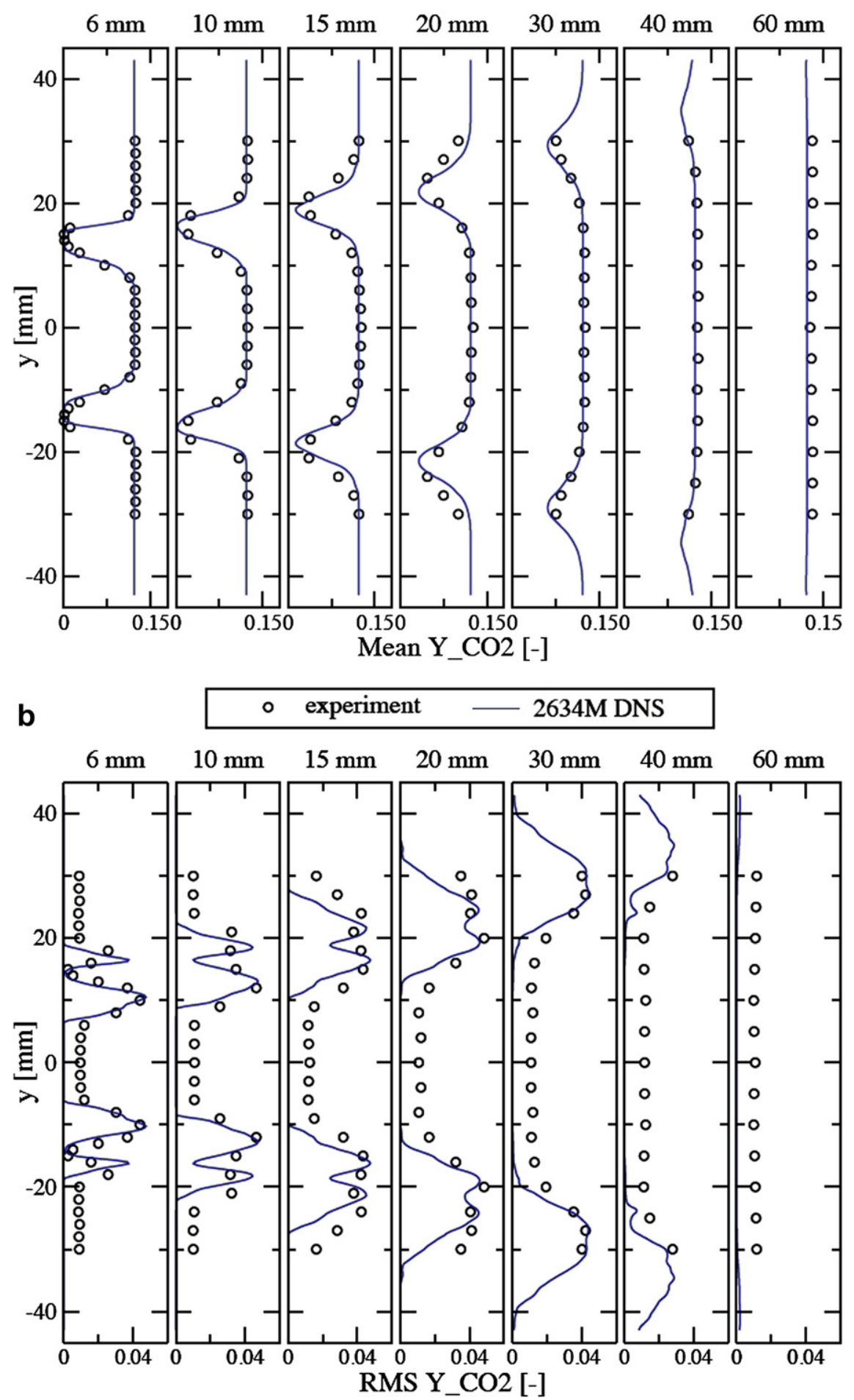

Fig. 13. Mean profiles of (a) the $\mathrm{CO}_{2}$ mass fraction and (b) its RMS obtained by LES and in the experiment at different stations within the combustor

Similar validations against laboratory scale burners with more complex geometries start to appear. Such a comparison has recently been produced within the context of the TIMECOP AE European project ${ }^{1}$ involving major European aeronautical engine

1 TIMECOP AE stands for Toward Innovative Methods for Combustion Prediction in Aero-Engines, FP6-2005-Aero-1. manufacturers. This specific study follows the MOLECULES European project ${ }^{2}$ where the same injector was studied but operated with gaseous methane [240,241]. For the case discussed here, pure gaseous kerosene is injected separately from the swirled air stream

2 MOLECULES stands for Modeling of Low Emissions Combustors using Large Eddy Simulations, GRD1-2000-2522. 
and the rig is operated at different mean pressures. Details on the swirler geometry are visible on Fig. 5(b). Both computations include the fuel injection system: i.e. the swirler veins and the fuel axial pipe, Fig. 10(a) \& (b), to avoid specifying inflow conditions which may impact the predictions. Here again mesh resolution, numerics and formalisms differ. The flow solver from Technische Universität Darmstadt (TUD) is a low Mach number, second order accurate in time and space, multi-block solver. CERFACS's code is third order in time and space, explicit and fully compressible. Turbulent combustion modeling also differ. The latter relies on tabulated chemistry and a conserved scalar [144] approach while the former uses a reduced two-step mechanism [261] coupled to the Dynamic Thickened Flame model [262]. The main outcome is a weak but observable difference in exit swirler axial velocity and RMS profiles, Fig. 10(c) \& (d), obtained with the two codes. The main reason for such findings is the relative difference in axial momentum flux at the fuel jet exit predicted by each simulations (different jet profiles). These small flow differences impose changes in IRZ topologies which in turns impact the flame stabilization mechanism and localization. In fact one LES prediction produces a lifted flame when the other predicts a flame anchored slightly inside the swirler. Of course turbulent combustion modeling and most likely chemistry is involved in such stabilization processes. However the importance is not clear. Despite these observations, mean and RMS flow predictions agree with experimental data and uncertainties do not exceed $10 \%$ if compared to experimental findings which also contain measurement errors. Note that direct views of the operating burner could not clearly discriminate between a lifted or anchored flame.

An open question which seems relevant in light of the previous comparison is the actual IRZ and PVC dynamics in confined and complex reacting flows. No clear experimental assessment of PVC behavior in combusting conditions is currently available although experimental investigations specifically point to such issues $[240,253,263]$. Certain LES results confirm the presence of a PVC in cold flow conditions and observe its presence or disappearance in reacting flows. Such a structure is of critical importance especially for complex systems where fuel injection is usually located in the near region of the PVC [264]. The PVC will play a role in the flame stabilization process or flow transition from one operating condition to another. This is an additional difficulty to qualify LES in an industrial context since such behaviors may be amplified or damped by modeling and discretization errors. At least the question emerges due to the potential benefit of simulating fully unsteady features by LES which is not possible with RANS.

\subsubsection{Leadership-class LES modeling and predictions}

In recent years, the advent of massively parallel machines offering PetaFlops (one million billions of floating point operations, $10^{15}$, per second) $[206,265]$ or projections for ExaFlops $\left(10^{18}\right)$ capabilities in 2020. Such new horizon and machines infer a new impetus to code developers and new coding strategies or data management schemes to better benefit from the added computing power. The net result is the emergence of new LES codes able to manage efficiently hundred thousand and even billion points LES. With such capabilities new modeling constraints appear and help understanding or assessing each model contribution in specific and well mastered circumstances by seriously reducing the impact of numerics for example. This recent environment yields new types of LES results that are presented here. Issues pertaining to the nearly fully resolved fields are also illustrated.

3.3.2.1. Highly resolved LES predictions. This brute-force method has produced quite successful results for the DLR-A flame [266-268] and the PRECCINSTA burner [269-271]. Fig. 11 presents (a) the DLR-A set-up along with (b) scatter plots of temperature, methane and $\mathrm{CO}$ mass fractions as functions of the mixture fraction space, $Z$, at given axial stations in the jet obtained by measurements and LES $[272,273]$. Predictions and measurements are in excellent
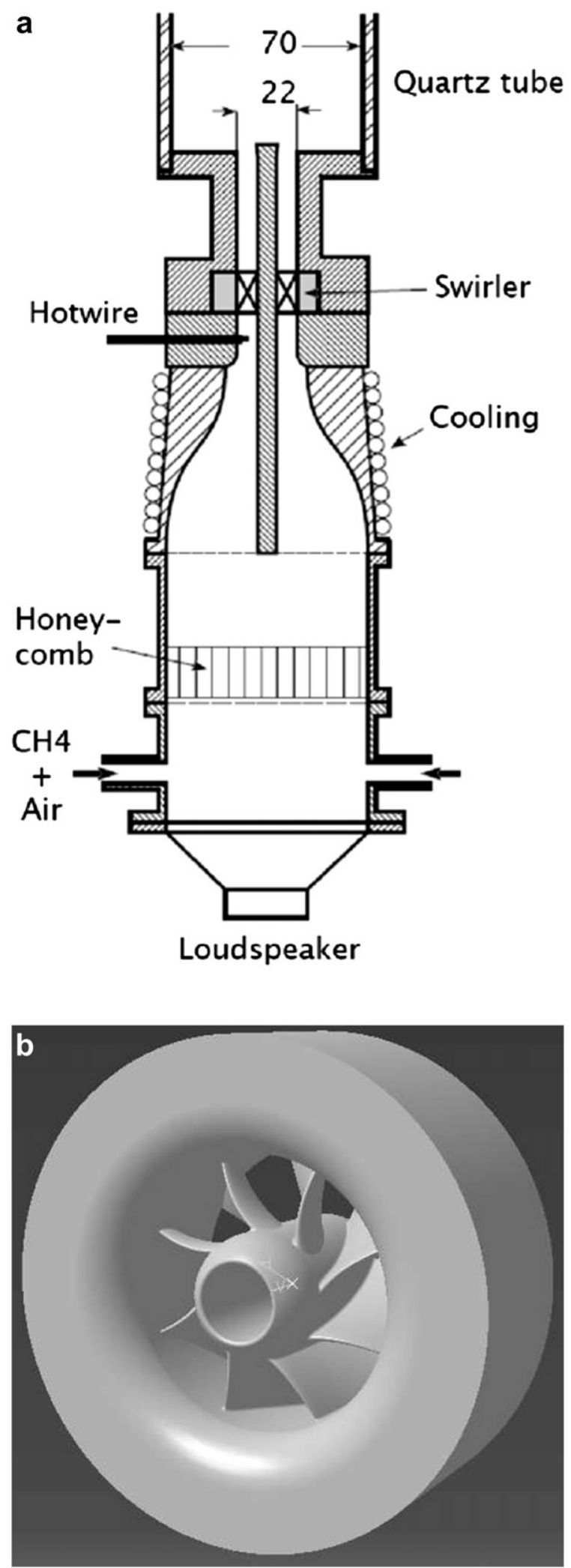

Fig. 14. Views of (a) the experimental burner and (b) swirler used to determined FTF/ FDF's experimentally and numerically [302] 


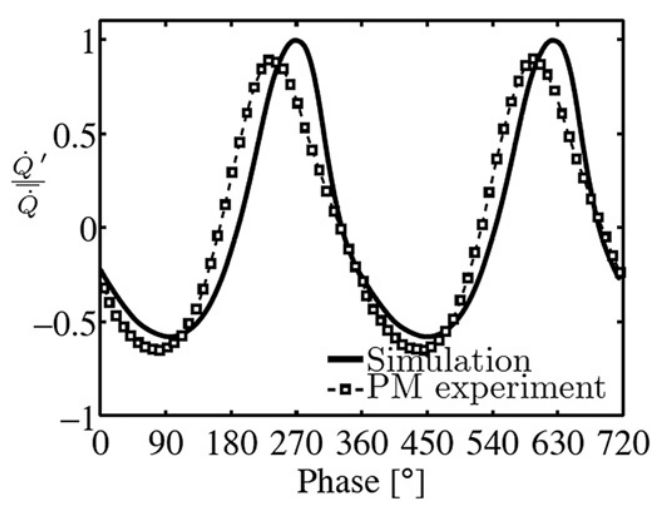

Fig. 15. Temporal evolution of the heat release rate fluctuation obtained by LES and experimentally [302] for the above configuration, Fig. 14.
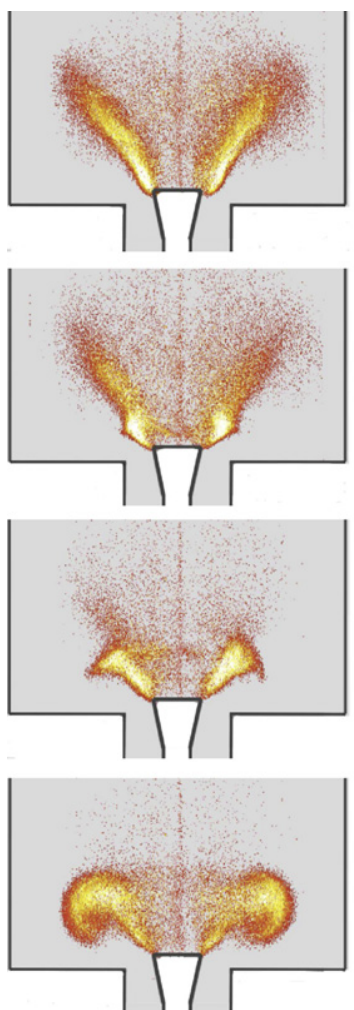

$120^{\circ}$

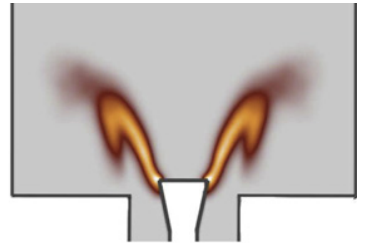

$0^{\circ}$

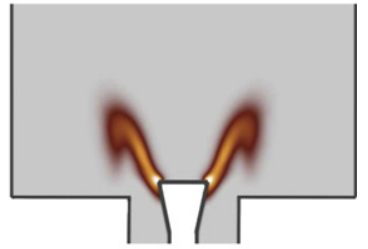

$60^{\circ}$

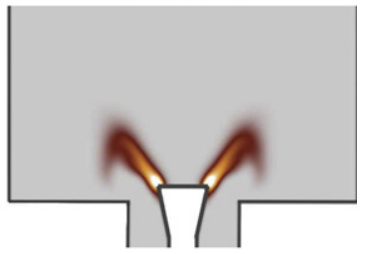

$180^{\circ}$
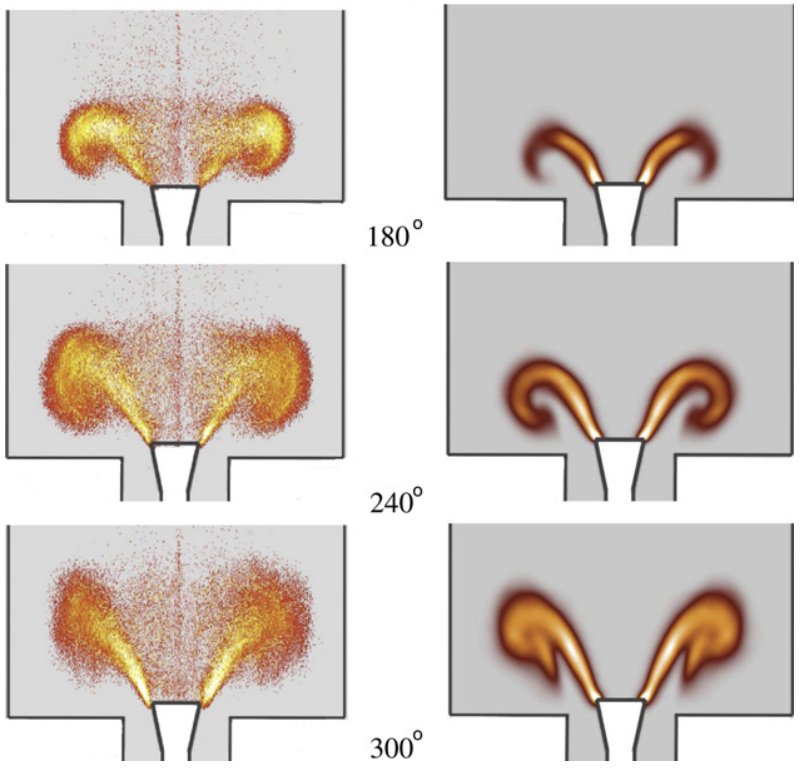

$240^{\circ}$

$300^{\circ}$

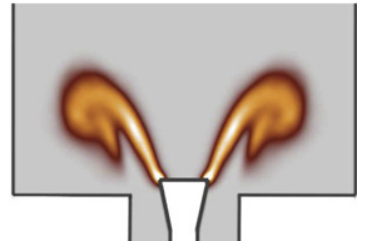

Fig. 16. Direct comparisons of experimental observations (left column) and LES predictions (right column) for a swirl stabilized premixed flame subject to an external acoustic forcing. All snapshots are taken at equal phase angles and allow to retrieve leading mechanisms governing the FDF [302]. agreement. This is also confirmed for mean and RMS velocity profiles at multiple axial locations [273]. Higher order quantities that are usually required and of importance for higher Reynolds number flames (here the reported value is $R e \approx 15,000$ ) can be probed accurately in the simulation and in the experiment [273] to validate the modeling strategy. Conclusions derived from these analyses are useful but only constitute a first step toward higher order and model validations for real industrial configurations that use much more complex fuels and operate at much higher Reynolds numbers.

More complex configurations such as the PRECCINSTA burner [269-271] for which $R e=40,000$ have also been treated with such codes [190,192,205,247,274-277]. In [192,205] and although modeling hypotheses are still present, numerical predictions and mesh independence have been obtained for mean flow quantities and RMS in cold flow conditions, Fig. 12. For the stations of interest which target the IRZ, various LES grid resolutions are obtained with a highly resolved LES (claimed to be a DNS provided that modeling can be disgarded) using 2.6 billion tetrahedral cells by use of a low Mach number code relying on a fourth-order finite volume scheme. For this cold flow condition, LES converges reasonably well with first and second order mean flow statistics becoming independent of the grid resolution for 329 million or more tetrahedra. In reacting conditions, convergence can also be reached but at a higher cost: i.e. for this tool and modeling at least $\approx 450$ million cells are needed [192]. Such findings are very encouraging since they confirm that even outside the theoretical framework for which models are derived and specifically in near realistic experimental setups, convergence is accessible. The next step is to clearly assess the importance of the modeling hypotheses by a posteriori validations and identifications of the various terms

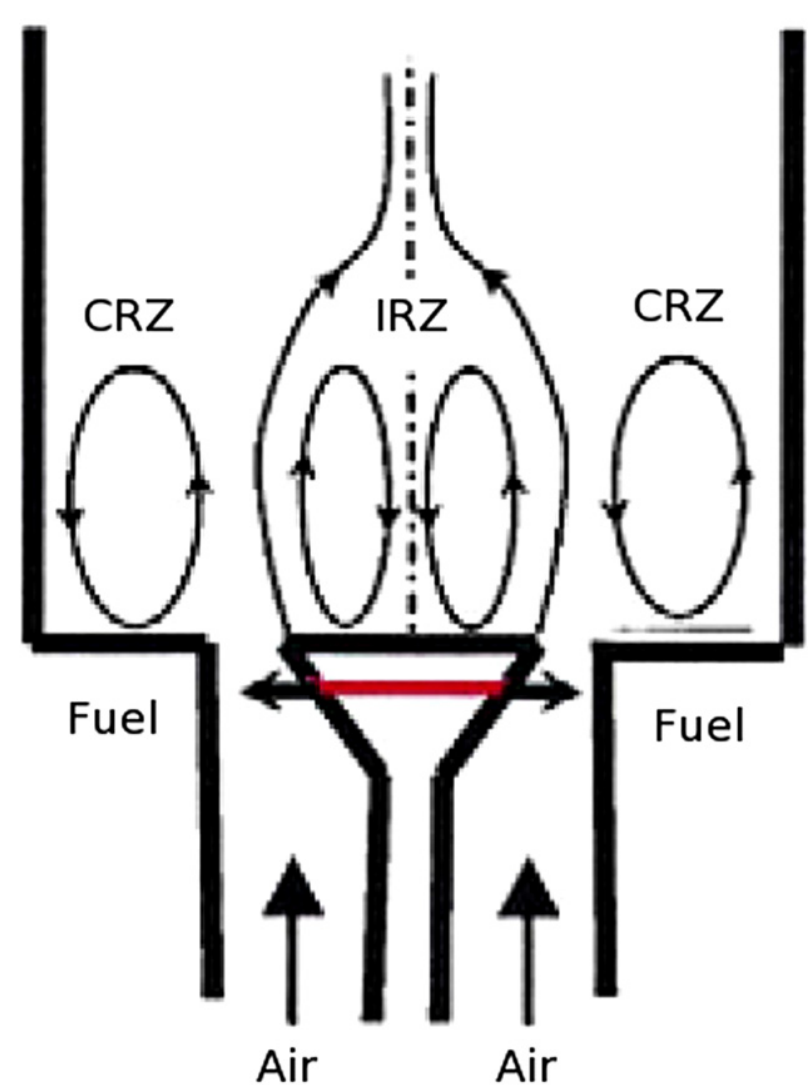

Fig. 17. Configuration to study ignition in a bluff body case [314]. 
at play in such predictions (i.e. detailed estimates of balance equations).

Comparisons of mean combustion quantities with experimental findings, Fig. 13(a), provide excellent agreement for all major species profiles. Uncertainties remain present for RMS values of species mass fractions, Fig. 13(b). Issues pertaining to the actual accuracy of the measurements for these quantities need to be precisely known. For example, the experimental measurement volume is larger than the cell size currently used in the computation. Likewise the time scales integrated and represented by both diagnostics differ: LES can only reasonably compute few flowthrough times (generally milliseconds) when measurements run over several minutes. Finally, modeling is still required in such LES. Typically, the hypothesis of a perfectly premixed burner is assumed in this work when recent experimental and numerical findings show that incomplete mixing is present in the experiment [271,277]. Tabulation is also required and specific closures valid under the purely premixed combustion are used. Another interesting question (especially from a pure industrial point of view) is: what modeling terms among the numerics, LES models, boundary conditions... provide the leading contribution to these predictions and to what level?
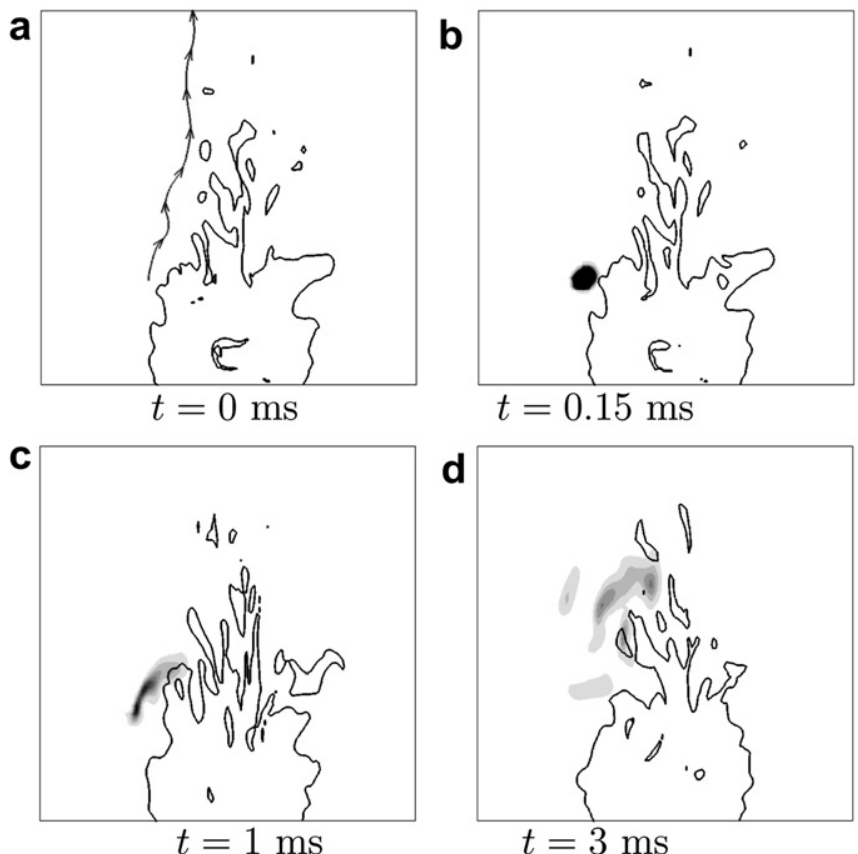

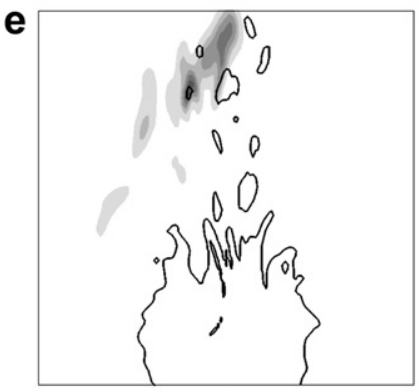

$t=5 \mathrm{~ms}$

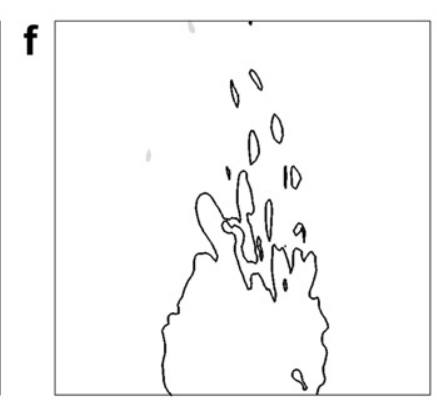

$t=10 \mathrm{~ms}$
Fig. 18. Failed ignition sequence as observed in time by LES [310] of the experimentally diagnosed configuration of [314] shown on Fig. 17. Snapshots correspond to the instantaneous field of temperature (dark iso-contours) after sparking at $t=0 \mathrm{~ms}$. Each instant also shows the isostoichiometric line.
3.3.2.2. Highly resolved LES modeling. New code capabilities conjugated with massively parallel machines offer an alternative view to the conventional turbulent combustion LES modeling strategy. Indeed, in the long term, LES (and even DNS) grid independent solutions will be applicable to some real industrial flow problems. In other words, we will simply compute most of phenomena that are today modeled. Even though this perspective is exciting and will certainly become true in the next years for simplified lab-scale burners [205,278], it remains probably a very long term option in gas turbines. First, as pointed out in [190,279], conventional approximations provided for the filtered viscous stress tensor may not be sufficient to fully recover expected flame behaviors in the context of fully resolved premixed flames [191]. LES models do not converge to DNS when the number of grid points increases because SGS models usually neglect certain effects. For example, Schmidt numbers are often assumed to be equal in LES, an assumption which may be acceptable for LES but not for DNS. Similarly many SGS models derived for premixed turbulent flows cannot capture a laminar or well resolved laminar front. Finally, technological devices present in real gas turbine combustors (effusion cooling, two-phase flows) will require modeling even on a petascale machine. a

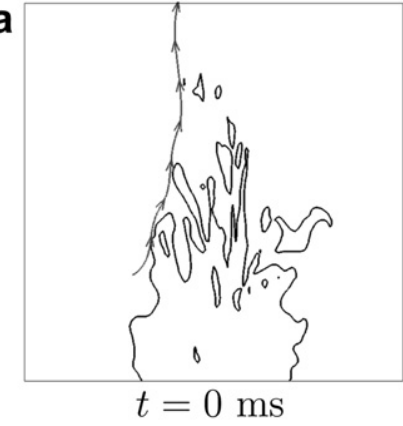

C

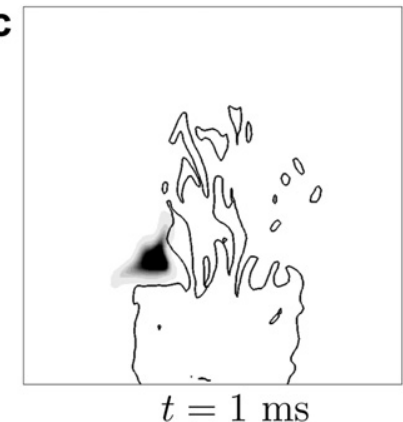

e

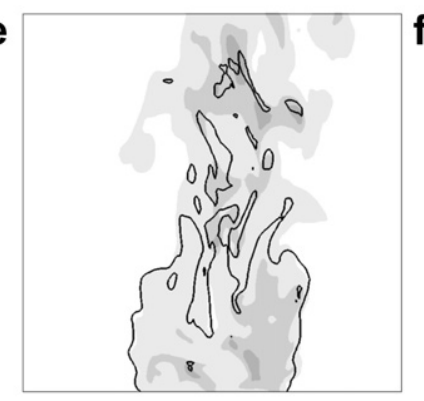

$t=20 \mathrm{~ms}$
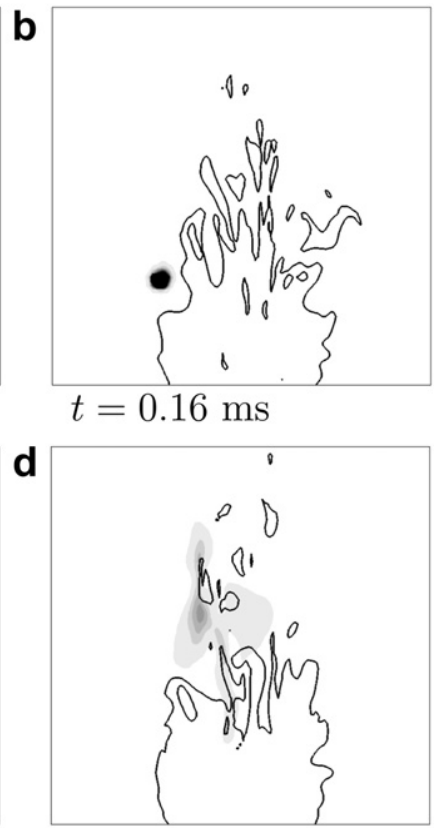

$t=3 \mathrm{~ms}$

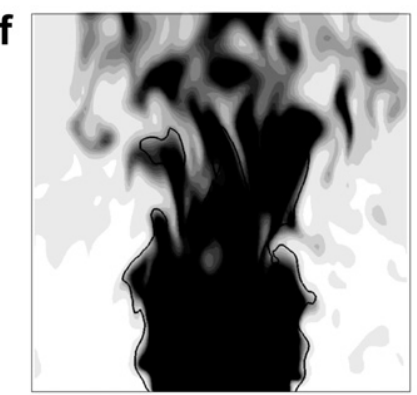

$t=45 \mathrm{~ms}$

Fig. 19. Successful ignition sequence as observed in time by LES [310] of the experimentally diagnosed configuration of [314] and shown on Fig. 17. Snapshots correspond to the instantaneous field of temperature (dark iso-contours) after sparking at $t=0 \mathrm{~ms}$. Each instant also shows the isostoichiometric line. 


\subsubsection{Thermo-acoustic instabilities}

Thermo-acoustic stability of gas turbine combustors has been the subject of intense research due to the potential constraints imposed by new regulations on pollutant emissions $[280,281]$. To meet these new objectives, conventional designs have to operate in lean premixed modes: i.e. fuel and oxidizer enter the swirler as a partially premixed gas. However such configurations are known to be prone to thermo-acoustic instabilities [31,282]. These oscillatory operating conditions must be avoided since they reduce considerably the life-time of the engine. The difficulty in predicting such physics is that the driving force involves the coupling (in phase and space) of heat release fluctuations and acoustic perturbations as evidenced by the Rayleigh criterion [31,282-288]. The combustion response to flow perturbations (acoustic or hydrodynamic) is thus the triggering mechanism.

Two numerical strategies essentially relying on LES can be adopted to investigate the thermo-acoustic response of a design. The first approach directly simulates the experimental or real geometry by use of compressible LES. The aim is to rely on the LES behavior: i.e. growth or damping of acoustic fluctuations by the computations and the eventual limit-cycle typical of a saturated thermo-acoustic operating mode. Although this approach presents some interest in real applications where no data is available, such predictions will clearly be influenced by all the parameters identified previously. It will also depend on the modeler ability to properly treat the acoustic boundary conditions of its simulation [289-293]. Extending this procedure to the entire range of conditions of a real burner is still too costly. The alternative approach is to use thermo-acoustic Helmholtz solvers and model or obtain the so called Flame Transfer Function (FTF) [286] or Flame Describing Function (FDF) [294] by use of acoustically forced LES $[293,295,296]$. This approach allows to separate the acoustic problem (which is handled by the acoustic solver) and the flame response problem (which can be computed by LES on smaller domains).

For swirled flames, the best tools to evaluate the flame response are LES or experiments [219-221,296-302]. Recent publications $[219,221,302]$ highlight the influence of swirl. In particular, the conversion of acoustic energy into vortical energy across the swirler is evidenced [302]. The main impact on the flow is a fluctuating component of the swirl number due to the convected vortical structures generated within the swirler. For such flows, the flame response not only contains the acoustic response but also a non-linear component imposed by the flow modification. A direct consequence is that the flame response depends not only on the acoustic perturbation frequency but also its amplitude. Combining LES and laser diagnostics on a laboratory swirled flame, Fig. 14, Palies et al. [302] have studied the constructive or destructive interactions of the phenomena determining the flame response.

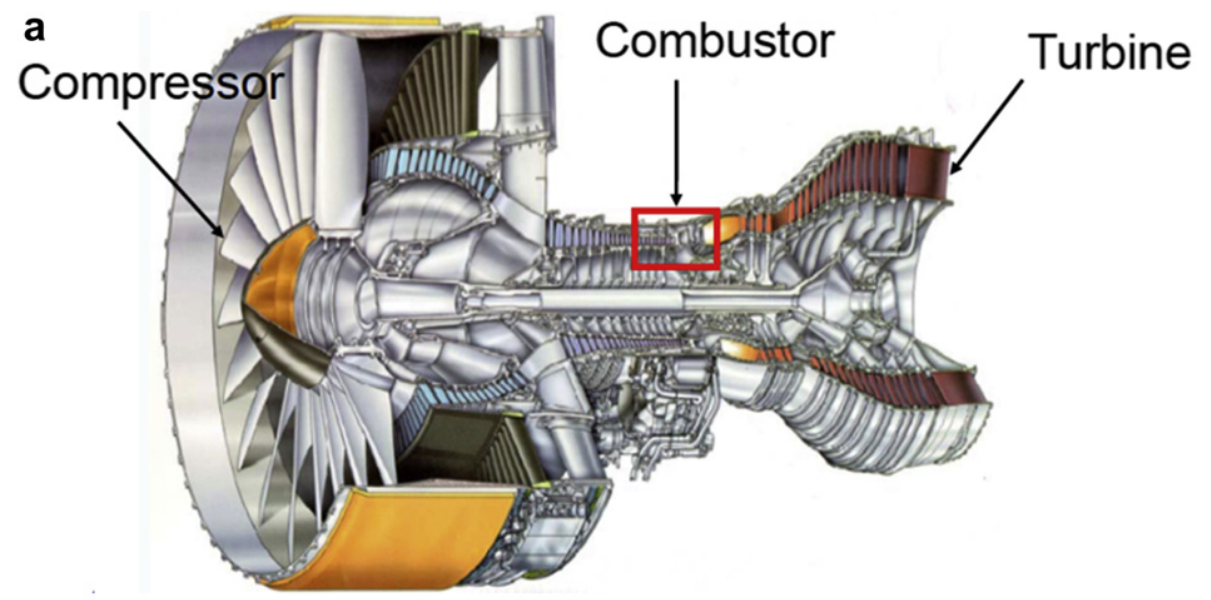

b

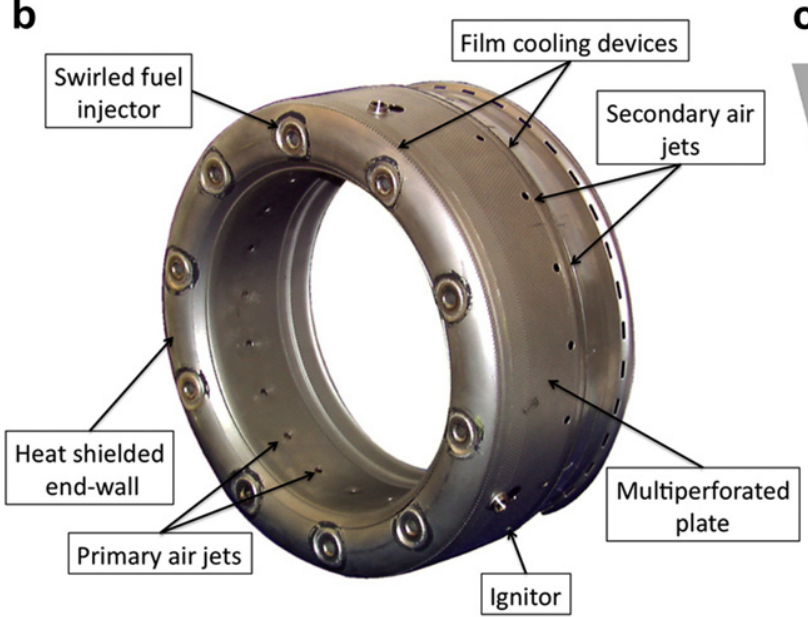

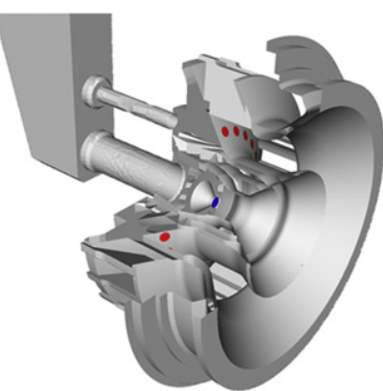

Fig. 20. Typical view of an aeronautical gas turbine engine: (a) full engine, (b) combustor flame tube and (c) a detailed view of a recent swirler design. Fuel injection points are here visualized through red dots. (For interpretation of the references to color in this figure legend, the reader is referred to the web version of this article.) 
Table 5

Tentative survey of real scale burner LES's.

\begin{tabular}{lllll}
\hline Ref. & Code & Turbulence & Combustion & Target applications \\
\hline$[316]$ & BOFFIN & Smag.-Lilly & Z, presumed PDF, flamelet & Rolls-Royce Tay engine [403,404] \\
{$[349,405]$} & AVBP & Smag. & Thicken Flame, reduced chemistry & Siemens burner \\
{$[406,318,317]$} & CDP & Dyn. Smag. & $\tilde{Z}, \tilde{c}$, presumed PDF's, complex kinetics [370] & Pratt \& Whitney combustor \\
{$[48]$} & PRECISE & Dyn. Smag. & EDB, two-step chemistry & Rolls-Royce development burner \\
{$[400]$} & LESLIE & LDKM & LEM-LES, three-step chemistry [400,407] & TAPS (GE-2) combustor [400,408] \\
{$[319,360,320]$} & AVBP & Smag. & Thicken Flame model, reduced chemistry & Helicopter combustion chamber from Turbomeca \\
{$[312]$} & BOFFIN & Dyn. Smag. & Transported PDF & Ignition sequence of a Rolls-Royce burner \\
{$[343]$} & PRECISE & Dyn. Smag. & CMC or EBU, one-step chemistry [409] & Ignition sequence of a Rolls-Royce burner \\
{$[365,366]$} & Unknown & Unknown & Unknown & Honeywell burners \\
{$[306,307]$} & AVBP & Smag. & Thicken Flame, reduced chemistry & Ignition sequence of Turbomeca burner \\
{$[355-357,342]$} & AVBP & Smag. & Thicken Flame, reduced chemistry & Helicopter combustion chamber from Turbomeca \\
{$[358]$} & Openfoam & LDKM, Mixed model & PaSR-LES [70,410], reduced chemistry & CESAR combustion chamber \\
\hline
\end{tabular}

LES are here quite successful in reproducing the experimental observations, Fig. 15. Detailed comparisons between phase averaged views of the forced burner and LES, Fig. 16, confirm the suitability of the approach (at least for the frequency of interest) to reproduce the flame response.

In parallel to these theoretical developments which now rely on the experimental diagnostics and LES, more realistic burners are treated numerically. The PRECCINSTA burner, Fig. 12, has been specifically designed for thermo-acoustic studies and preliminary LES results [247] reproduce certain unstable operating conditions. Some unstable cases could however not be recovered with fully premixed LES. In fact recent simulations [277] confirm experimental evidence that partial pre-mixing is of importance in triggering the oscillation under specific conditions $(\Phi=0.7)[271,303,304]$. Such results emphasize the need for comprehensive studies of the LES ability and modeling capabilities or sensitivity to properly understand thermo-acoustic instabilities.
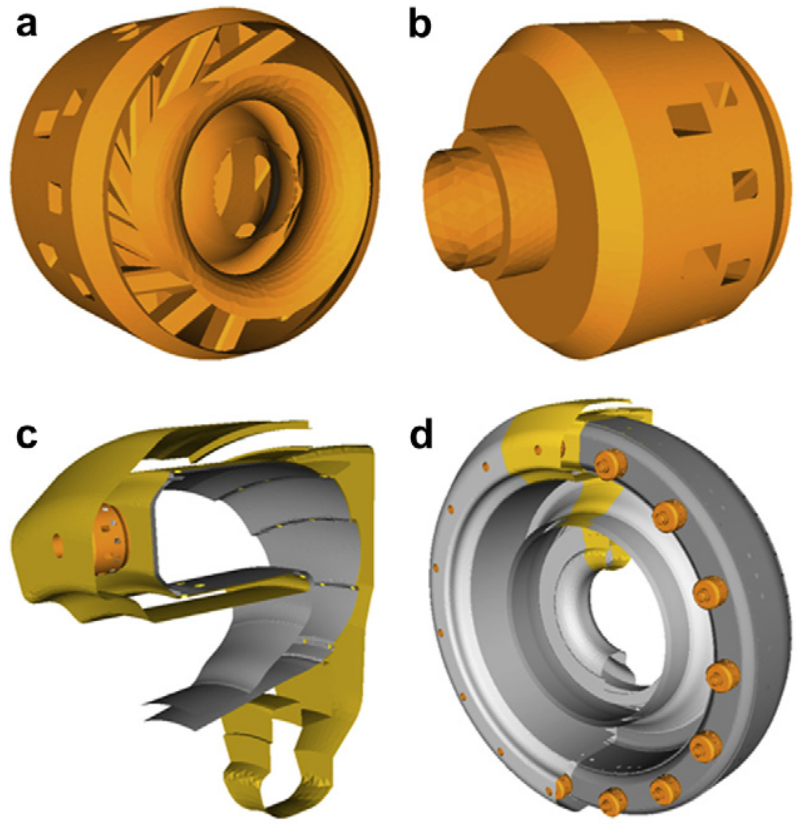

Fig. 21. Geometrical elements of a gas turbine for which LES is reported: (a) \& (b), swirler; (c) single sector domain taking into account the swirler (orange), the flame tube (dark grey) and the chamber casing (yellow); (d) full annular configuration with all sectors, swirlers and the entire flame tube. The particular example corresponds to a reverse combustor design. (For interpretation of the references to color in this figure legend, the reader is referred to the web version of this article.)

\subsubsection{Transient operating conditions}

LES being intrinsically unsteady, one single fully transient flow (i.e. non-statistically stationary) can be obtained. Although such predictions usually require averaging multiple LES [305], individual predictions can guide our understanding of complex phenomena otherwise inaccessible by conventional or industrial numerical tools.

Two transient reacting flows of great interest to the gas turbine manufacturers are the forced ignition or re-ignition and extinction phases of gas turbine engines. Forced numerical ignition studies for aeronautical applications have recently started [306-312] to complement theoretical and experimental works [305,311,313].

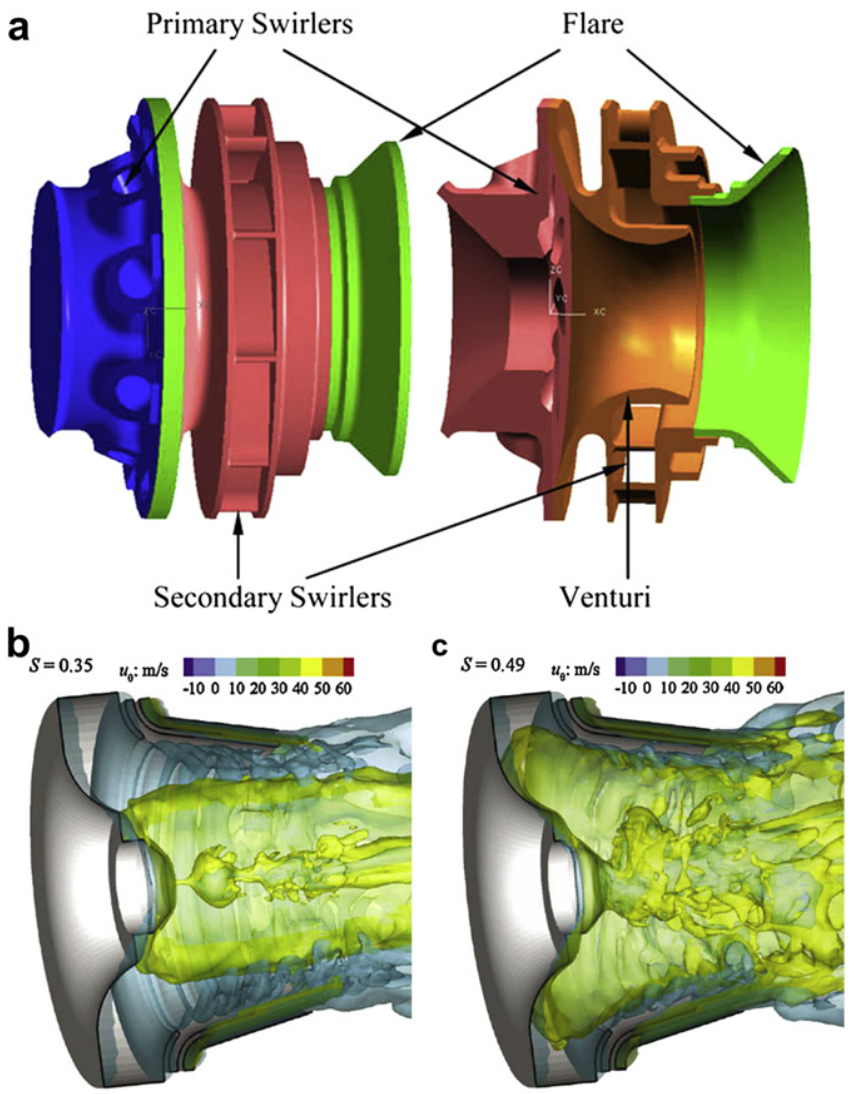

Fig. 22. Real swirler design as reported by [244,250,335]: (a) description of the different elements present on a design for the CFM56 engine and LES predictions of an industrial swirler and whose swirl number is (b) $S=0.35$ and (c) $S=0.49$ (instantaneous views of the azimuthal velocity component vector). 
a

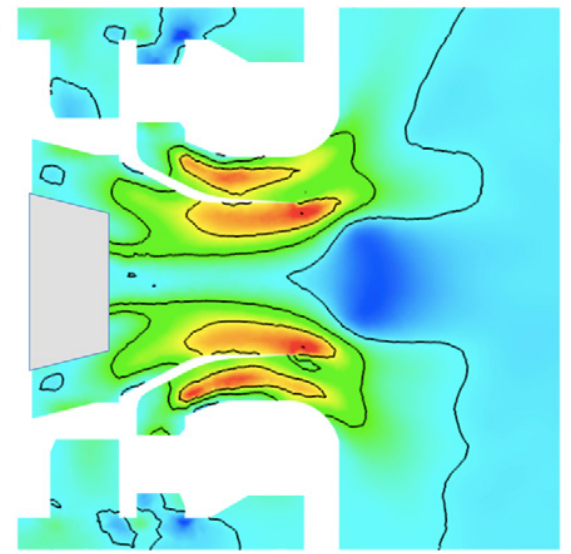

C

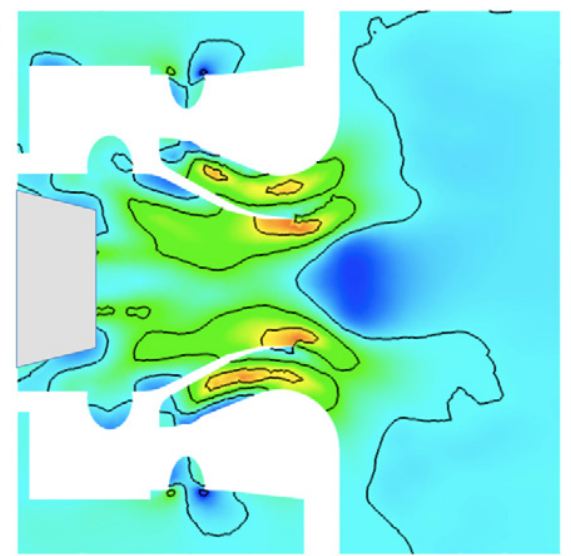

b

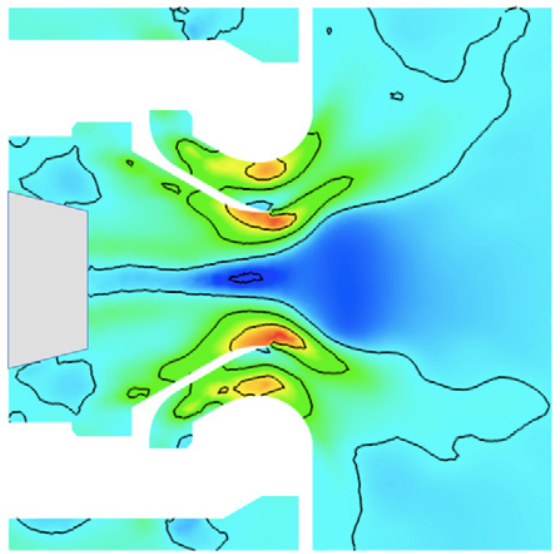

d

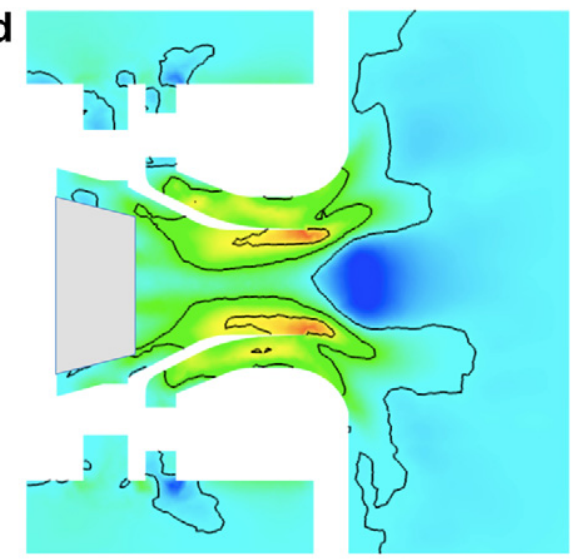

Fig. 23. Real swirler designs evaluated through LES of non-reacting flow: views of the axial mean velocity component, the flow going from left to right (dark blue corresponds to large values of back flow; courtesy of A. Roux from Turbomeca, Safran group). (For interpretation of the references to color in this figure legend, the reader is referred to the web version of this article.)

Laboratory test cases providing a direct comparison between measurements and LES are very promising. An example of forced ignition studied experimentally by [314], Fig. 17, and computed with LES by [310] are presented in Figs. 18 and 19. Two sequences are shown on Figs. 18 and 19 which illustrate the capacity of the approach to address the notion of success or failure the flame stabilization process. For a given energy deposit at a given position but at two different instants of a statistically stationary flow,
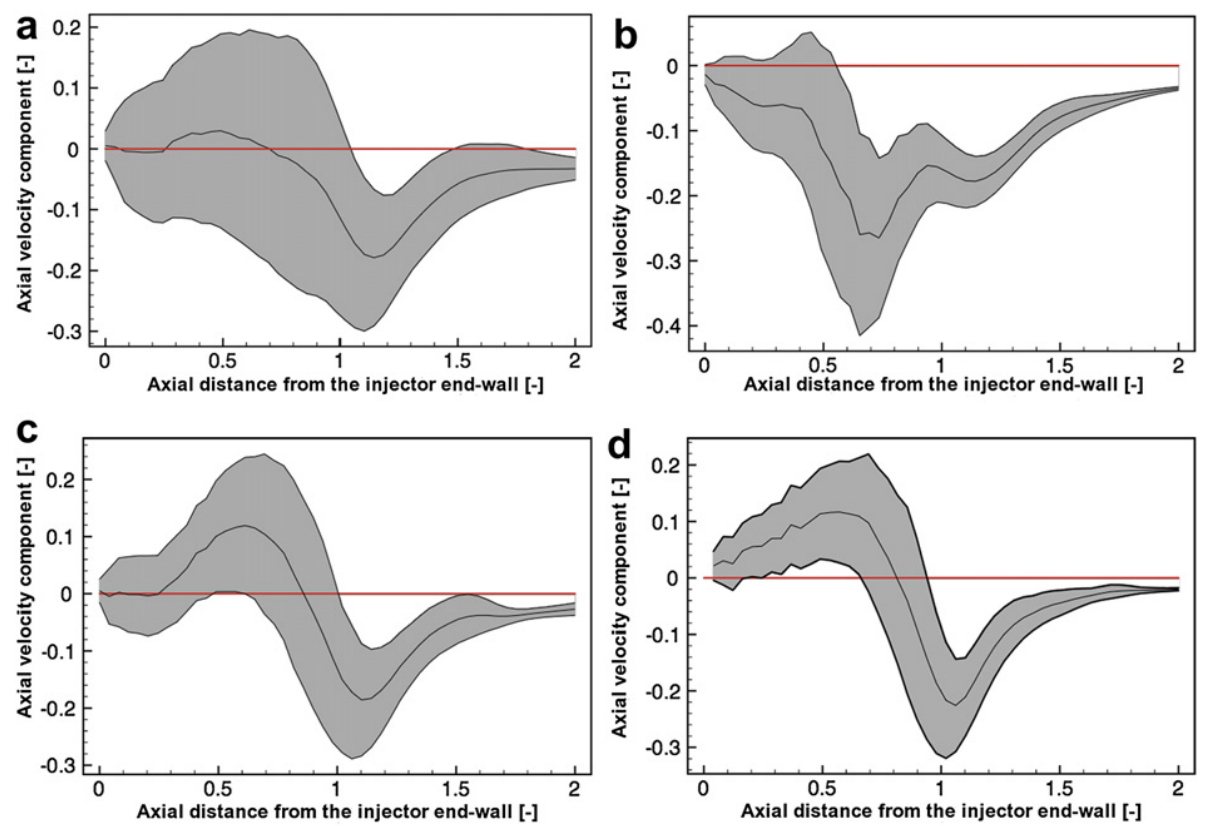

Fig. 24. Mean axial velocity profiles along the symmetry axis of the swirlers of Fig. 23. The superposed grey envelop represents the extent of the potential local and instantaneous value of the axial velocity component obtained by LES (courtesy of A. Roux from Turbomeca, Safran group). 
one observes two distinct transient predictions: a failed one, Fig. 18, and a successful ignition, Fig. 19. Multiple parameters play determining roles in the initially formed flame kernel (if created by a spark or a laser ignitor). Local values of the flammability limits are of course of importance, but so is the turbulence at the flame surface. Naturally, legitimate questions arise from such simulations [308,309,315]. LES remain nonetheless the only tool capable of producing such predictions for high Reynolds number flows.

\section{Real engine combustor simulations}

LES in real engines first appeared in 2004-2008 [48,316-320]. These simulations are more difficult to validate due to the limited set of available measurements and the extreme simplifications introduced in comparison to real operating burners. This section reviews recent LES performed for gaseous reacting flows in real engine combustors. The primary objective of the discussion is to provide a status and highlight the added value of LES for industrial flows.

\subsection{Specific features and missing links}

Performing LES in real chambers imposes geometrical or physical simplifications:
- Boundary conditions (inflow, outflow conditions and impedances): real burners are fed by a compressor and blow into a turbine, Fig. 20(a). These devices control the inflow and outflow conditions of the combustion chamber. Acoustically they induce impedances which need to be evaluated if LES are to be representative of a real operating condition of the engine. Contrarily to most experimental set-ups, fuel and air enter the combustor through multiple inlets and yield a partially premixed environment. The major consequence of these technological choices is that the local flame combustion modes and regimes or the local value of the equivalence ratio are not known.

- Cooling devices: Technological effects (multi-perforated plates, dilution jets, films...), Fig. 20(b), are present in all parts of the combustion chamber to make sure that the combustor walls can durably sustain the hot product temperatures issued by combustion.

- Fuels (liquid and heavily carbonated fuels or even multicomponent fuels): Most real aeronautical engines operate on liquid fuels, Fig. 20(c). Modeling sprays raises a new and significant difficulty (compared to gas combustion). An additional complexity is due to the kinetic models required for such fuels: describing the chemistry of hydrogen in an academic experiment can be done but this exercise remains impossible
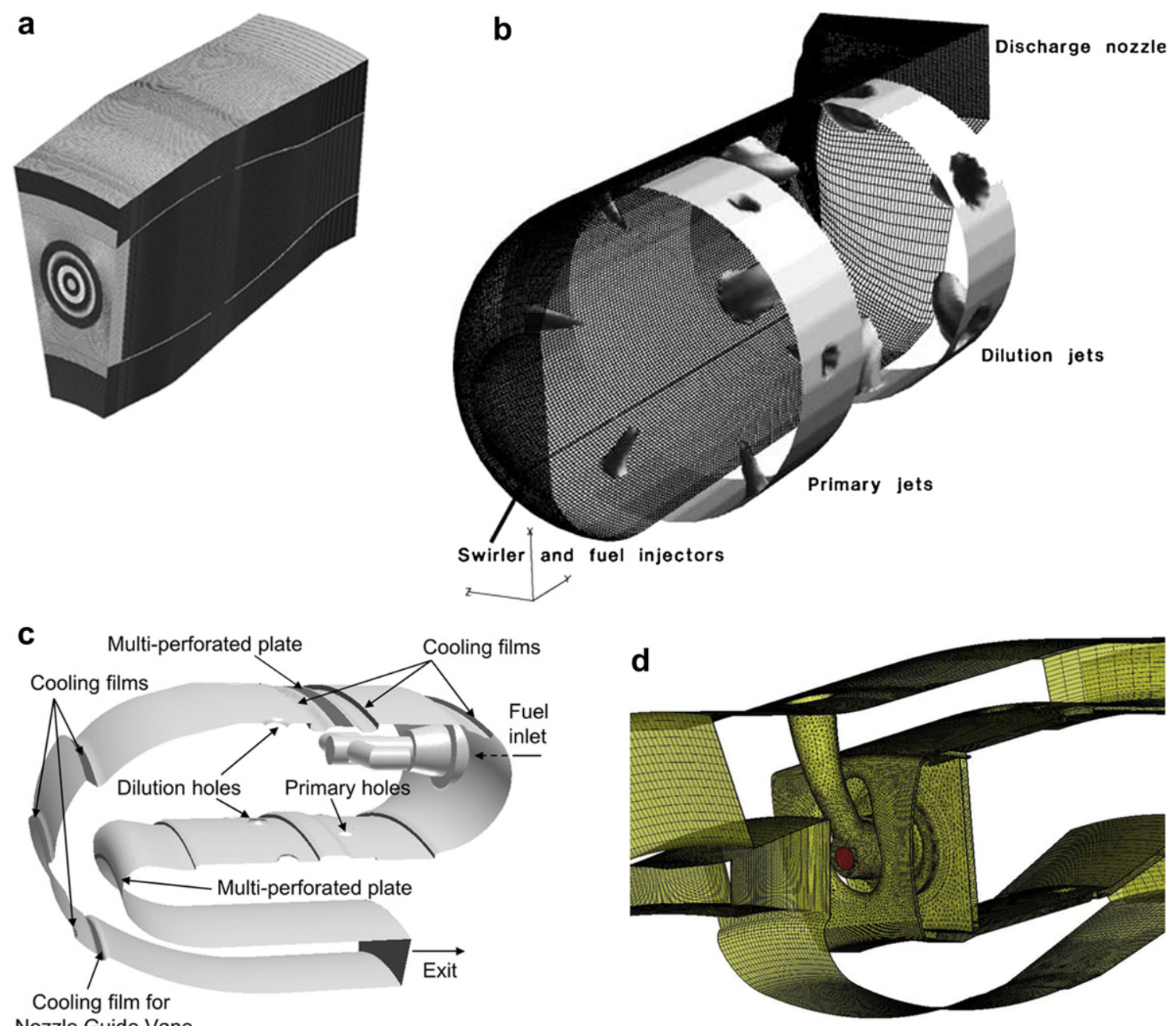

Nozzle Guide Vane

Fig. 25. Single sector LES: views of the different computational domains treated by LES $[48,316-320]$. 
today for the multi-component fuels with long carbon chains found in gas turbines.

On-going researches focus on these difficulties through various mathematical tools and developments that may be integrated in a LES solver targeting real gas turbines. Interested readers are pointed to the homogenization techniques for multi-perforated walls [321-323], Euler/Euler [324-328] and Euler/Lagrange [329,330] formalisms for two-phase flows as well as on-going developments in the field of primary and secondary atomization [331]. In parallel and to improve the prediction of the thermal environment of such devices, recent contributions address multi-physics type of solutions where wall heat transfer [332,333] and/or radiation [334] are coupled to LES.

\subsection{Current state-of-the-art LES for real engine combustors}

Provided that modelers accept necessary simplifications, real engine LES are currently possible as detailed in Table 5 . Since the first published results [48,316-320], multiple laboratories and industrial groups have produced such simulations with different tools and modeling strategies. The results can be divided into three categories related to the geometrical extent of the computation domain and the technological device characterized numerically:

- Non reacting swirler simulations: One of the first steps for combustion chamber engineers is to guaranty that the swirlers, Fig. 21(a) \& (b), will provide the desired flow field and fuel distribution for a proper flame stabilization. Because of the complexity of this device, Figs. 20(c) and 21(a) \& (b), direct flow visualization by cheap diagnostics is not accessible to industry. So swirler design and characterization rely on RANS predictions. These results can be improved or complemented by LES predictions which are more expensive but provide a unique access to the cold flow dynamics before final assembly and testing.

- Single sector simulations: Simulations of the flame tube alone, with the swirler and including or not the chamber casing, are used to predict flame positions, local flame dynamics and exit temperature profiles, Fig. 21(c).

- Full annular burner simulations: The previous simulations can be extended by taking into consideration the entire combustion chamber: i.e. its full complexity in the azimuthal direction, Fig. 21(d). These computations are usually relevant to industry for azimuthal burner thermo-acoustic stabilities, potential long distance flame interactions, fully transient phenomena or geometrical singularities preventing any hypothesis on the azimuthal periodicity of the flow.

\subsubsection{Swirler simulations}

Real swirlers include complex flow passages or veins with multiple obstacles and wing profiles that impose a rotating motion to the air streams. Current technologies involve an increasing number of veins that can be co or counter rotating, axially or radially oriented, Fig. 22(a). In order to guide the flow and control the mass flow rate (i.e. flow split), the shape of the veins is usually highly convoluted and optimized. These Venturi, flares and separators, Fig. 22(a), control the swirl number of each passage. Most importantly, the swirler is the component of the combustor through which fuel feeds the burner. Fuel injection points may be multiple, located at various locations within the swirler and based a

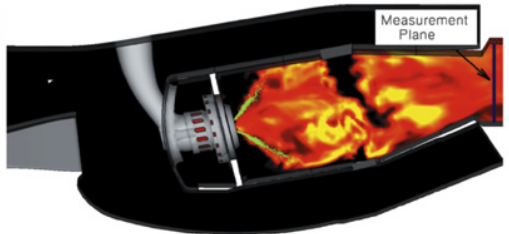

C

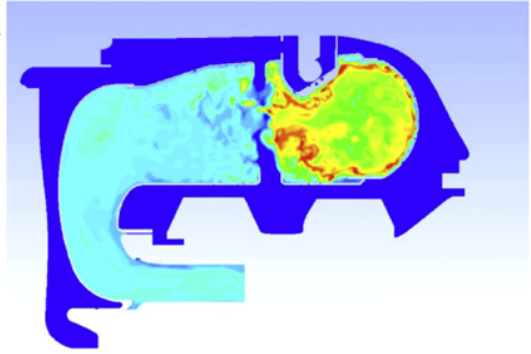

e

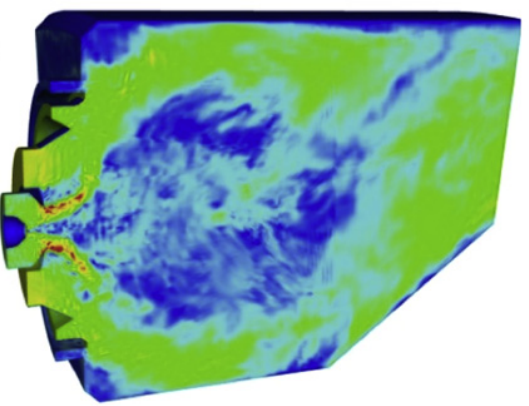

b

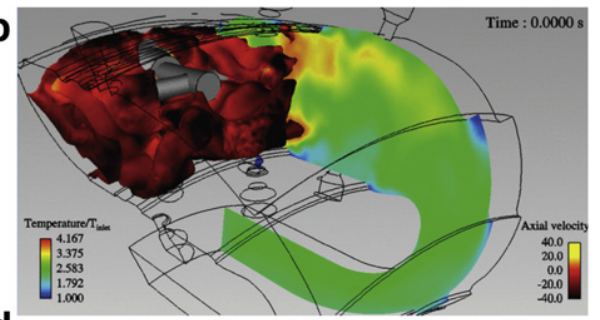

d

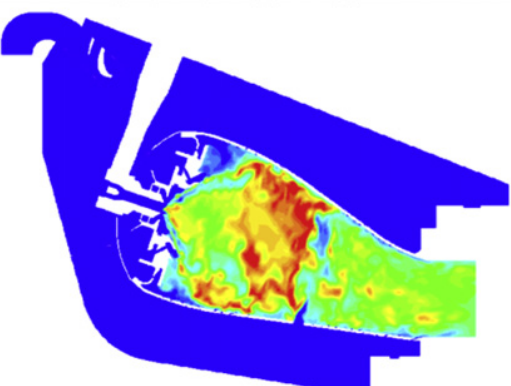

f

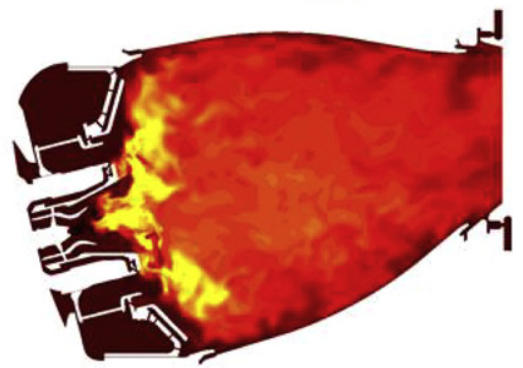

Fig. 26. Typical views of aeronautical gas turbine engine LES: instantaneous field prediction for (a) Pratt \& Withney engine [318], (b) a helicopter engine from Turbomeca (Safran group) [319], (c) and (d) two combustor concepts from Honeywell [365,366], (e) a Rolls-Royce lean burn engine [367] and (f) a GE aviation engine [368]. 
on various types of technologies. In real applications, these injection points can also be operated simultaneously or independently depending on the operating condition of the engine (idle, ignition sequence, cruise or full power). In more advanced designs, fuel staging is also used in control strategies to avoid thermo acoustic instabilities.

With such complex systems, the main difficulty resides in the engineer's ability to guaranty a clear understanding of the mean flow patterns. The main difficulty is to properly predict the IRZ relative position with respect to the chamber end-wall or inner end-wall of the swirler. Two swirled flows (Fig. 22(b) \& (c)) were investigated by LES $[242,243]$ to assess flow dynamics and more specifically the position and breakdown of the IRZ as discussed in details in [244]. A second example is shown on Fig. 23 which presents the mean axial velocity component predicted by LES for four different concepts of swirler. The position of the IRZ in front of the swirler, can change even for small geometrical modifications. For example, Fig. 23(b) shows that in some cases, the IRZ penetrates all the way inside the component producing different flow opening angles at the exit of the device. The added value of LES is also to give reliable access to the unsteady features of the flow as illustrated on Fig. 24: the mean axial velocity component profile along the axis of the four designs with the envelope of resolved fluctuating component (i.e.: $\left.+/-u^{\prime}\right)$. Of course, qualifying one design compared to another is a matter of choice and depends on the objective of the engineer.

A typical example related to thermo-acoustic instabilities, is the analysis and assessment of the swirler flow reactivity to external forcing. For such analyses [335], the flow response to various fluctuations is possible by LES. In [335], the response of a radial swirl injector to mass flow rate perturbations is obtained for a wide range of frequencies. Similarly to [302] but in nonreacting conditions, the flow response to acoustic forcing provides understanding of the leading mechanisms governing the acoustic admittance of the devices. These computations help to qualify the impact such oscillations can have on the mixing of air and fuel $[250,277,302]$ prior to combustion in the main chamber.

\subsubsection{Single sector simulations}

Pioneering studies on real industrial combustion chamber $[48,316,318,319]$ mainly focused on a single sector description of the full annular gas turbine burner (Figs. 25 and 26) thereby imposing an axi-periodic hypothesis on the flow realization. This simplification is mainly justified by the need to reduce the computational overhead of LES. The primary objective of such computations, Fig. 26, is to qualify LES strategies and codes on industrial problems and assess the gain offered by the method when compared to RANS tools.

Since experimental measurements of the reacting flow in real configurations are scarce, industrial design criteria are used to assess any new numerical approach. Very limited information is known on real engines and design parameters are only indirect diagnostics: typically, while academic experiments provide velocity, temperature and species fields in the whole combustor, real combustor data correspond only to a few temperature measurements at the chamber outlet and one value of the total flow rate. One important quality parameter scrutinized by engineers is the mean exit temperature field of the combustion chamber because it controls the lifetime of the turbine blades. Improved
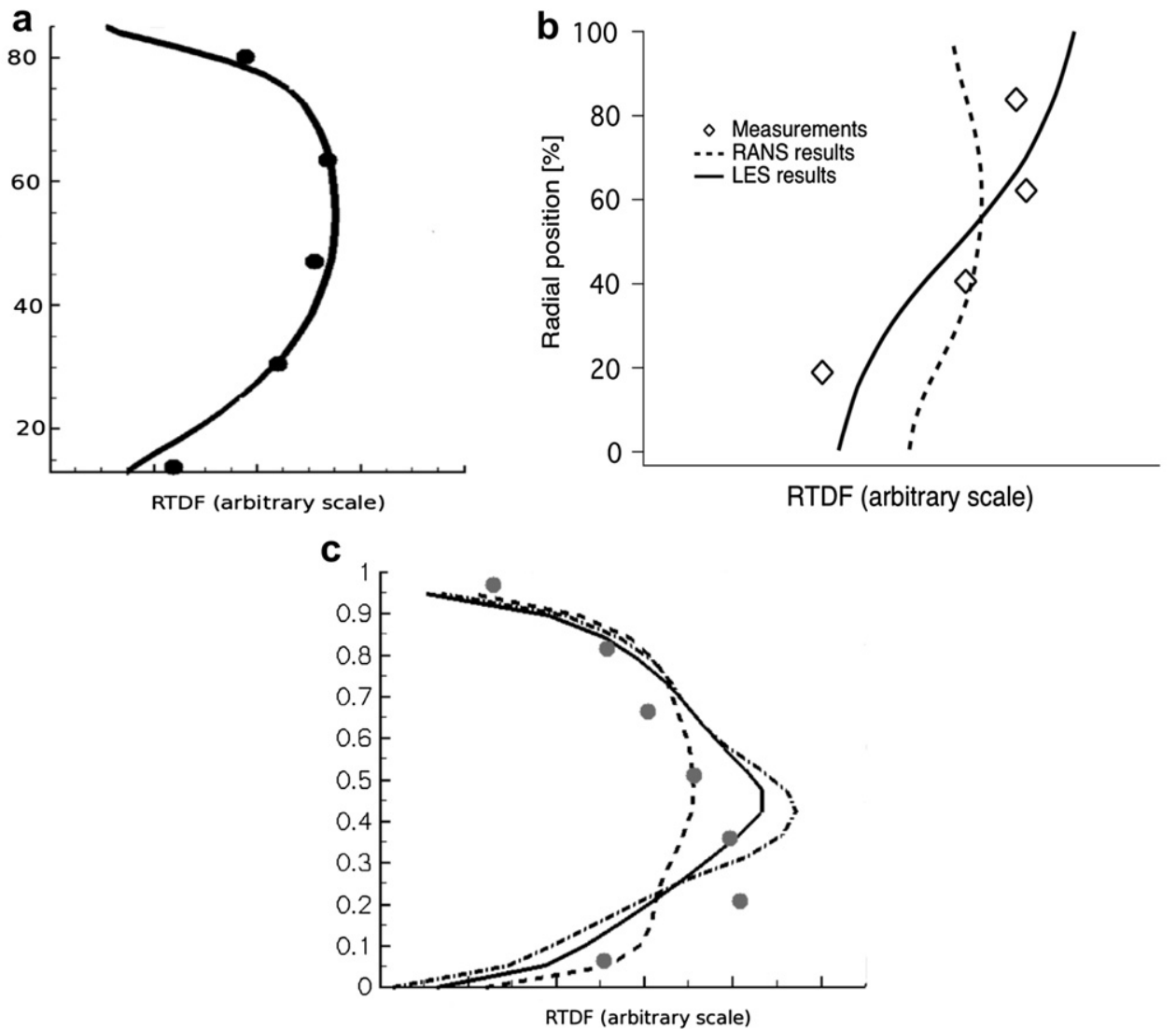

Fig. 27. Outlet normalized temperature profiles (RDTF) obtained numerically by LES, RANS and experiments for different real combustors: (a) Pratt \& Whitney engine (LES, line and experiment, symbols) in the plane identified in Fig. 26 [318] (a); (b) Turbomeca engine (LES, RANS and experiment) [319] and (c) Rolls-Royce engine (LES with and without the casing and RANS with the casing) [48]: Symbol, rig measurements; -, LES of liner plus annuli; - -, LES of liner only; and -.-, RANS simulation of liner only 
estimates of this field induce better known limits of the engine operation and effectively translate in turbine blades that are more effectively designed and cooled. Ideally the exit combustor temperature profile should be well homogenized thanks to an efficient mixing in time and space of the hot products of combustion with cooler air coming from dilution jets and wall films [336]. Of course this optimal point is difficult to reach in combustors which are more and more compact and require larger amount of air to go through the swirler (to improve premixing needed for pollutant reduction). Long and difficult design iterative loops based on RANS are thus needed to locate the primary and secondary jet positions that meet specific objectives.

Preliminary LES of single sector computations with similar geometrical constraints as the one encountered with RANS were
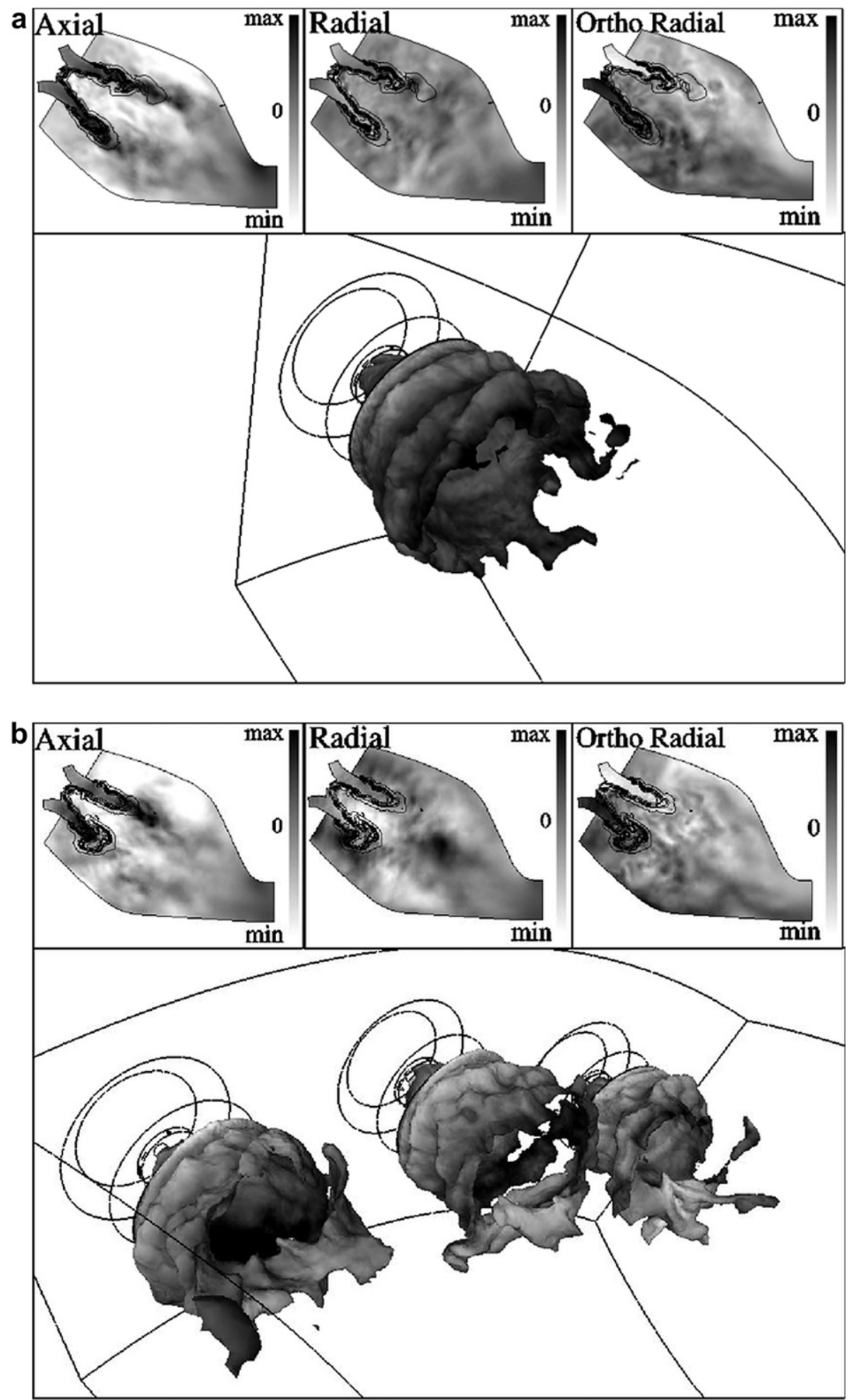

Fig. 28. FTF predictions of an industrial burner by treating (a) one single and (b) three burner forced LES [349]. 
rapidly produced $[48,316,318,319]$. Comparisons are obtained for the Radial Temperature Distribution Function (RTDF), which corresponds to the radial representation of the mean azimuthal variations of the temperature elevation relative to a reference value [319]. Fig. 27 shows that LES provide better representations of this crucial parameter for different types of engines. These preliminary studies, also point out that to capture the mixing process between hot and fresh gases, the flame stabilization mechanism, the swirler [316] as well as part of the casing [48] may need to be included in the computational domain [337]. To reduce the boundary condition impact, an ad-hoc strategy is to extend the computational domain to zones where the boundary conditions are known [338-341].

Local mesh resolution effects have also been investigated $[320,342]$. Preliminary conclusions are in agreement with theoretical analyses and observations obtained on laboratory-scale burners. However, in complex configurations, mean fields seem relatively insensitive to mesh resolution when looking at velocity statistics. Conclusions are not as clear for reacting LES especially for combustion quantities. Turbulent combustion modeling effects have been obtained [343] for a fixed grid resolution and show reasonable agreement between all mean fields irrespectively of the model used (CMC-LES [344] or EBU-LES [343]). All these findings confirm the robustness of LES for an industrial use.

Similarly to the predictions obtained in the research context of thermo-acoustic instabilities, single sector real engine LES provide estimates of FTF's that are otherwise not accessible or very costly to produce experimentally $[345,346]$. These FTF's can then be used along with thermo-acoustic solvers (Helmholtz or network models) to determine the thermo-acoustic stability map of a burner [298,299,347,348]. For such LES [349], the compressible solver needs to consider an acoustically open numerical setup (no acoustic reflection at the inflow and outflow conditions of the LES computational domain). Acoustic forcing of the inflow (or outflow) [290-292,350] is then applied to determine the frequency dependent function that is the FTF [351-353], Fig. 28. It is important to underline at this point that current FTF estimates obtained with such single sector LES impose specific constraints. Typically, such FTF's are representative of a flame response issued by acoustic modes inducing flow perturbations going through the studied burner. In some situations, azimuthal acoustic modes, often triggered in annular combustors, may arise from flame interactions issued by purely azimuthal modes that can be potentially transparent from the swirler passage point of view. These issues and potential limits are clearly unanswered today and are being investigated by different institutions in the world.

Finally, fully transient phases as encountered in the ignition of a burner are also available in real burners [312,344,354]. These predictions are more difficult to assess.

\subsubsection{Full annular burner simulations}

The increase in computing power combined with the potential simplification of the boundary conditions has lead to computations of complete chambers. Typically, full annular combustor demonstrations have been produced recently [342,355-358]. Such extended computational domains are justified only if information proceeds in the azimuthal direction and can not be properly captured with a single sector hypothesis: for example to simulate flame propagation from a burner to the next after ignition of a flame kernel [307], neighboring flames that interact with each other or the existence of an azimuthal thermo-acoustic instabilities [356].

Fig. 29 presents snapshots of two full burner LES obtained by considering the entire geometry of the burner located between the
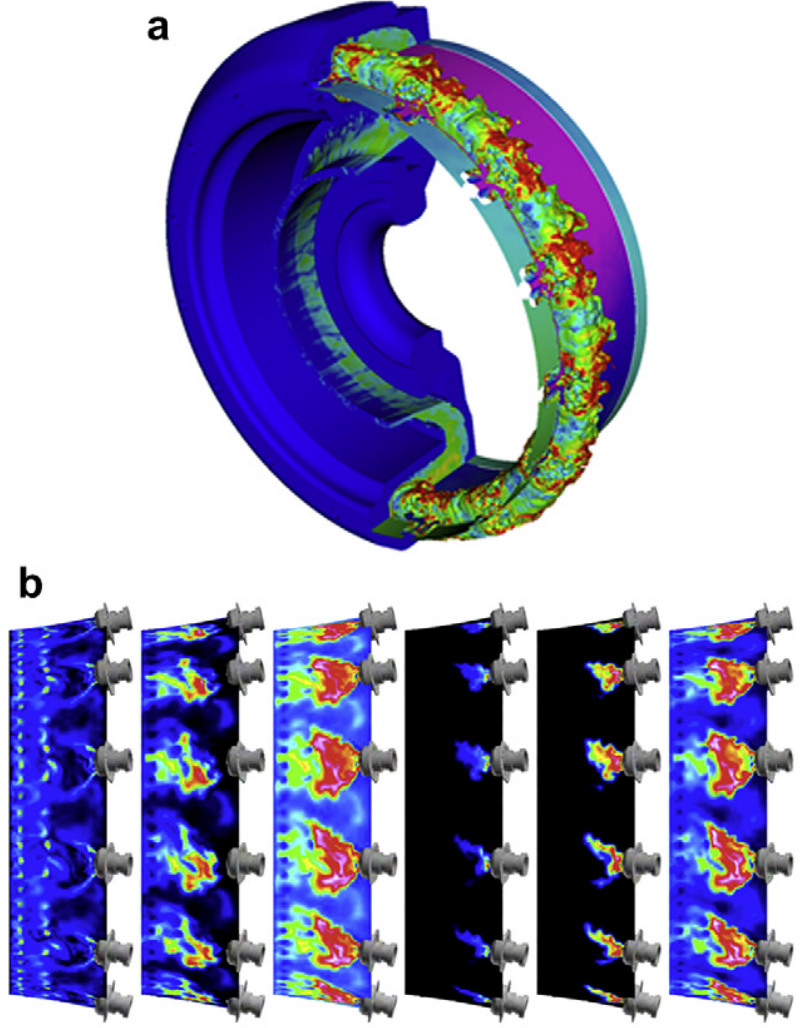

Fig. 29. Instantaneous snapshot of LES predictions in full annular real combustors: (a) from [358] and (b) from [342,355-357].

compressor and the turbine for (a) a Turbomeca burner [342] and (b) the CESAR combustor [358]. In both predictions pressure oscillations are reported and linked to azimuthal thermo-acoustic modes. Compared to single sector LES [355,357,358], the aerodynamics within the combustion chamber differ and the secondary jet mixing with the hot products changes from burner to burner. Reports on the LES behaviors and indirect observations on the real engines confirm the good quality of the predictions thereby providing some confidence in the ability of LES to at least reproduce macroscopic unsteady flow in real engines. These results not only provide a demonstration of the current status of advanced LES solvers when used on massively parallel computers but also give access to new sets of data. Indeed such unsteady fields need now to be studied to feed the design chain and complement design assessments based on RANS. From a theoretical point of view, these simulations greatly contribute to our understanding of azimuthal thermo-acoustic instabilities in complex burners and open new perspectives in terms of investigations. Indeed and despite the fact that great care still needs to be taken, resulting limit-cycles and their triggering mechanisms are clearly of great importance.

Finally, it is interesting to discuss the first simulation of ignition in a full annular chamber [307]. For this demonstration, the problem of burner to burner flame propagation is specifically addressed: two opposite torch ignitors located in an annular combustion chamber provide the initial energy to the non reacting flow mixture of air and fuel, Fig. 30(a). As time proceeds the resulting flames propagate in opposite directions to ignite the different neighboring sectors of the entire combustor, Fig. 30(b)-(c). Such a sequence is inherently unsteady and goes from a statistically stationary cold flow engine to a fully statistically stationary reacting flow corresponding to an engine ready for operation. LES [307] offer a first view of that phase that could be envisioned by engineers only indirectly. Indeed, test- 

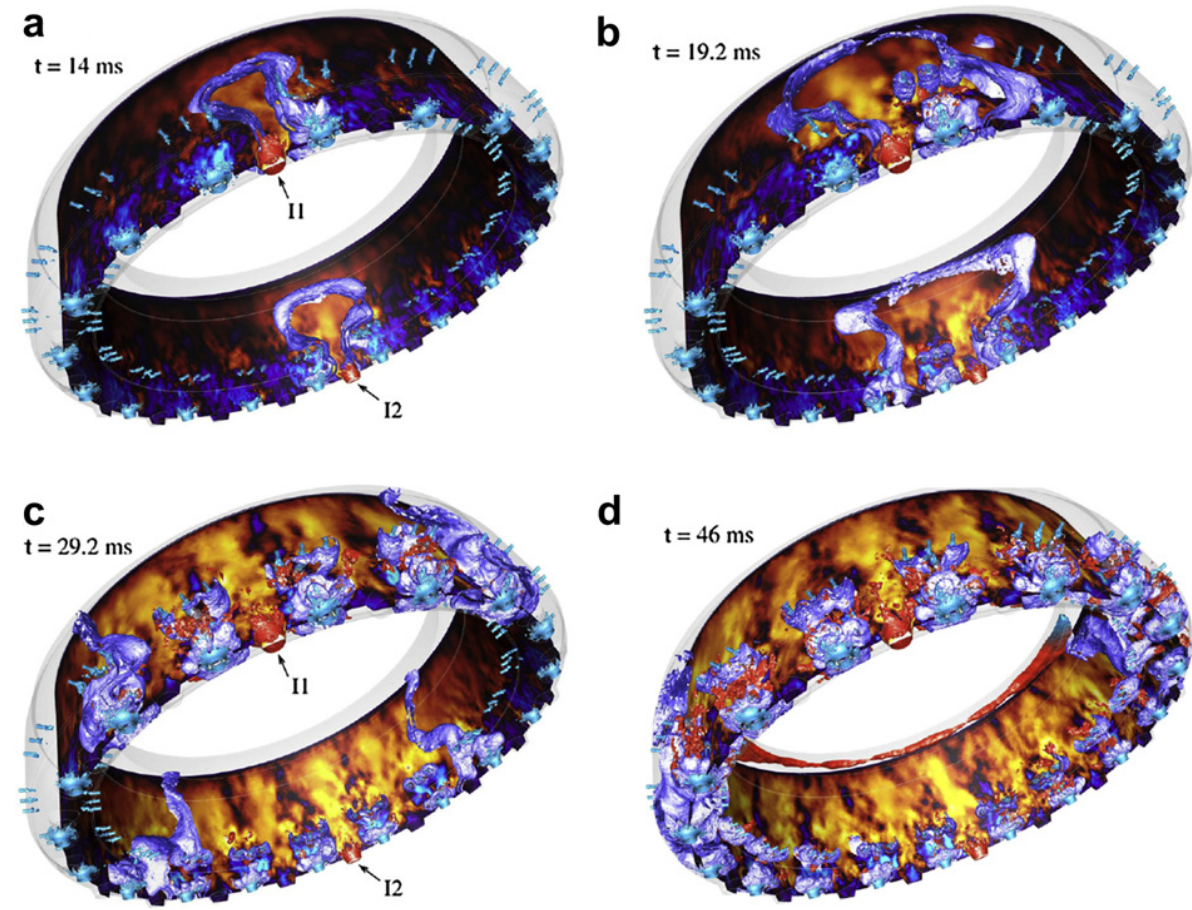

Fig. 30. LES of a light-around sequence obtained for an annular combustion chamber [307].

bench facilities can in this case at best provide an integrated view of the process or indirect diagnostics such as the engine pressure and power output while the light-around proceeds. Results reveal that the whole ignition process takes around $40 \mathrm{~ms}$, that the flame front is propagating azimuthally at $20 \mathrm{~m} / \mathrm{s}$ and is mainly driven by gas expansion.

\section{Conclusions and perspectives}

Over the last decade LES of turbulent reacting flows has received great interest from the scientific community. This interest now spreads to companies because LES are the only alternative to the two extreme numerical tools available to the combustion community: Direct Numerical Simulations (DNS) and Reynolds Average Navier-Stokes Simulations (RANS) [359].

- In the former, the turbulent reacting flow is addressed in a brute force way and all scales are resolved in space and time by the numerical scheme which needs to be highly accurate and used in very small well-controlled computational domains to ensure reliable and stable simulations.

- In the latter, the governing equations are first mathematically recast into a new set of equations (usually time-independent) governing the spatial evolution of the mean flow quantities. All scales of interactions require modeling which is a quite difficult task.

Despite its intrinsic difficulties, RANS has rapidly entered the design chain of current industrial gas turbine manufacturers. It has benefited from intense researches as well as modeling contributions and is today very cost effective. DNS is almost model free but is not applicable to industrial applications because it is too computer intensive and unable to deal with Reynolds numbers or the geometrical complexities of real applications. The computing power for such exercises is simply not accessible. It is nonetheless a very valuable scientific tool that benefits from the current and future evolutions of super-computing and has become a key element of the recent modeling strategies.

LES represent partially the unsteady dynamics of the flows present in DNS and required for improved predictability while alleviating the modeling effort needed with RANS. Being a fully unsteady formalism, LES computer cost is typically 100 times larger than RANS. However, since only the large-scale flow quantities are solved for, the Reynolds number and Damkhöler number flows accessible to LES are greatly extended compared to DNS. As a result, if one takes into account the increasing computing power, LES can be performed today for many burners, in one night (like RANS a few years ago) with a precision which is not very far from DNS.

Despite such progresses, comprehensive and detailed studies are still needed to further extend LES to real combustors. Aside from the recurring difficulty of properly addressing turbulent reacting flows, which is independent of the numerical approach, grid resolution plays a determining role in LES model assessments. This specific issue becomes more complicated in industrial applications where combustion modes and regimes are a priori unknown and grid resolution is far from being uniform. The influence of boundary conditions on the predictions also appears to be of importance for LES of laboratory or real engine configurations. Recent developments in algorithmic open new perspectives on the effective contributions of modeling compared to numerics. The new generations of solvers allow to include geometrical complexities appearing in real applications thereby providing more realistic and predictive simulations. They also lead to more systematic analyses of the modeling hypotheses needed to produce LES closures with reduced numerical impact. At the same time and because of the reduced size of the local mesh resolution accessible to these codes, new LES closures can arise focusing on new formalisms oriented toward mixing and flame inner structures subject to quasi-fully resolved turbulent fields.

The last decade has seen many new applications of LES to real combustors. Transient reacting flows such as forced ignition sequences of burners are now numerically addressed in parallel to 
advanced experiments with temporally resolved diagnostics. Thermo-acoustic instability is also a field which now relies as much on numerics as on experiments. LES of both problems have appeared to assess real engine combustion chambers and preliminary results are very encouraging. More simulations are being produced within industry to complement RANS predictions and improve the design cycle of the next generation of aeronautical engines. However, this ultimate objective still requires further modeling to be able to take into account the multi-phase flow nature of the fuel alimentation of real engines. Better estimates of the thermal environment will probably require considering conduction, radiation and technological solutions that are still difficult to address directly numerically with the available grid resolution or even theoretically in the context of LES.

\section{Acknowledgments}

The authors thank the CFD Team at CERFACS: trainees, PhD's, Post-Doctoral fellows, staff as well as industrial partners who contributed in producing some of the results and points discussed here. Research partners from European projects, Universities and Industries are acknowledged for sharing their results for this review and for participating in fruitful discussions and exchanges which make the turbulent combustion field of research a very stimulating environment for students, young researchers and engineers.

\section{References}

[1] Von Karman TLH. On the statistical theory of isotropic turbulence. In: Proceedings of the Royal Society of London, 1938.

[2] Hinze JO. Turbulence. McGraw-Hill; 1959.

[3] Tennekes H, Lumley JL. A first course in turbulence. Cambridge: M.I.T. Press; 1972.

[4] Launder BE, Spalding DB. The numerical computation of turbulent flows. Comput Meth Appl Mech Eng 1974;3(2):269-89.

[5] Reynolds WC. Computation of turbulent flows. Ann Rev Fluid Mech 1976;8: 183-208.

[6] Lumley JL. Computational modeling of turbulent flows. Adv Appl Mech 1978; 18:123-76.

[7] Lesieur M. Turbulence in fluids. Fluid mechanics and its applications. Kluwer Academic Publishers; 1990

[8] Chassaing P. Mécanique des fluides, Éléments d'un premier parcours. Cépaduès-Éditions; 2000.

[9] Chassaing P. Turbulence en mécanique des fluides, Analyse du phénomène dans une perspective de modélisation à l'usage de l'ingénieur. Institut National Polytechnique de Toulouse; 2000.

[10] Pope SB. Turbulent flows. Cambridge University Press; 2000.

[11] Zukoski EE, Marble FE. Combustion researches and reviews. Paris: Butterworths Scientific Publications; 1955. p. 167.

[12] Spalding DB. Mathematical models of turbulent flames, a review. Combust Sci Tech 1976;13:3-25.

[13] Jones WP, Whitelaw JH. Calculation methods for reacting turbulent flows: a review. Combust Flame 1982;48:1-26.

[14] Kim J, Moin P, Moser R. Turbulence statistics in fully developed channel flow at low Reynolds number. J Fluid Mech 1987;177:133-66. http://dx.doi.org/ 10.1017/S0022112087000892.

[15] Spalart PR. Direct simulation of a turbulent boundary layer up to $R e_{\theta}=1410$. J Fluid Mech 1988;187:61-98. http://dx.doi.org/10.1017/S0022112088000345.

[16] Jiménez J, Moin P. The minimal flow unit in near-wall turbulence. J Fluid Mech 1991;225:213-40. http://dx.doi.org/10.1017/S0022112091002033.

[17] Sumitani Y, Kasagi N. Direct numerical simulation of turbulent transport with uniform wall injection and suction. Am Inst Aeronaut Astronaut J 1995; 33(7):1220-8

[18] Kawamura H, Ohsaka K, Abe H, Yamamoto K. DNS of turbulent heat transfer in channel flow with low to medium-high Prandtl number fluid. Int J Heat Fluid Flow 1998;19:482-91. http://dx.doi.org/10.1016/S0142-727X(98) 10026-7.

[19] Moser R, Kim J, Mansour N. Direct numerical simulation of turbulent channel flow up to $\operatorname{Re}_{\tau}=590$. Phys Fluids 1999;11(4):943-5. http://dx.doi.org/10. 1063/1.869966.

[20] Leonard A. Energy cascade in large eddy simulations of turbulent fluid flows. Adv Geophys 1974;18(A):237-48.

[21] Ferziger J. Large eddy simulations of turbulent flows. Am Inst Aeronaut Astronaut J 1977;15(9):1261-7.
[22] Rogallo R, Moin P. Numerical simulation of turbulent flows. Ann Rev Fluid Mech 1985;17:99-137.

[23] Mason PJ. Large-eddy simulation: a critical review of the technique. QJ Royal Meteorol Soc 1994;120(515):1-26 (A)

[24] Piomelli U, Chasnov JR. Large eddy simulations: theory and applications. In: Hallback $\mathrm{H}$, Henningson $\mathrm{D}$, Johansson A, Alfredsson P, editors. Turbulence and transition modelling. Kluwer Academic Publishers; 1996. p. 269-336.

[25] Lesieur M, Metais O. New trends in large-eddy simulations of turbulence. Ann Rev Fluid Mech 1996;28:45-82.

[26] Ferziger J. Large eddy simulation: an introduction and perspective. In: Métais O, Ferziger J, editors. New tools in turbulence modelling. Les Editions de Physique - Springer Verlag; 1997. p. 29-47.

[27] Lesieur M. Turbulence in fluids. In: Fluid Mechanics and its applications. 3rd rev., vol. 40. Kluwer Academic Publishers; 1997.

[28] Piomelli U. Large-eddy simulation: achievements and challenges. Prog Aerospace Sci 1999;35:335-62.

[29] Meneveau C, Katz J. Scale-invariance and turbulence models for large eddy simulation. Ann Rev Fluid Mech 2000;32:1-32.

[30] Sagaut P. Large eddy simulation for incompressible flows. Springer; 2002.

[31] Poinsot T, Veynante D. Theoretical and numerical combustion. 2nd ed. R.T. Edwards; 2005.

[32] Poinsot T, Kestens T, Nicoud F. Using computational fluid dynamics to study flow control. Comput Meth Cont Appl 2002;16:327-46.

[33] Kolmogorov AN. The local structure of turbulence in incompressible viscous fluid for very large Reynolds numbers. C R Acad Sci USSR 1941;30:301.

[34] Kolmogorov AN. A refinement of hypothesis concerning the local structure of turbulence in a viscous incompressible fluid at high Reynolds number. J Fluid Mech 1962;62:82.

[35] Linan A. The asymptotic structure of counterflow diffusion flames for large activation energies. Acta Astronautica 1974;1:1007.

[36] Cuenot B, Poinsot T. Asymptotic and numerical study of diffusion flames with variable Lewis number and finite rate chemistry. Combust Flame 1996;104: 111-37.

[37] Vervisch L, Poinsot T. Direct numerical simulation of non premixed turbulent flames. Ann Rev Fluid Mech 1998;30:655-92.

[38] Givi P. Model-free simulations of turbulent reactive flows. Prog Energy Comb Sci 1989;15:1-107.

[39] Givi P. Spectral and random vortex methods in turbulent reacting flows. In: Williams F,P,L, editor. Turbulent reacting flows. Academic Press; 1994. p. 475-572.

[40] Poinsot T, Haworth D. DNS and modelling of the interaction between turbulent premixed flames and walls. In: Proc. of the summer program. Center for Turbulence Research, NASA Ames/Stanford Univ.; 1992. p. 307-24.

[41] Poinsot T, Candel S, Trouvé A. Application of direct numerical simulation to premixed turbulent combustion. Prog Energy Comb Sci 1996;21:531-76.

[42] Bell J, Day M, Grcar J, Lijewski M, Driscoll JF, Filatyev S. Numerical simulation of a laboratory-scale turbulent slot flame. Proc Combust Inst 2007;31: 1299-307.

[43] Bell J, Cheng R, Day M, Beckner V, Leijewski M. Interaction of turbulence and chemistry in a low-swirl burner. J Phys: Conf Series 2008;125:012027.

[44] Gao X, Groth C. A parallel solution-adaptive method for three-dimensional turbulent non-premixed combusting flows. J Comput Phys 2010;229: 3250-75.

[45] Aspden A, Day M, Bell J. Characterization of low Lewis number flames. Proc Combust Inst 2011;33(1):1463-71.

[46] Aspden A, Day M, Bell J. Lewis number effects in distributed flames. Proc Combust Inst 2011;33(1):1473-80.

[47] Day M, Bell J, Bremer P, Pascucci V, Beckner V, Lijewski M. Turbulence effects on cellular burning structures in lean premixed hydrogen flames. Combust Flame 2009;156(5):1035-45.

[48] James S, Zhu J, Anand M. Large eddy simulation as a design tool for gas turbine combustion systems. Am Inst Aeronaut Astronaut J 2006:44:674-86.

[49] Aldama AA. Lecture notes in engineering, vol. 49. New York: Springer-Verlag; 1990.

[50] Moureau V. Private communication, 2006.

[51] Fureby C, Tabor G. Mathematical and physical constraints on large-eddy simulations. Theory Comput Fluid Dyn 1997;9:85-102.

[52] Ghosal S. An analysis of numerical errors in large eddy simulations of turbulence. J Comput Phys 1996;125:187-206.

[53] Vasilyev OV, Lund TS, Moin P. A general class of commutative filters for les in complex geometries. J Comput Phys 1998;146:82-104.

[54] Morinishi Y, Vasilyev OV. A recommended modification to the dynamic twoparameter mixed subgrid scale model for large eddy simulation of wall bounded turbulent flow. Phys Fluids 2001;13(11):3400-10. http://dx.doi. $\operatorname{org} / 10.1063 / 1.1404396$

[55] Moureau VR, Vasilyev OV, Angelberger C, Poinsot TJ. Commutation errors in large eddy simulations on moving grids: application to piston engine flows. In: Proc. of the summer program. USA: Center for Turbulence Research, NASA AMES/Stanford University; 2004. p. 157-68.

[56] Speziale CG. Galilean invariance of subgrid-scale stress models in the largeeddy simulation of turbulence. J Fluid Mech 1985;156:55-62.

[57] Vreman B, Guerts B, Kuerten H. Realizability conditions for the turbulent stress tensor in large eddy simulation. J Fluid Mech 1994a;278:351-64. 
[58] Ghosal S. Mathematical and physical constraints on large-eddy simulation of turbulence. Am Inst Aeronaut Astronaut J 1999;37(4):425.

[59] Kravchenko AG, Moin P. On the effect of numerical errors in large eddy simulations of turbulent flows. J Comput Phys 1997;131(2):310-22. http:// dx.doi.org/10.1006/jcph.1996.5597.

[60] Muchinsky A. A similarity theory of locally homogeneous anisotropic turbulence generated by a Smagorinsky-type LES. J Fluid Mech 1996;325: 239-60.

[61] Fureby C, Grinstein FF. Monotonically integrated large-eddy simulation of free shear flows. Am Inst Aeronaut Astronaut J 1999;37(5):544-56.

[62] Garnier E, Mossi M, Sagaut P, Deville M. On the use of shock-capturing schemes for large eddy simulation. J Comput Phys 1999;153:273-311.

[63] Grinstein F, Margonin L, Rider W. Implicit large eddy simulation: computing turbulent fluid dynamics. Cambridge University Press; 2007.

[64] Germano M. Turbulence: the filtering approach. J Fluid Mech 1992;238 325-36.

[65] Nallasamy N. Turbulence models and their applications to the prediction of internal flows, a review. Comput Fluids 1987;15(2):151-94.

[66] Speziale CG. Turbulence modeling for time-dependent RANS and VLES a review. Am Inst Aeronaut Astronaut J 1998;36(2):173-84. http://dx.doi. org/10.2514/2.7499.

[67] Pitsch H, Steiner H. Scalar mixing and dissipation rate in large-eddy simulations of non-premixed turbulent combustion. Proc Combust Inst 2000;28: 41-9.

[68] Williams FA. Combustion theory. Menlo Park, CA: Benjamin Cummings 1985.

[69] Peters N. Length scales in laminar and turbulent flames. Numerical approaches to combustion modeling; 1989.

[70] Kuo KK. Principles of combustion. 2nd ed. Hoboken, New Jersey: John Wiley \& Sons, Inc; 2005

[71] Williams FA. A review of some theoretical combustions of turbulent flame structure. In: AGARD conference proceedings, vol. 164; 1975. p. II.1.1-25.

[72] Libby PA, Williams FA. Turbulent combustion: fundamental aspects and a review. In: Williams F, Libby P, editors. Turbulent reacting flows. Academic Press London; 1994. p. 2-61.

[73] Candel S, Veynante D, Lacas F, Darabiha N, Baum M, Poinsot T. A review of turbulent combustion modeling. ERCOFTAC Bulletin 1994;20:9-16.

[74] Vervisch L, Veynante D. Interlinks between approaches for modeling turbulent flames. Proc Combust Inst 2000;28:175-83.

[75] Peters N. Turbulent combustion. Cambridge University Press; 2001.

[76] Veynante D, Vervisch L. Turbulent combustion modeling. Prog Energy Combust Sci 2002;28:193-266.

[77] Riley J. Review of large-eddy simulation of non-premixed turbulen combustion. J Fluids Eng 2006;128(2):209-15.

[78] Pitsch H. Large eddy simulation of turbulent combustion. Ann Rev Fluid Mech 2006;38:453-82.

[79] Favre A. Statistical equations of turbulent gases. In: Problems of hydrodynamics and continuum mechanics. Philadelphia: SIAM; 1969. p. 231-66.

[80] Moin P, Squires KD, Cabot W, Lee S. A dynamic subgrid-scale model for compressible turbulence and scalar transport. Phys Fluids 1991;A 3(11): 2746-57. http://dx.doi.org/10.1063/1.858164.

[81] Erlebacher G, Hussaini MY, Speziale CG, Zang TA. Toward the large-eddy simulation of compressible turbulent flows. J Fluid Mech 1992;238:155-85.

[82] Vreman B, Geurts B, Kuerten H. Subgrid modeling in LES of compressible flow. Appl Sci Res 1995;54:191-203.

[83] Smagorinsky J. General circulation experiments with the primitive equations: 1 . The basic experiment. Mon Weather Rev 1963;91:99-164.

[84] Lilly DK. The representation of small-scale turbulence in numerical simulation experiments. In: Proceedings of the IBM scientific computing symposium on environmental sciences; 1967 . Yorktown Heights, USA.

[85] Scotti A, Meneveau C, Lilly DK. Generalized smagorinsky model for anisotropic grids. Phys Fluids A 1993;5(9):2306-8.

[86] Scotti A, Meneveau C, Fatica M. Generalized smagorinsky model for anisotropic grids. Phys Fluids 1997;9(6):1856-8.

[87] Germano M, Piomelli U, Moin P, Cabot W. A dynamic subgrid-scale eddy viscosity model. Phys Fluids 1991;3(7):1760-5.

[88] Lilly DK. A proposed modification of the germano sub-grid closure method. Phys Fluids 1992;4(3):633-5 [URL LES].

[89] Ghosal S, Moin P. The basic equations for the large eddy simulation of turbulent flows in complex geometry. J Comput Phys 1995;118:24-37.

[90] Deardorff J. The use of subgrid transport equations in a three-dimensional model of atmospheric turbulence. ASME J Fluids Eng 1973;95:429-38.

[91] Schumann U. Subgrid scale model for finite difference simulations of turbulent flows in plane channels and annuli. J Comput Phys 1975;18: 376-404.

[92] Meneveau C, Lund T, Cabot W. A lagrangian dynamic subgrid-scale model of turbulence. J Fluid Mech 1996;319:353.

[93] Porte-Aget F, Meneveau C, Parlange M. A dynamic scale dependent model for large eddy simulation: application to the atmospheric boundary layer. J Fluid Mech 2000;415:261-84.

[94] Schumann U. Realizability of Reynolds-stress turbulence models. Phys Fluids $1977 ; 20(5)$

[95] Speziale CG. Invariance of Reynolds stress models. Phys Fluids 1979;22:1033.

[96] Vreman B, Geurts B, Kuerten H. On the formulation of the dynamic mixed subgrid-scale model. Phys Fluids 1994;6(12):4057-9.
[97] Ducros F, Nicoud F, Poinsot T. Wall-adapating local eddy-viscosity models for simulations in complex geometries. In: Baines MJ, editor. ICFD; 1998. p. 293-300.

[98] Vreman AW. An eddy-viscosity subgrid-scale model for turbulent shear flow: algebraic theory and applications. Phys Fluids 2004;16(10):3670-81. http://dx.doi.org/10.1063/1.1785131

[99] Kalitzin G, Templeton JA, Medic G. A near-wall eddy-viscosity formulation for LES. In: CY-Les 2005 Limassol; 2005. Cyprus.

[100] Nicoud F, Hubert Baya Toda H, Cabrit O, Bose S, Lee J. Using singular values to build a subgrid-scale model for large eddy simulations. Phys Fluids 2011; 23(085106).

[101] Gatski TB, Speziale CG. On explicit algebraic stress models for complex turbulent flows. J Fluid Mech 1993;254:59-78.

[102] Piomelli U. High Reynolds number calculations using the dynamic subgridscale stress model. Phys Fluids A 1993;5:1484-90.

[103] Piomelli U, Balaras E. Wall-layer models for large-eddy simulations. Ann Rev Fluid Mech 2002;34:349-74. http://dx.doi.org/10.1146/annurev.fluid.34. 082901.144919.

[104] Piomelli U, Balaras E, Pasinato H, Squires KD, Spalart PR. The inner-outer layer interface in large-eddy simulations with wall-layer models. Int J Heat Fluid Flow 2003;24(4):538-50. http://dx.doi.org/10.1016/S0142-727X(03) 00048-1.

[105] Piomelli U. Wall-layer models for large-eddy simulations. Prog Aerospace Sc 2008;44(6):437-46. http://dx.doi.org/10.1016/j.paerosci.2008.06.001

[106] Bardina J, Ferziger JH, Reynolds WC. Improved subgrid scale models for large-eddy simulations. Am Inst Aeronaut Astronaut J 1980;34:1111-9.

[107] Clark R, Ferziger J, Reynolds W. Evaluation of subgrid models using an accurately simulated flow. J Fluid Mech 1979;91:1-16.

[108] Adams N, Stolz S. Deconvolution methods dor subgrid-scale approximations in les. In: Geurts B, editor. Modern simulation strategies for turbulent flow. Edwards; 2001. p. 21-44.

[109] Zang Y, Street RL, Koseff JR. A dynamic subgrid-scale model and its application to turbulent recirculating flows. Phys Fluids 1993;5(12): 3186-96.

[110] Lodato G, Domingo P, Vervisch L. Three-dimensional boundary conditions for direct and large-eddy simulation of compressible viscous flow. J Comput Phys 2008.

[111] Porter D, Pouquet A, Woodward P. Kolmogorov-like spectra in decaying three-dimensional supersonic flows. Phys Fluids 1994;6:2133-42.

[112] Misra A, Pullin D. A vortex-based subgrid stress model for large-eddy simulation. Phys Fluids 1997:9:2443-54.

[113] Gicquel L, Givi P, Jaberi F, Pope S. Velocity filtered density function for large eddy simulation of turbulent flows. Phys Fluids 2002;14:1196-213.

[114] Fureby C, Grinstein F. Large eddy simulation of high-Reynolds-number free and wall-bounded flows. J Comput Phys 2002;181:68-97.

[115] Layton WJ. A mathematical introduction to large eddy simulations. Tech Rep.. URL: University of Pittsburg. Department of Mathematics http://www. mathematics.pitt.edu/research/technical-reports.php; 2002.

[116] Geurts B, Holm D. Leray and ns-alpha modeling of turbulent mixing. J Turb 2006;10.

[117] Farhat C, Rajasekharan A, Koobus B. A dynamic variational multiscale method for large eddy simulations on unstructured meshes. Comput Meth Appl Mech Eng 2006;195(13-16):1667-91.

[118] Mathey F, Chollet JP. Subgrid-scale model of scalar mixing for large eddy simulation of turbulent flows. In: Chollet JP, Voke PR, Kleiser L, editors. Direct and large eddy simulation II. Kluwer Academic Publishers; 1997. p. 103-14.

[119] Pope SB. Stochastic lagrangian models of velocity in homogeneous turbulent shear flow. Phys Fluids 2002;14(5):1696-702.

[120] Batchelor GK. An introduction to fluid dynamics. Cambridge: Cambridge University Press; 1988.

[121] Wong VC, Lilly DK. A comparison of two dynamic subgrid closure methods for turbulent thermal convection. Phys Fluids 1994;6(2):1016-23. http://dx. doi.org/10.1063/1.868335

[122] Wang W, Pletcher RH. On the large eddy simulation of a turbulent channe flow with significant heat transfer. Phys Fluids 1996;8(12):3354-66. http:// dx.doi.org/10.1063/1.869110.

[123] Wang BC, Yee E, Bergstrom DJ, Iida O. New dynamic subgrid-scale heat flux models for large-eddy simulation of thermal convection based on the general gradient diffusion hypothesis. J Fluid Mech 2008;604:125-63. http://dx.doi.org/10.1017/S0022112008001079.

[124] You D, Moin P. A dynamic global-coefficient subgrid-scale model for largeeddy simulation of turbulent scalar transport in complex geometries. Phys Fluids 2009;21(4):045109. http://dx.doi.org/10.1063/1.3115068.

[125] Kerstein AR. A linear eddy model of turbulent scalar transport and mixing. Combust Sci Tech 1988;60:391.

[126] Kerstein AR. Linear-eddy modeling of turbulent transport: part ii. Application to shear layer mixing. Combust Flame 1989;75(3-4):397-413.

[127] Kerstein AR. Linear-eddy modelling of turbulent transport. Part 3. Mixing and differential molecular diffusion in round jets. J Fluid Mech 1990;216: 411-35.

[128] Kerstein AR. Linear-eddy modelling of turbulent transport. Part 6. Microstructure of diffusive scalar mixing fields. J Fluid Mech 1991:231:361-94.

[129] McMurthy PA, Menon S, Kerstein AR. A linear eddy subgrid model for turbulent reacting flows: application to hydrogen-air combustion. Proc Combust Inst 1992;24(1):271-8. 
[130] Kerstein AR. Linear eddy modeling of turbulent transport. Part 4: structure of diffusion flames. Combust Sci Tech 1992;81:75-96.

[131] McMurthy PA, Gansauge TC, Kerstein AR, Krueger SK. Linear eddy simulations of mixing in a homogeneous turbulent flow. Phys Fluids A 1993;5(4): 1023-34.

[132] Menon S, McMurthy PA, Kerstein AR. A linear eddy mixing model for large eddy simulation of turbulent combustion. In: Galperin B, Orzag S, editors. Large eddy simulation of complex engineering and geophysical flows. Cambridge University Press; 1993. p. 87-314.

[133] Menon S, McMurtry PA, Kerstein AR. A linear eddy subgrid model for turbulent combustion: application to premixed combustion. In: 93-0107, A., editor. 31st Aerospace Sciences Meeting and Exhibit. Reno, NV, 1993.

[134] Sheikhi M, Drozda T, Givi P, Jaberi F, Pope S. Large eddy simulation of a turbulent nonpremixed piloted methane jet flame (sandia flame d). Proc Combust Inst 2005;20:549-56.

[135] Sheikhi M, Givi P, Pope S. Velocity-scalar filtered mass density function for large eddy simulation of turbulent reacting flows. Phys Fluids 2007; 9(095106):19.

[136] Peters N, Rogg B. Reduced kinetic mechanisms for applications in combustion systems. Lecture notes in physics. Heidelberg: Springer Verlag; 1993.

[137] Echeckki T, Mastorakos E. Advances, new trends and perspectives. In: Echeckki T, Mastorakos E, editors. Turbulent combustion modeling. Fluid mechanics and its applications, vol. 95. Springer; 2011.

[138] Cook AW, Riley JJ. Subgrid scale modeling for turbulent reacting flows. Combust Flame 1998;112:593-606.

[139] Candel S, Thevenin D, Darabiha N, Veynante D. Progress in numerical combustion. Combust Sci Technol 1999;149:297-337.

[140] Bilger R. Future progress in turbulent combustion research. Prog Energy Comb Sci 2000;26:367-80.

[141] Branley N, Jones W. Large eddy simulation of turbulent flames. In: European congress on computational methods in applied science and engineering; 2000. p. 1-23. Barcelona, Spain.

[142] Menon S. Subgrid combustion modelling for large-eddy simulations. Int J Engine Res 2000;1:209-27.

[143] Givi P. A review of modern developments in large eddy simulation of turbulent reactive flows. In: DNS/LES Progress and Challenges. Proceedings of the Third AFOSR International Conference on DNS/LES; ADP013626. Defense Technical Information Center Compilation Part Notice, 2001.

[144] Janicka J, Sadiki A. Large eddy simulation for turbulent combustion. Proc Combust Inst 2004;30:537-47.

[145] Lam S, Goussis D. Understanding complex chemical kinetics with computational singular perturbation. Proc Combust Inst 1988:931-41.

[146] Maas U, Pope SB. Implementation of simplified chemical kinetics based on low-dimensional manifolds. Proc Combust Inst 1992;24:719-29.

[147] Maas U, Pope SB. Simplifying chemical kinetics: intrinsic low-dimensional manifolds in composition space. Combust Flame 1992;88:239-64.

[148] Brown P, Byrne G, Hindmarsh A. Vode: a variable coefficient ode solver. SIAM J Sci Comput 1989;10:1038-51.

[149] Petzold L. A descriotion of dassl: a differential/algebrais system solver. Tech. Rep. SAND82-8637. Sandia National Laboratories; 1982.

[150] van Oijen JA, Lammers FA, de Goey LPH. Modeling of premixed laminar flames using flamelet generated manifolds. Combust Sci Tech 2001;127: 2124-34.

[151] de Goey LPH, van Oijen JA, Bongers H, Groot GRA. New flamelet based reduction methods: the bridge between chemical reduction techniques and flamelet methods. In: European combustion meeting; 2003. Orléans (France).

[152] Gicquel O, Darabiha N, Thévenin D. Laminar premixed hydrogen/air counterflow flame simulations using flame prolongation of ildm with differential diffusion. Proc Combust Inst 2000;28:1901-8.

[153] Fiorina B, Baron R, Gicquel O, Thévenin D, Carpentier S, Darabiha N. Modelling non-adiabatic partially premixed flames using flame-prolongation of ildm. Combust Theory Model 2003;7:449-70.

[154] Fiorina B, Gicquel O, Vervisch L, Carpentier S, Darabiha N. Premixed turbulent combustion modeling using a tabulated detailed chemistry and pdf. Proc Combust Inst 2005;30(1):867-74.

[155] Fiorina B, Gicquel O, Vervisch L, Carpentier S, Darabiha N. Approximating the chemical structure of partially-premixed and diffusion counter-flow flames using FPI flamelet tabulation. Combust Flame 2005:140:147-60.

[156] Gicquel O. Développement de méthodes automatiques de réduction de schémas cinétiques dédiées à la combustion laminaire et turbulente. Habilitation à diriger des recherches; 2005.

[157] Pope SB. Computationally efficient implementation of combustion chemistry using in situ adaptive tabulation. Combust Theory Model 1997;1:41-63.

[158] Tonse S, Moriary N, Brown N, Frenklach M. Prism: piece reusable implementation of solution mapping. an economical strategy for chemical kinetics. Israel J Chem 1999;39:97-106.

[159] Christo F, Masri A, Nebot E. An integrated pdf/neural network approach for simulating turbulent reacting systems. Proc Combust Inst 1996;27: 1793-807.

[160] Searby G, Quinard J. Direct and indirect measurements of markstein numbers of premixed flames. Combust Flame 1990;82:298-311.

[161] Bradley D, Gaskell PH, Gu XJ. Burning velocities, markstein lengths, and flame quenching for spherical methane-air flames: a computational study. Combust Flame 1996;104(1/2):176-98.
[162] Gu XJ, Haq MZ, Lawes M, Woolley R. Laminar burning velocity and markstein lengths of methane-air mixtures. Combust Flame 2000;121:41-58.

[163] Zeldovich YB, Frank-Kamenetskii DA. Selected works of Ya. B. Zeldovich, chemical physics and hydrodynamics. Princeton University Press; 1962.

[164] Hartley L, Dold JW. Flame propagation in a nonuniform mixture: analysis of a triple flame. Combust Sci Tech 1991;80:23.

[165] Kioni PN, Rogg B, Bray KNC, Li nán A. Flame spread in laminar mixing layers: the triple flame. Combust Flame 1993;95:276.

[166] Veynante D, Duclos J, Piana J. Experimental analysis of flamelet models for premixed turbulent combustion. Proc Combust Inst 1994;25(1): 1249-56.

[167] Ruetsch G, Veynante D, Vervisch L, Poinsot T, Li nán A. Triple flame structure and diffusion flame stabilization. In: APS meeting; 1994. Atlanta.

[168] Ruetsch GR, Vervisch L, Li nán A. Effects of heat release on triple flames. Phys Fluids 1995;7(6):1447-54.

[169] Domingo P, Vervisch L. Triple flames and partially premixed combustion in autoignition of non-premixed mixtures. Proc Combust Inst 1996;26(1): 233-40.

[170] Kioni PN, Bray KNC, Greenhalgh DA, Rogg B. Experimental and numerical study of a triple flame. Combust Flame 1998;116:192-206.

[171] Ghosal S, Vervisch L. Theoretical and numerical investigation of a symmetrical triple flame using a parabolic flame type approximation. J Fluid Mech 2000;415:227-60.

[172] Veynante D, Poinsot T. Reynolds averaged and large eddy simulation modeling for turbulent combustion. In: Metais O, Ferziger J, editors. New tools in turbulence modelling. Lecture 5. Les editions de Physique. Springer; 1997. p. $105-35$.

[173] Broadwell JE, Mungal MG. Molecular mixing and chemical reactions in turbulent shear layers. Proc Combust Inst 1989;22(1):579-87.

[174] Cant RS, Bray KNC. Strained laminar flamelet calculations of premixed turbulent combustion in a closed vessel. Proc Combust Inst 1989;22(1): 791-9.

[175] Ashurst WT, McMurtry PA. Flame generation of vorticity: vortex dipoles from monopoles. Combust Sci Tech 1989;66:17-37.

[176] Candel S, Veynante D, Lacas F, Maistret E, Darabiha N, Poinsot T. Coherent flame model: applications and recent extensions. In: Larrouturou BE, editor. Advances in combustion modeling. Series on advances in mathematics for applied sciences. Singapore: World Scientific; 1990. p. 19-64.

[177] Cant RS, Pope SB, Bray KNC. Modelling of flamelet surface to volume ratio in turbulent premixed combustion. Proc Combust Inst 1991;23(1):809-15.

[178] Cant RS, Rutland CJ, Trouvé A. Statistics for laminar flamelet modeling. In: Proc. of the summer program. Center for Turbulence Research, Stanford Univ./NASA-Ames; 1990. p. 271-9.

[179] Gao F. A large-eddy simulation scheme for turbulent reacting flows. Phys Fluids 1993;5(6):1282-4.

[180] Pope SB. Self-conditioned fields for large-eddy simulations of turbulent flows. J Fluid Mech 2010;652:139-69.

[181] Navarro-Martinez S, Kronenburg A. Les-cmc simulations of a turbulent bluffbody flame. Proc Combust Inst 2007;31:1721-8.

[182] Colucci P, Jaberi F, Givi P, Pope S. Filtered density function for large eddy simulation of turbulent reacting flows. Phys Fluids 1998;10:499-515.

[183] Jaberi F, Colucci P, James S, Givi P, Pope S. Filtered mass density function for large-eddy simulation of turbulent reacting flows. J Fluid Mech 1999;401: 85-121.

[184] Drozda TG, Sheikhi MRH, Madnia CK, Givi P. Developments in formulation and application of the filtered density function. Flow Turb Combust 2007;78: 35-67.

[185] Sheikhi MRH, Givi P, Pope S. Frequency-velocity-scalar filtered mass density function for large eddy simulation of turbulent flows. Phys Fluids 2009; 21(075102).

[186] Pope SB. Computations of turbulent combustion: progress and challenges. Proc Combust Inst 1991;23(1):591-612.

[187] Candel S. Combustion. Ecole d'été d'analyse numérique EDF-CEA-INRIA, 1993.

[188] Meneveau C, Lund T. The dynamic smagorinsky model and scale-dependent coefficients in the viscous range of turbulence. Phys Fluids 1997;9(12): 3932-4.

[189] Fiorina B, Gicquel O, Veynante D. Turbulent flame simulation taking advantage of tabulated chemistry self-similar properties. Proc Combust Inst 2009;32:1687-94

[190] Fiorina B, Vicquelin R, Auzillon P, Darabiha N, Gicquel O, Veynante D. A filtered tabulated chemistry model for LES of premixed combustion. Combust Flame 2010;157(3):465-75.

[191] Kemenov K, Pope S. Molecular diffusion effects in les of a piloted methaneair flame. Combust Flame 2011;158(2):240-54.

[192] Moureau V, Domingo P, Vervisch L. From large-eddy simulation to direct simulation of a lean premixed swirl flame: filtered laminar flame-pdf modeling. Combust Flame 2011a. http://dx.doi.org/10.1016/ j.combustflame.2010.12.004.

[193] Geurts BJ, Frohlich J. A framework for predicting accuracy limitations in large-eddy simulations. Phys Fluids 2002;14(6):L41-4.

[194] Meyers J, Geurts BJ, Baelmans M. Database analysis of errors in large-eddy simulation. Phys Fluids 2003;15(9):2740-55.

[195] Meyers J, Sagaut P. Is plane-channel flow a friendly case for the testing of large-eddy simulation subgrid-scale models? Phys Fluids 2007;19:1-4. 
[196] Lucor D, Meyers J, Sagaut P. Sensitivity analysis of large-eddy simulations to subgrid-scale-model parametric uncertainty using polynomial chaos. J Fluid Mech 2007;585:255-79.

[197] Pope SB. Ten questions concerning the large-eddy simulation of turbulent flows. New J Phys 2004;6:35.

[198] Celik I, Cehreli A, Yavuz I. Index of resolution quality for large eddy simulations. J Fluids Eng 2005;127:949-58.

[199] Klein M. An attempt to assess the quality of large eddy simulations in the context of implicit filtering. Flow Turb Combust 2005;75(1-4):131-47.

[200] Vervishc L, Domingo P, Lodato G, Veynante D. Scalar energy fluctuations in large-eddy simulation of turbument flames: statistical budgets and mesh quality criterion. Combust Flame 2010;157(4):778-89.

[201] Gourdain N, Gicquel L, Montagnac M, Vermorel O, Gazaix M, Staffelbach G et al. High performance parallel computing of flows in complex geometries part 1: methods. Comput Sci Discov 2009;2(015003):26.

[202] Gourdain N, Gicquel L, Staffelbach G, Vermorel O, Duchaine F, Boussuge JF, et al. High performance parallel computing of flows in complex geometries part 2: applications. Comput Sci Discov 2009;2(015004):28

[203] Colin O, Rudgyard M. Development of high-order taylor-galerkin schemes for unsteady calculations. J Comput Phys 2000;162(2):338-71.

[204] Grcar J, Bell J, Day M. The soret effect in naturally propagating, premixed, lean, hydrogen-air flames. Proc Combust Inst 2009;32(1173).

[205] Moureau V, Domingo P, Vervisch L. Design of a massively parallel cfd code for complex geometries. Comptes Rendus Mecanique 2011b. http:/ dx.doi.org/10.1016/j.crme.2010.12.001.

[206] Chen JH. Petascale direct numerical simulation of turbulent combustion fundamental insights towards predictive models. Proc Combust Inst 2011; 33(1):99-123.

[207] Gicquel L, Gourdain N, Boussuge JF, Deniau H, Staffelbach G, Wolf P, et al. High performance parallel computing of flows in complex geometries. Comptes Rendus Mecanique 2011;339:104-24.

[208] Syred N, Beer J. Combustion in swirling flows: a review. Combust Flame 1974;23:143-201.

[209] Sloan DG, Smith PJ, Smoot LD. Modeling of swirl in turbulent flow systems. Prog Energy Comb Sci 1986;12:163-250.

[210] Gupta A, Beer J, Swithenbank J. Concentric multi-annular swirl burner: stability limits and emission characteristics. Proc Combust Inst 1977;16: 79-91.

[211] Weber R, Visser B, Boysan F. Assessment of turbulent modeling for engineering prediction of swirling vortices in the near burner zone. Int $\mathrm{J}$ Heat Fluid Flow 1990;11:225-38.

[212] Kitoh O. Experimental study of turbulent swirling flow in a straight pipe. J Fluid Mech 1991;225:445-65.

[213] Ahmed S. Velocity measurements and turbulence statistics of a confined isothermal swirling flow. Exp Therm Fluid Sci 1998;17:256-70.

[214] Nathan G, Turns Hill S, Luxton R. An axisymmetric fluidic nozzle to generate jet precession. J Fluid Mech 1998;370:347-80.

[215] Al-Abdeli Y, Masri A. Recirculation and flow field regimes of unconfined nonreacting swirling flow. Exp Therm Fluid Sci 2003;23:655-65.

[216] Masri AR, Kalt P, Barlow RS. The compositional structure of swirl-stabilised turbulent nonpremixed flames. Combust Flame 2004;137:1-37.

[217] Gentemann A, Hirsch C, Kunze K, Kiesewetter F, Sattelmayer T, Polifke W. Validation of flame transfer function reconstruction for perfectly premixed swirl flames. In: ASME, editor. ASME Turbo Expo. Vienna, Austria: ASME; 2004, p. Paper GT2004-53776.

[218] Hirsch C, Fanaca D, Alemela D, Pölifke R, Sattelmayer W. Influence of the swirler design on the flame transfer function of premixed flames. In: ASME, editor. Proceedings of AMSE Turbo Expo: Power for Land, Sea and Air; Pape GT2005-68195. Nevada, USA: ASME Turbo-Expo; 2005.

[219] Palies P, Durox D, Schuller T, Morenton P, Candel S. Dynamics of premixed confined swirling flames. Comptes Rendus Mecanique 2009;337:395-405.

[220] Komarek T, Polifke W. Impact of swirl fluctuations on the flame response of a perfectly premixed swirl burner. J Eng Gas Turb Power 2010;132(6) 061503.

[221] Palies P, Durox D, Schuller T, Candel S. The combined dynamics of swirler and turbulent premixed swirling flames. Combust Flame 2010;157:1698-717.

[222] Sarpkaya T. On stationary and travelling vortex breakdowns. J Fluid Mech $1971 ; 45: 545-59$

[223] Escudier MP, Zehnder N. Vortex-flow regimes. J Fluid Mech 1982;115: 105-21.

[224] Gupta AK, Lilley DG, Syred N. Swirl flows. Abacus Press; 1984

[225] Escudier M. Vortex breakdown: observations and explanations. Prog Aerospace Sci 1988;25:189-229.

[226] Lucca-Negro O, O’Doherty T. Vortex breakdown: a review. Prog Energy Comb Sci 2001;27:431-81.

[227] Novak F, Sarpkaya T. Turbulent vortex breakdown at high Reynolds numbers. Am Inst Aeronaut Astronaut J 2000;38:825-34.

[228] Syred N. A review of oscillation mechanisms and the role of the processing vortex core in swirl combustion systems. Prog Energy Comb Sci 2006;32(2): 93-161.

[229] Pierce CD, Moin P. A dynamic model for subgrid scale variance and dissipation rate of a conserved scalar. Phys Fluids 1998;10(12):3041-4.

[230] Wang P, Bai X, Wessman M, Kingmann J. Large eddy simulation and experimental studies of a confined turbulent swirling flow. Phys Fluids 2004: 16:3306-24.
[231] Kempf AM, Janicka J, Lindstedt R. Large eddy simulation of a bluff body stabilized non-premixed flame. Combust Flame 2006;144:170-89.

[232] Malalasekera W, Ibrahim SS, Rang Dinesh KKJ, Kirkpatrick MP. Large eddy simulation of isothermal turbulent swirling jets. Combust Sci Tech 2007; 179(8):1481-525.

[233] Stein O, Kempf AM, Janicka J. Les of the Sydney swirl flame series: an initia investigation of the fluid dynamics. Combust Sci Technol 2007;179(1 \& 2): 173-89.

[234] Syred N, Dahmen K. The effect of high levels of confinement upon the aerodynamics of swirl burners. AIAA J Energy 1978;2(1):8-15.

[235] Midgley K, Spencer A, McGuirk J. Unsteady flow structures in radial swirler fed fuel injectors. In: ASME, editor. Proceedings of AMSE turbo expo: power for land, sea and air; 2004. Vienna, Austria.

[236] Midgley K, Spencer A, McGuirk J. Unsteady flow structures in radial swirler fed fuel injectors. J Eng Gas Turb Power 2005;127(4):755-65.

[237] Spencer A, McGuirk J. Vortex breakdown in swirling fuel injector flows. J Eng Gas Turb Power 2008;130(2):021503-11.

[238] Valera-Medina A, Syred N, Griffiths A. Visualisation of isothermal large coherent structures in a swirl burner. Combust Flame 2009;156:1723-34.

[239] Janus B, Dreizler A, Janicka J. Flow field and structure of swirl stabilized nonpremixed natural gas flames at elevated pressure. In: ASME, editor. Proceedings of ASME turbo expo: power for land, sea and air. Vienna, Austria: ASME; 2004

[240] Janus B, Dreizler A, Janicka J. Experimental study on stabilization of lifted flames in a model GT combustor. Flow Turb Combust 2004;75:293-315.

[241] Wegner B, Kempf A, Schneider C, Sadiki A, Schafer M. Large eddy simulation of combustion processes under gas turbine conditions. Prog Comput Fluid Dynam Int J 2004;(3/4/5):257-63.

[242] Wang S, Hsieh S, Yang V. Unsteady flow evolution in swirl injector with radial entry. i. Stationary conditions. Phys Fluids 2005;17:045106.

[243] Wang S, Yang V. Unsteady flow evolution in swirl injector with radial entry. ii. External excitations. Phys Fluids 2005;17:045107.

[244] Wang S, Yang V, Hsiao G, Mongia H. Large eddy simulation of gas turbine swirl injector flow dynamics. J Fluid Mech 2007;583:99-122.

[245] Al-Abdeli Y, Masri AR. Stability characteristics and flow-fields of turbulent non-premixed swirling flames. Combust Theory Model 2003;7:731-66.

[246] Selle L, Lartigue G, Poinsot T, Koch R, Schildmacher KU, Krebs W, et al Compressible large-eddy simulation of turbulent combustion in complex geometry on unstructured meshes. Combust Flame 2004;137(4): 489-505.

[247] Roux S, Lartigue G, Poinsot T, Meier U, Bérat C. Studies of mean and unsteady flow in a swirled combustor using experiments, acoustic analysis and large eddy simulations. Combust Flame 2005;141:40-54.

[248] Wang LP, Wexler AS, Zhou Y. Statistical mechanical description and modelling of turbulent collision of inertial particles. J Fluid Mech 2000;415: 117-53.

[249] Wang M, Moin P. Dynamic wall modeling for large-eddy simulation of complex turbulent flows. Phys Fluids 2002;14(7):2043-51. http://dx.doi org/10.1063/1.1476668.

[250] Huang Y, Yang V. Dynamics and stability of lean-premixed swirl-stabilized combustion. Prog Energy Comb Sci 2009;35:293-364.

[251] Lu X, Wang S, Sung HG, Hsieh SY, Yang V. Large-eddy simulations of turbulent swirling flows injected into a dump chamber. J Fluid Mech 2005; 527:171-95.

[252] Wegner B, Maltsev A, Schneider C, Sadiki A, Dreizler A, Janicka J. Assessment of unsteady rans in predicting swirl flow instability based on les and experiments. Int J Heat Fluid Flow 2004;25:528-36.

[253] Stohr M, Sadanandan R, Meier W. Experimental study of unsteady flame structures of an oscillating swirl flame in a gas turbine model combustor. Proc Combust Inst 2009;32:2925-32.

[254] Ranga Dinesh K, Kirkpatrick MP. Study of jet precession, recirculation and vortex breakdown in turbulent swirling jets using les. Comput Fluids 2009; 38:1232-42.

[255] Ranga Dinesh K, Kirkpatrick MP, Jenkins K. Investigation of the influence of swirl on a confined coannular swirl jet. Comput Fluids 2010;39:756-67.

[256] TNF. Turbulent non-premixed flame series. URL: http://www.sandia.gov/ TNF/abstract.html; 1996

[257] Combustion Institute. Combustion symposium series. URL: http://www. journals.elsevier.com/proceedings-of-the-combustion-institute/; 1948.

[258] Kempf A. Les validation from experiments. Flow Turb Combust 2008;80: $351-73$.

[259] Branley N, Jones WP. Large eddy simulation of a turbulent non-premixed flame. Combust Flame 2001;127:1914-34.

[260] Forkel H, Janicka J. Large-eddy simulation of a turbulent hydrogen diffusion flame. Flow Turb Combust 2000;65(2):163-75.

[261] Franzelli B, Riber E, Sanjose M, Poinsot T. A two-step chemical scheme for kerosene-air premixed flames. Combust Flame 2010;157(7):1364-73.

[262] Légier JP, Varoquié B, Lacas F, Poinsot T, Veynante D. Large eddy simulation of a non-premixed turbulent burner using a dynamically thickened flame model. In: Pollard A, Candel S, editors. IUTAM symposium on turbulent mixing and combustion. Kluwer Academic Publishers; 2002. p. 315-26.

[263] Freitag M, Janicka J. Investigation of a strongly swirled premixed flame using les. Proc Combust Inst 2007;31:1477-85.

[264] Li G, Gutmark E. Boundary conditions effects on nonreacting and reacting flows in a multiswirl combustor. Am Inst Aeronaut Astronaut J 2006;44(3):444-56. 
[265] RIKEN. The development of the next-generation supercomputer. URL: http:// www.nsc.riken.jp/index-eng.html; 2006.

[266] Bergmann V, Meier W, Wolff D, Stricker W. Appl Phys B 1998:66:489.

[267] Meier W, Barlow RS, Chen YL, Chen JY. Ramman/Rayleigh/LIF measurements in a turbulent $\mathrm{ch} 4 / \mathrm{h} 2 / \mathrm{n} 2$ jet diffusion flame: experimental techniques and turbulence-chemistry interaction. Combust Flame 2000;123(3): 326-43.

[268] Schneider C, Dreizler A, Janicka J. Flow field measurements of stable and locally extinguishing hydrocarbon-fuelled jet flames. Combust Flame 2003; 135(1-2):185-90.

[269] Weigand P, Meier W, Duan X, Stricker W, Aigner M. Investigations of swirl flames in a gas turbine model combustor: I. flow field, structures, temperature, and species distributions. Combust Flame 2006;144(1-2):205-24

[270] Meier W, Duan X, Weigand P. Investigations of swirl flames in a gas turbine model combustor: I. flow field, structures, temperature, and species distributions. Combust Flame 2006;144(1-2):225-36.

[271] Meier W, Weigand P, Duan X, Giezendanner-Thoben R. Detailed characterization of the dynamics of thermoacoustic pulsations in a lean premixed swirl flame. Combust Flame 2007;150(1-2):2-26.

[272] Kaiser S, Frank J. Imaging of dissipative structures in the near-field of a turbulent non-premixed jet flame. Proc Combust Inst 2007;31:1515-23.

[273] Frank JH, Kaiser SA, Oefelein JC. Analysis of scalar mixing dynamics in les using high-resolution imaging of laser Rayleigh scattering in turbulent nonreacting jets and non-premixed jet flames. Proc Combust Inst 2011;33(1): 1373-81.

[274] Moureau V, Minot P, Pitsch H, Bérat C. A ghost-fluid method for large-eddy simulations of premixed combustion in complex geometries. J Comput Phys 2007;221(600-614)

[275] Galpin J, Naudin A, Vervisch L, Angelberger C, Colin O, Domingo P. Largeeddy simulation of a fuel-lean premixed turbulent swirl-burner. Combust Flame 2008;155(1-2):247-66.

[276] Albouze G, Poinsot T, Gicquel LYM. Chemical kinetics modelisation and les combustion model effects on a perfectly premixed burner. C R Acad Sci Mécanique 2009;337(6-7):318-28

[277] Franzelli B, Riber E, Gicquel L, Poinsot T. Large-Eddy Simulation of combustion instabilities in a lean partially premixed swirled flame. Combust Flame 2012;159(2):621-37.

[278] Yoo C, Sankaran R, Chen JH. Three-dimensional direct numerical simulation of a turbulent lifted hydrogen jet flame in heated coflow: flame stabilization and structure. J Fluid Mech 2010;640:453-81.

[279] Auzillon P, Fiorina B, Vicquelin R, Darabiha N, Gicquel O, Veynante D. Modeling chemical flame structure and combustion dynamics in les. Proc Combust Inst 2011;33(1):1331-8

[280] URL:Advisory council for aeronautics research in Europe. Towards 2050 http://www.acare4europe.com/; 2011

[281] United Nations. Framework convention on climate change. URL: http:// unfccc.int/kyoto_protocol/items/2830.php; 1997.

[282] Lieuwen T, Yang V. Combustion instabilities in gas turbine engines. Operational experience, fundamental mechanisms and modeling. In: Prog. in astronautics and aeronautics AIAA, vol. 210; 2005.

[283] Rayleigh L. The explanation of certain acoustic phenomena. Nature 1878 July; $18: 319-21$.

[284] Crocco L. Aspects of combustion instability in liquid propellant rocket motors. part i. J Am Rocket Soc 1951;21:163-78.

[285] Crocco L. Aspects of combustion instability in liquid propellant rocket motors. part ii. J Am Rocket Soc 1952;22:7-16.

[286] Crocco L, Cheng SI. Theory of combustion instability in liquid propellant rocket motors; vol. Agardograph no 8. Butterworths Science; 1956.

[287] Candel S, Huynh C, Poinsot T. Some modeling methods of combustion instabilities. In: Unsteady combustion; 1993. Praia da Granja.

[288] Candel S, Huynh C, Poinsot T. Some modeling methods of combustion instabilities. In: Unsteady combustion. Nato ASI series. Dordrecht: Kluwer Academic Publishers; 1996. p. 83-112.

[289] Poinsot T, Lele S. Boundary conditions for direct simulations of compressible viscous flows. J Comput Phys 1992;101(1):104-29. http://dx.doi.org/10. 1016/0021-9991(92)90046-2.

[290] Nicoud F. Defining wave amplitude in characteristic boundary conditions. J Comput Phys 1998;149(2):418-22.

[291] Kaufmann A Nicoud F, Poinsot T. Flow forcing techniques for numerical simulation of combustion instabilities. Combust Flame 2002;131:371-85.

[292] Selle L, Nicoud F, Poinsot T. The actual impedance of non-reflecting boundary conditions: implications for the computation of resonators. Am Inst Aeronaut Astronaut J 2004b;42(5):958-64.

[293] Martin C, Benoit L, Sommerer Y, Nicoud F, Poinsot T. LES and acoustic analysis of combustion instability in a staged turbulent swirled combustor. Am Inst Aeronaut Astronaut J 2006;44(4):741-50.

[294] Noiray N, Durox D, Schuller T, Candel S. A unified framework for nonlinear combustion instability analysis based on the flame describing function a unified framework for nonlinear combustion instability analysis based on the flame describing function. J Fluid Mech 2008;615:139-67.

[295] Benoit L, Nicoud F. Numerical assessment of thermo-acoustic instabilities in gas turbines. In: Baines MJ, editor. ICFD; 2004. Oxford.

[296] Giauque A, Selle L, Poinsot T, Buechner H, Kaufmann P, Krebs W. System identification of a large-scale swirled partially premixed combustor using LES and measurements. J Turb 2005;6(21):1-20.
[297] Sattelmayer T. Influence of the combustor aerodynamics on combustion instabilities from equivalence ratio fluctuations. J Eng Gas Turb Power 2003; 125:11-9.

[298] Lawn CJ, Evesque S, Polifke W. A model for the thermo-acoustic response of a premixed swirl burner: part i: acoustic aspects. Combust Sci Technol 2004; 176(8):1331-58.

[299] Lawn CJ, Polifke W. A model for the thermo-acoustic response of a premixed swirl burner: part ii: the flame response. Combust Sci Technol 2004;176(8): 1359-90.

[300] Huber A, Polifke W. Dynamics of practical premix flames, part i: model structure and identification. Int J Spray Combust Dynam 2009;1(2): 199-229.

[301] Tay Wo Chong L, Bomberg S, Ulhaq A, Kromarek T, Polifke W. Comparative validation study on identification of premixed flame transfer function. J Eng Gas Turbines Power 2011;134(\#2 021502):1-8.

[302] Palies P, Schuller T, Durox D, Candel S. Modelling of premixed swirling flames transfer functions. Proc Combust Inst 2011;33(2):2967-74.

[303] Sengissen A, Giauque A, Staffelbach G, Porta M, Krebs W, Kaufmann P, et al. Large eddy simulation of piloting effects on turbulent swirling flames. Proc Combust Inst 2007;31:1729-36.

[304] Sengissen A, Kampen JFV, Huls R, Stoffels G, Kok JBW, Poinsot T. Les and experimental studies of cold and reacting flows in a swirled partially premixed burner with and without fuel modulation. Combust Flame 2007;150: 40-53.

[305] Mastorakos E. Ignition of turbulent non-premixed flames. Prog Energy Comb Sci 2009;35(1):57-97.

[306] Boileau M, Mossa JB, Cuenot B, Poinsot T, Bissières D, Bérat C. Toward LES of an ignition sequence in a full helicopter combustor. URL: In: 1st workshop INCA. SNECMA, Villaroche, France. p. 27-34 http://www.cerfacs.fr/cfdbib/ repository/TR_CFD_05_72.pdf; 2005.

[307] Boileau M, Staffelbach G, Cuenot B, Poinsot T, Bérat C. LES of an ignition sequence in a gas turbine engine. URL: Combust Flame 2008;154(1-2):2-22 http://www.cerfacs.fr/cfdbib/repository/TR_CFD_07_112.pdf.

[308] Lacaze G, Richardson E, Poinsot TJ. Large eddy simulation of spark ignition in a turbulent methane jet. Combust Flame 2009;156(6):1993-2009.

[309] Lacaze G, Cuenot B, Poinsot TJ, Oschwald M. Large eddy simulation of laser ignition and compressible reacting flow in a rocket-like configuration. Combust Flame 2009;156(6):1166-80.

[310] Subramanian V, Domingo P, Vervisch L. Large-eddy simulation of forced ignition of an annular bluff-body burner. Combust Flame 2010;157(3): $579-601$.

[311] Weckering J, Sadiki A, Janicka J, Mastorakos E, Eggels R. A forced ignition probability analysis using les and lagrangian particle. Proc Combust Inst 2011;33(2):2919-25

[312] Jones WP, Prasad VN. Les-pdf simulation of a spark ignited turbulent methane jet. Proc Combust Inst 2011;33(1):1355-63.

[313] Cuenot B, Eyssartier A, Gicquel L, Poinsot T. Prediction of ignition probability of turbulent two-phase flames in industrial burners. Combust Flame, 2011, submitted.

[314] Ahmed SF, Balachandran R, Marchione T, Mastorakos E. Spark ignition of turbulent nonpremixed bluff-body flames. Combust Flame 2007;151: 366-85.

[315] Colin O, Truffin K. A spark ignition model for large eddy simulation based on an FSD transport equation (issim-les). Proc Combust Inst 2011:33(2):3097-104.

[316] Mare FD, Jones WP, Menzies K. Large eddy simulation of a model gas turbine combustor. Combust Flame 2004;137:278-95.

[317] Mahesh K, Constantinescu G, Apte S, laccarino G, Ham F, Moin P. Large eddy simulation of reacting turbulent flows in complex geometries. ASME J Appl Mech 2006;73:374-81.

[318] Moin P, Apte SV. Large-eddy simulation of realistic gas turbine combustors. Am Inst Aeronaut Astronaut J 2006;44(4):698-708.

[319] Boudier G, Gicquel LYM, Poinsot T, Bissières D, Bérat C. Comparison of LES, RANS and experiments in an aeronautical gas turbine combustion chamber. Proc Combust Inst 2007;31:3075-82.

[320] Boudier G, Gicquel LYM, Poinsot T, Bissières D, Bérat C. Effect of mesh resolution on large eddy simulation of reacting flows in complex geometry combustors. Combust Flame 2008;155(1-2):196-214.

[321] Mendez S, Nicoud F. Large-eddy simulation of a bi-periodic turbulent flow with effusion. J Fluid Mech 2008:598:27-65.

[322] Mendez S, Nicoud F. Adiabatic homogeneous model for flow around a multiperforated plate. Am Inst Aeronaut Astronaut J 2008;46(10):2623-33.

[323] Mendez S, Eldredge J. Acoustic modeling of perforated plates with bias flow for large-eddy simulations. J Comput Phys 2009;228(13):4757-72.

[324] Massot M, Villedieu P. Modélisation multi-fluide eulérienne pour la simulation de brouillards denses polydispersés. C R Acad Sci Problèmes mathématiques de la mécanique 2001;332(9):869-74.

[325] Laurent F, Massot M, Villedieu P. Eulerian multi-fluid modeling for the numerical simulation of coalescence in polydisperse dense liquid sprays. J Comput Phys 2004;194(2):505-43.

[326] Février P, Simonin O, Squires K. Partitioning of particle velocities in gas-solid turbulent flows into a continuous field and a spatially uncorrelated random distribution: theoretical formalism and numerical study. J Fluid Mech 2005; 533:1-46

[327] Sirignano WA. Volume averaging for the analysis of turbulent spray flows. Int J Multiphase Flow 2005;31(6):675-705. 
[328] Riber E, García M, Moureau V, Pitsch H, Simonin O, Poinsot T. Evaluation of numerical strategies for LES of two-phase reacting flows. J Comput Phys 2009;228(2):539-64.

[329] Apte SV, Mahesh K, Moin P, Oefelein JC. Large-eddy simulation of swirling particle-laden flows in a coaxial-jet combustor. Int J Multiphase Flow 2003; 29(8):1311-31.

[330] Réveillon J, Pera C, Massot M, Knikker R. Eulerian analysis of the dispersion of evaporating polydispersed sprays in a statistically stationary turbulent flow. J Turb 2004;5(1):1-27.

[331] Lefebvre AH. Atomization and sprays. Combustion. (Hemisphere Publishing Corporation). Taylor \& Francis; 1989

[332] Duchaine F, Corpron A, Pons L, Moureau V, Nicoud F, Poinsot T. Development and assessment of a coupled strategy for conjugate heat transfer with large eddy simulation: application to a cooled turbine blade. Int J Heat Fluid Flow 2009;30:1129-41.

[333] Duchaine F, Mendez S, Nicoud F, Corpron A, Moureau V, Poinsot T. Conjugate heat transfer with large eddy simulation application to gas turbine components. C R Acad Sci Mécanique 2009;337(6-7):550-61.

[334] Amaya J, Cabrit O, Poitou D, Cuenot B, El Hafi M. Unsteady coupling of Navier-Stokes and radiative heat transfer solvers applied to an anisothermal multicomponent turbulent channel flow. J Quant Spec Rad Transf 2010; 111(2):295-301. http://dx.doi.org/10.1016/j.jqsrt.2009.06.014.

[335] Wang Y. Direct numerical simulation of non-premixed combustion with soot and thermal radiation. Phd thesis, University of Maryland, 2005.

[336] Lefebvre AH. Gas turbines combustion. Taylor \& Francis; 1999.

[337] Fureby C, Grinstein F, Li G, Gutmark E. An experimental and computational study of a multi-swirl gas turbine combustor. Proc Combust Inst 2007;31(2): 3107-14.

[338] Roux A. Simulation aux grandes échelles d'un statoréacteur. Ph.D. thesis Université de Toulouse - Ecole doctorale MEGeP; CERFACS - CFD Team, Toulouse; 2009.

[339] Roux A, Gicquel LYM, Reichstadt S, Bertier N, Staffelbach G, Vuillot F, et al Analysis of unsteady reacting flows and impact of chemistry description in large eddy simulations of side-dump ramjet combustors. Combust Flame 2010;157:176-91.

[340] Leyko M, Nicoud F, Moreau S, Poinsot T. Massively parallel les of azimuthal thermo-acoustic instabilities in annular gas turbines. C R Acad Sci Mécanique 2009;337(6-7):415-25.

[341] Leyko M, Nicoud F, Poinsot T. Comparison of direct and indirect combustion noise mechanisms in a model combustor. Am Inst Aeronaut Astronaut 2009;47(11):2709-16. http://dx.doi.org/10.2514/1.43729.

[342] Wolf P, Balakrishnan R, Staffelbach G, Gicquel L, Poinsot T. Using LES to study reacting flows and instabilities in annular combustion chambers. Flow Turb Combust 2012;88(1-2):191-206.

[343] Stow SR, Zedda M, Triantafyllidis A, Garmory A, Mastorakos E, Mosbach T. Conditional moment closure les modelling of an aero-engine combustor at relight conditions. In: AMSE, editor. Proceedings of ASME turbo expo 2011. ASME; 2011. p. GT2011-45100.

[344] Triantafyllidis A, Mastorakos E, Eggels R. Large eddy simulations of forced ignition of a non-premixed bluff-body methane flame with conditional moment closure. Combust Flame 2009;156:2328-45.

[345] Polifke W, Paschereit CO. Determination of thermo-acoustic transfer matrices by experiment and computational fluid dynamics. ERCOFTAC Bulletin 1998:38.

[346] Schuermans B, Polifke W, Paschereit CO. Modeling transfer matrices of premixed flames and comparison with experimental results. In: International gas turbine \& aeroengine congress \& exposition. Indianapolis/Indiana: ASME Paper; 1999

[347] Nicoud F, Benoit L. Global tools for thermo-acoustic instabilities in gas turbines. In: APS/DFD meeting. Bull. Amer. Phys. Soc, vol. 48; 2003. New York.

[348] Benoit L, Nicoud F. Numerical assessment of thermo-acoustic instabilities in gas turbines. Int J Numer Meth Fluids 2005:47(8-9):849-55.

[349] Staffelbach G, Gicquel LYM, Poinsot T. Highly parallel large eddy simulation of multiburner configurations in industrial gas turbines. The Cyprus international symposium on complex effects in large eddy simulation; 2005.

[350] Polifke W, Poncet A, Paschereit CO, Doebbeling K. Reconstruction of acoustic transfer matrices by instationary computational fluid dynamics. J Sound Vib 2001;245(3):483-510.

[351] Kaess R, Polifke W, Poinsot T, Noiray N, Durox D, Schuller T, et al. Cfd-based mapping of the thermo-acoustic stability of a laminar premix burner. In Proc. of the summer program. USA: Center for Turbulence Research, NASA AMES, Stanford University; 2008. p. 289-302.

[352] Sensiau C, Nicoud F, Poinsot T. A tool to study azimuthal and spinning modes in annular combustors. Int J Aeroacoustics 2009;8(1):57-68.

[353] Truffin K, Poinsot T. Comparison and extension of methods for acoustic identification of burners. Combust Flame 2005;142(4):388-400.

[354] Pascaud S, Boileau M, Martinez L, Cuenot B, Poinsot T. LES of steady spray flame and ignition sequences in aeronautical combustors. In: 1st workshop INCA. SNECMA, Villaroche, France. p. 19-26. URL: http://www.cerfacs.fr/ cfdbib/repository/TR_CFD_05_70.pdf; 2005.

[355] Boudier G, Lamarque N, Staffelbach G, Gicquel L, Poinsot T. Thermo-acoustic stability of a helicopter gas turbine combustor using large-eddy simulations. Int J Aeroacoustics 2009;8(1):69-94.
[356] Staffelbach G, Gicquel L, Boudier G, Poinsot T. Large eddy simulation of selfexcited azimuthal modes in annular combustors. Proc Combust Inst 2009; 32:2909-16.

[357] Wolf P, Staffelbach G, Roux A, Gicquel L, Poinsot T, Moureau V. Massively parallel les of azimuthal thermo-acoustic instabilities in annular gas turbines. C R Acad Sci Mécanique 2009;337(6-7):385-94.

[358] Chapuis M, Fureby C, Fedina E, Alin N, Tegnér J. Les modeling of combustion applications using OpenFOAM. In: Pereira J, Sequeira A, editors. V European conference on computational dynamics. ECCOMAS CFD; 2010. p. 1-20.

[359] Brinkman W. The opportunities and challenges of exascale computing. Summary Report of the Advanced Scientific Computing Advisory Committee (ASCAC) Subcommittee; US Department of Energy, Office of Science, 2010.

[360] Boudier G, Staffelbach G, Gicquel L, Poinsot T. Mesh dependency of turbulent reacting large-eddy simulations in a gas turbine combustion chamber. In: ERCOFTAC 24-26 October, L.B., editor. QLES (Quality and reliability of LES) workshop, 2007.

[361] Jiménez C, Cuenot B, Poinsot T, Haworth D. Numerical simulation and modeling for lean stratified propane-air flames. Combust Flame 2002; 128(1-2):1-21.

[362] Senoner JM, Sanjosé M, Lederlin T, Jaegle F, Riber E, Cuenot B, et al. Evaluation of numerical strategies for LES of two-phase reacting flows. In: 2nd colloque INCA. Rouen, France: CORIA; 2008

[363] Senoner J, Sanjosé M, Lederlin T, Jaegle F, García M, Riber E, et al. Evaluation of numerical strategies for two-phase reacting flows. C R Acad Sci Mécanique 2009;337(6-7):528-38.

[364] Lecourd R, Linassier G, Lavergne G. Detailed characterisation of a swirled air kerosene spray in reactive and non reactive conditions downstream from an actual turbojet injection system. ASME2011-45173. Vancouver, Canada: ASME; 2011. Turbo Expo 2011.

[365] Dudebout $R$, James $S$. Single annular combustor for emissions reduction: technology development for small engines. In: Proceedings of 2011 ISABE conference. Stockholm, Sweden: ISABE; 2011. ISABE-2011-1119.

[366] James S. Private Communication, 2011.

[367] Eggels R. Private Communication, 2012.

[368] Wlaker D. Private Communication, 2012

[369] Baya Toda H, Turffin K, Nicoud F. Is the dynamic procedure appropriate for all sgs models? In: Pereira J, Sequeira A, editors. V European conference on computational dynamics. Lisbon, Portugal: ECCOMAS; 2010.

[370] Pierce CD, Moin P. Progress-variable approach for large eddy simulation of non-premixed turbulent combustion. J Fluid Mech 2004;504:73-97.

[371] Raman V, Pitsch H. Large eddy simulation of bluff body stabilized nonpremixed flame using a recursive-refinement procedure. Combust Flame 2005;142:329-47.

[372] Dinesh KKJR, Malalasekera WMG, Ibrahim SS, Kirkpatrick MP. Large eddy simulation of turbulent non-premixed swirling flames. ICHMT 2006;10: $593-6$.

[373] Stein O, Kempf A. Les of the Sydney swirl flame series: a study of vortex breakdown in isothermal and reacting flows. Proc Combust Inst 2007;31: 1755-63.

[374] Kempf A, Malalasekera W, Rang Dinesh KKJ, Stein O. Large eddy simulations of swirling non-premixed flames with flamelet models: a comparison of numerical methods. Flow Turb Combust 2008;81(4):523-61.

[375] Malalasekera W, Rang Dinesh KKJ, Ibrahim SS, Masri AR. Les of recirculation and vortex breakdown in swirling flames. Combust Sci Technol 2008;180(5): 809-32.

[376] El-Asrag H, Menon S. Large eddy simulation of bluff-body stabilized swirling non-premixed flames. Proc Combust Inst 2007;31:1747-54

[377] James S, Zhu J, Anand M. Les of turbulent flames using the filtered density function model. Proc Combust Inst 2007;31:1737-45.

[378] Domingo P, Vervisch L, Veynante D. Large-eddy simulation of a lifted methane jet flame in a vitiated coflow. Combust Flame 2008;152(3):415-32.

[379] Nogenmyr KJ, Fureby C, Bai X, Petersson P, Collin R, Linne M. Large eddy simulation and laser diagnostic studies on a low swirl stratified premixed flame. Combust Flame 2009;156:25-36.

[380] Grinstein F, Fureby C. Les studies of the flow in a swirl gas combustor. Proc Combust Inst 2005;30(2):1791-8.

[381] Kim WW, Menon S, Mongia HC. Large-eddy simulation of a gas turbine combustor. Combust Sci Technol 1999;143(1-6):25-62.

[382] Kim WW, Menon S. An unsteady incompressible navier stokes solver for LES of turbulent flows. Int J Numer Meth Fluids 1999;31:983-1017.

[383] Fureby C. A fractal flame-wrinkling large-eddy simulation model for premixed turbulent combustion. Proc Combust Inst 2005;30(1):593-601.

[384] Duwig C, Fuchs L. Study of flame stabilization in a swirling combustor using a new flamelet formulation. Combust Sci Technol 2005;177(8):1485-510.

[385] Shinjo J, Matsuyama S, Mizobuchi Y, Ogawa S. Study on flame dynamics with secondary fuel injection control by large eddy simulation. Combust Flame 2007;150(4):277-91.

[386] Selle L, Benoit L, Poinsot T, Nicoud F, Krebs W. Joint use of compressible large-eddy simulation and helmoltz solvers for the analysis of rotating modes in an industrial swirled burner. Combust Flame 2006;145(1-2): 194-205.

[387] Sommerer Y, Galley D, Poinsot T, Ducruix S, Lacas F, Veynante D. Large eddy simulation and experimental study of flashback and blow-off in a lean partially premixed swirled burner. J Turb 2004;5. 
[388] Huang Y, Sung HG, Hsieh SY, Yang V. Large eddy simulation of combustion dynamics of lean-premixed swirl-stabilized combustor. J Prop Power 2003; 19(5):782-94.

[389] Huang Y, Yang V. Bifurcation of flame structure in a lean premixed swirlstabilized combustor: transition from stable to unstable flame. Combust Flame 2004;136:383-9.

[390] Huang Y, Yang V. Effect of swirl on combustion dynamics in a lean-premixed swirl-stabilized combustor. Proc Combust Inst 2005;30:1775-82.

[391] Huang Y, Wang S, Yang V. Systematic analysis of lean-premixed swirlstabilized combustion. Am Inst Aeronaut Astronaut J 2006;44(724-740)

[392] Sankaran V, Menon S. Les of spray combustion in swirling flows. J Turb 2002; 3:011.

[393] Cannon S, Adumitroaie V, Smith C. Les modeling of combustion dynamics in lean-premixed combustors. 2001-GT-375. ASME; 2001.

[394] Cannon S, Zuo B, Smith C. Les predictions of combustor emissions from a practical industrial fuel injector. 203-GT-38200. ASME; 2003.

[395] Stone C, Menon S. Swirl control of combustion instabilities in a gas turbine combustor. Proc Combust Inst 2002;29(1):155-60.

[396] Stone C, Menon S. Open-loop control of combustion instabilities in a model gas turbine combustor. J Turb 2003;4:020.

[397] Pitsch H, de la Geneste LD. Large eddy simulation of premixed turbulent combustion using a level-set approach. Proc Combust Inst 2002:29:2001-8.

[398] Eggenspieler G, Menon S. Modeling pollutant formation near lean blow-out in gas turbine engines. In: Recent advances in direct and large-eddy simulations. Boston: Kluwer Academic; 2003. p. 351-8.

[399] Eggenspieler G, Menon S. Large-eddy simulation of pollutant emission in a doe-hat combustor. J Prop Power 2004;20(6):1076-85.
[400] Menon S, Patel N. Subgrid modeling for simulation of spray combustion in large scale combustors. Am Inst Aeronaut Astronaut J 2006;44(4):709-23.

[401] Patel N Menon S. Simulation of spray-turbulence-flame interactions in a lean direct injection combustor. Combust Flame 2008;153(1-2):228-57.

[402] Schmitt P, Poinsot T, Schuermans B, Geigle KP. Large-eddy simulation and experimental study of heat transfer, nitric oxide emissions and combustion instability in a swirled turbulent high-pressure burner. J Fluid Mech 2007; 570:17-46. http://dx.doi.org/10.1017/S0022112006003156.

[403] Bicen AF, Tse DGN, Whitelaw JH. Combustor, characteristics of a model cantype combustor. Tech. Rep. Rep. FS/87/28. London: Imperial College; 1987.

[404] Bicen AF, Tse DGN, Whitelaw JH. Combustion characteristics of a model cantype combustor. Combust Flame 1990;80(2):111-25.

[405] Staffelbach G, Poinsot T. High performance computing for combustion applications. In: Super computing 2006; 2006. Tampa, Florida, USA.

[406] Moin P. Large eddy simulation of multi-phase turbulent flows in realistic combustors. Prog Comput Fluid Dynam 2004;4:237-40.

[407] Li S, Williams F, Gebert K. A simplified, fundamentally based method for calculating nox emissions in lean premixed combustors. Combust Flame 1999;119:367-73.

[408] Mongia H. Taps - a 4th generation propulsion combustor technology for low emissions. In: The next 100 years, international air \& space symposium and exposition. Dayton, USA: AIAA Paper 2003-2657; 2003.

[409] Richardson S, Mastorakos E. Conditional moment closure modelling for spark ignition in a turbulent n-heptane spray. In: 5 th Mediterranean combustion symposium; 2007. Monastir, Tunisia.

[410] Batchelor GK. The role of big eddies in homogeneous turbulence. Proc Roy Soc A: Math, Phys Eng Sci 1949;195(1043):513-32. 